\title{
Efficient Base-Free Aqueous Reforming of Methanol Homogeneously Catalyzed by Ruthenium Exhibiting a Remarkable Acceleration by Added Catalytic Thiol
}

Jie Luo, Sayan Kar, Michael Rauch, Michael Montag, Yehoshoa Ben-David, David Milstein*

Department of Molecular Chemistry and Materials Science, Weizmann Institute of Science, Rehovot, 76100, Israel

E-mail: david.milstein@weizmann.ac.il

Table of Contents

Supporting Notes

Supporting Experimental Procedures

1. General considerations

2. Preparation of standard curves for $\mathrm{CO}_{2}, \mathrm{H}_{2}$ and $\mathrm{CO}$

3. General experimental procedures

4. Representative GC spectra

5. Condition screening

6. Control experiments

7. Computational details 


\section{Supporting Notes}

Note S1. Proposed pathways and potential energy surface

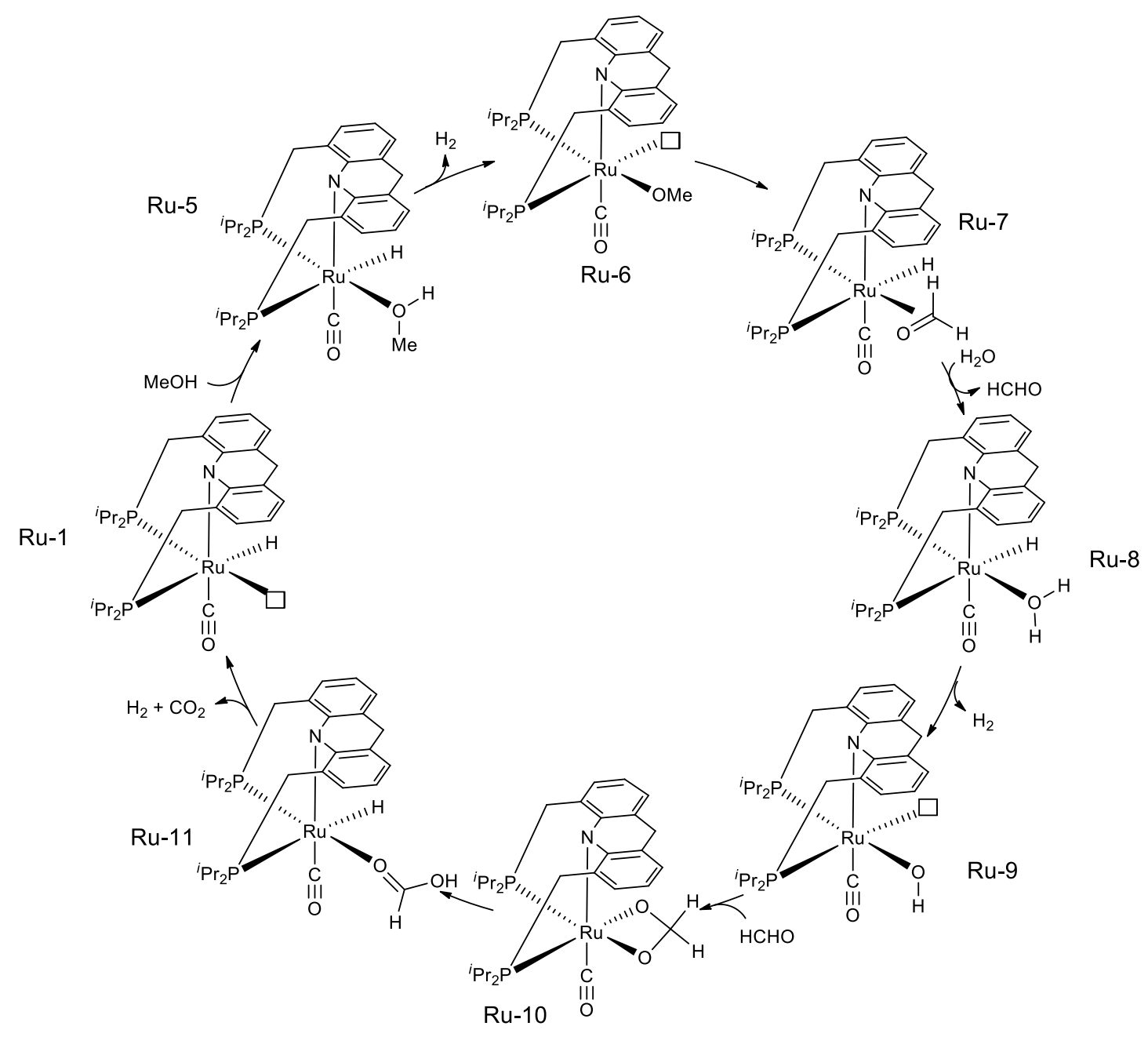

Figure S1. Proposed mechanism including inner-sphere dehydrogenation of water. 


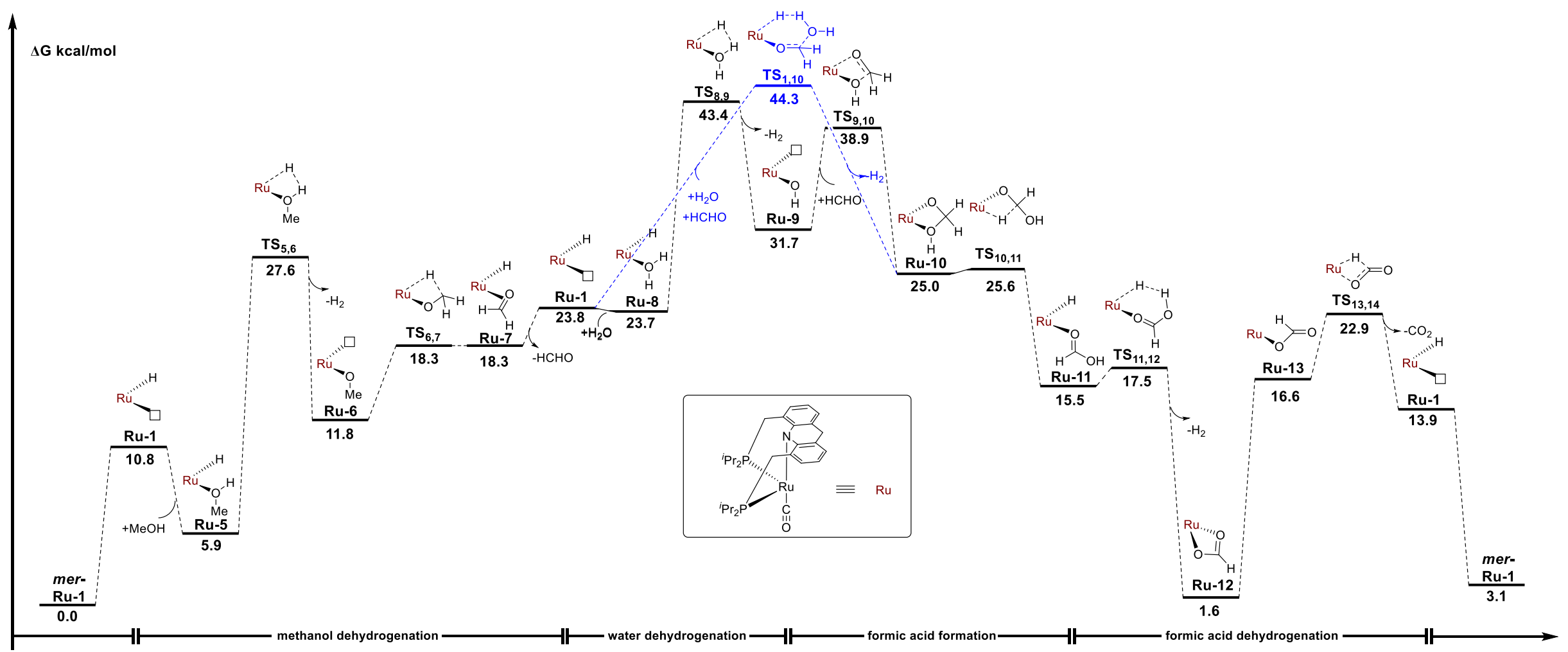

Figure S2. Potential energy surface for the pathway including inner-sphere dehydrogenation of water (in $\mathrm{MeOH}, \mathrm{T}=383.15 \mathrm{~K}$, pressure $=1$ ). Note that Ru-7 with formaldehyde coordinated in a $\kappa^{1}-\mathrm{O}$ fashion is $6.0 \mathrm{kcal} / \mathrm{mol}$ higher in energy than $\mathbf{R u}-\mathbf{7}$ with $\eta^{2}$-coordinated $\mathrm{C}=\mathrm{O}$, which is presented in this Figure. 


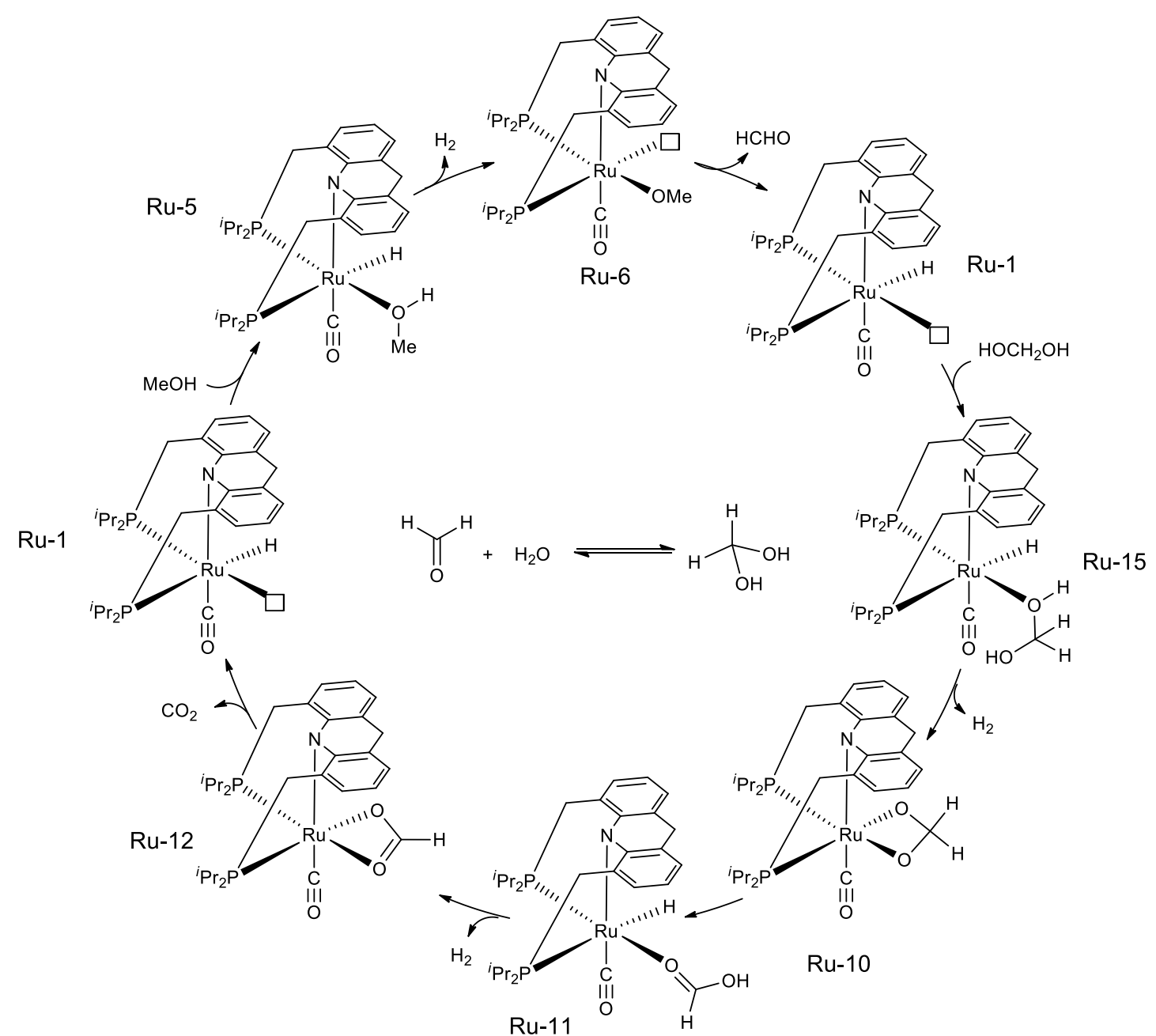

Figure S3. Proposed mechanism including inner-sphere dehydrogenation of methandiol. 


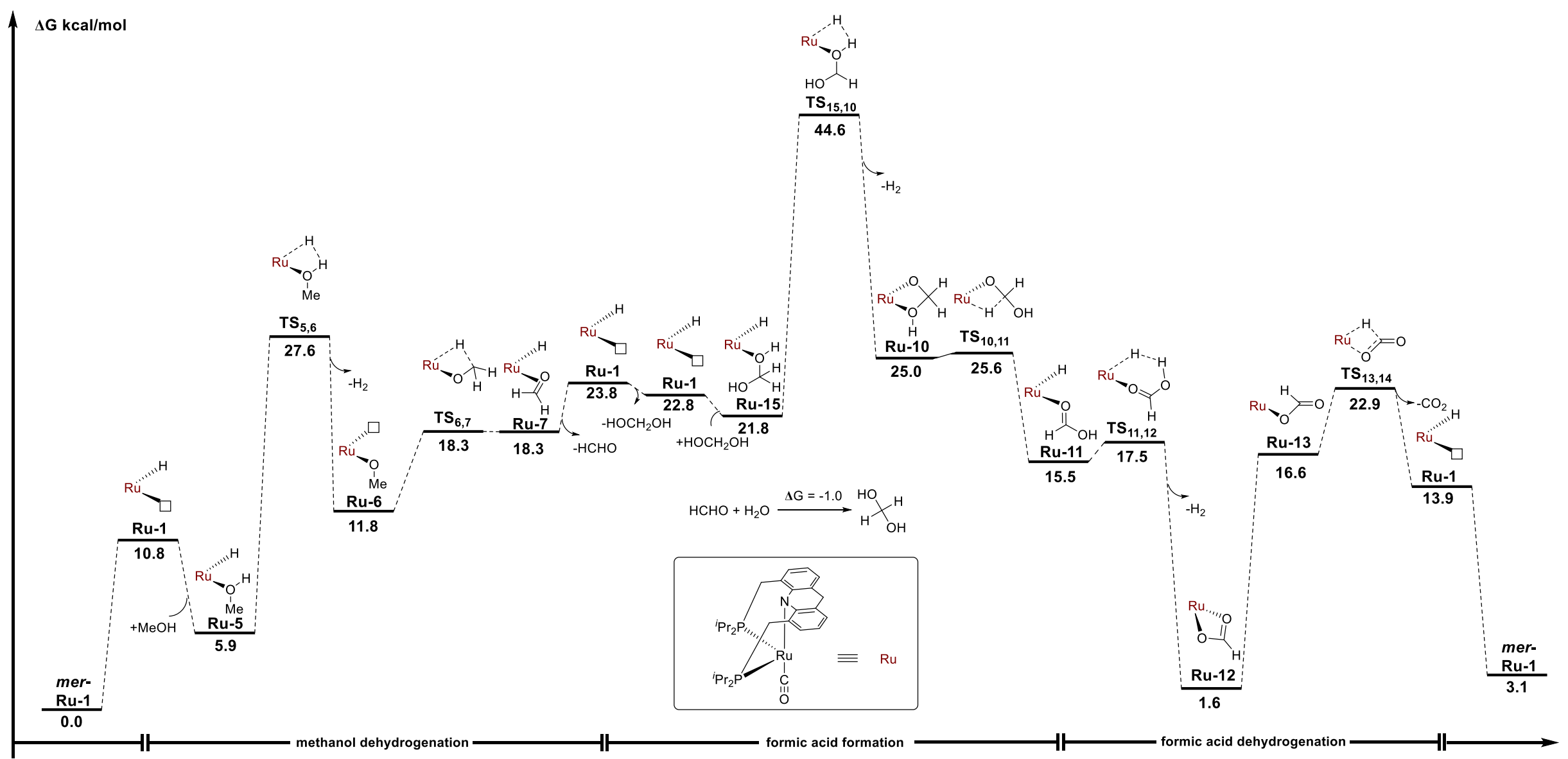

Figure S4. Potential energy surface for the pathway including inner-sphere dehydrogenation of methandiol (in $\mathrm{MeOH}, \mathrm{T}=383.15 \mathrm{~K}$, pressure $=$ $1)$. 


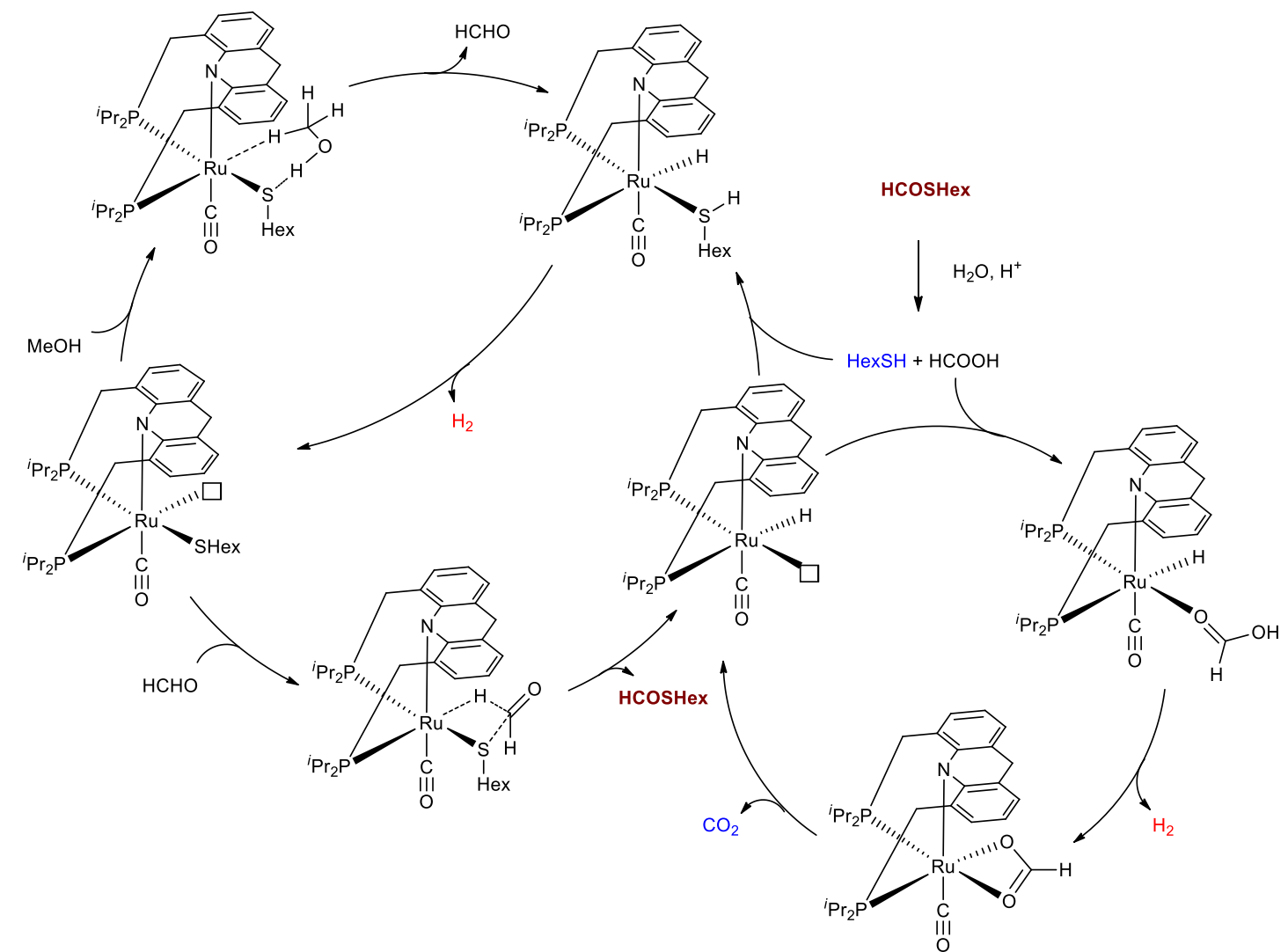

Figure S5. Proposed mechanism through thioester formation, excluded by experimental results. 


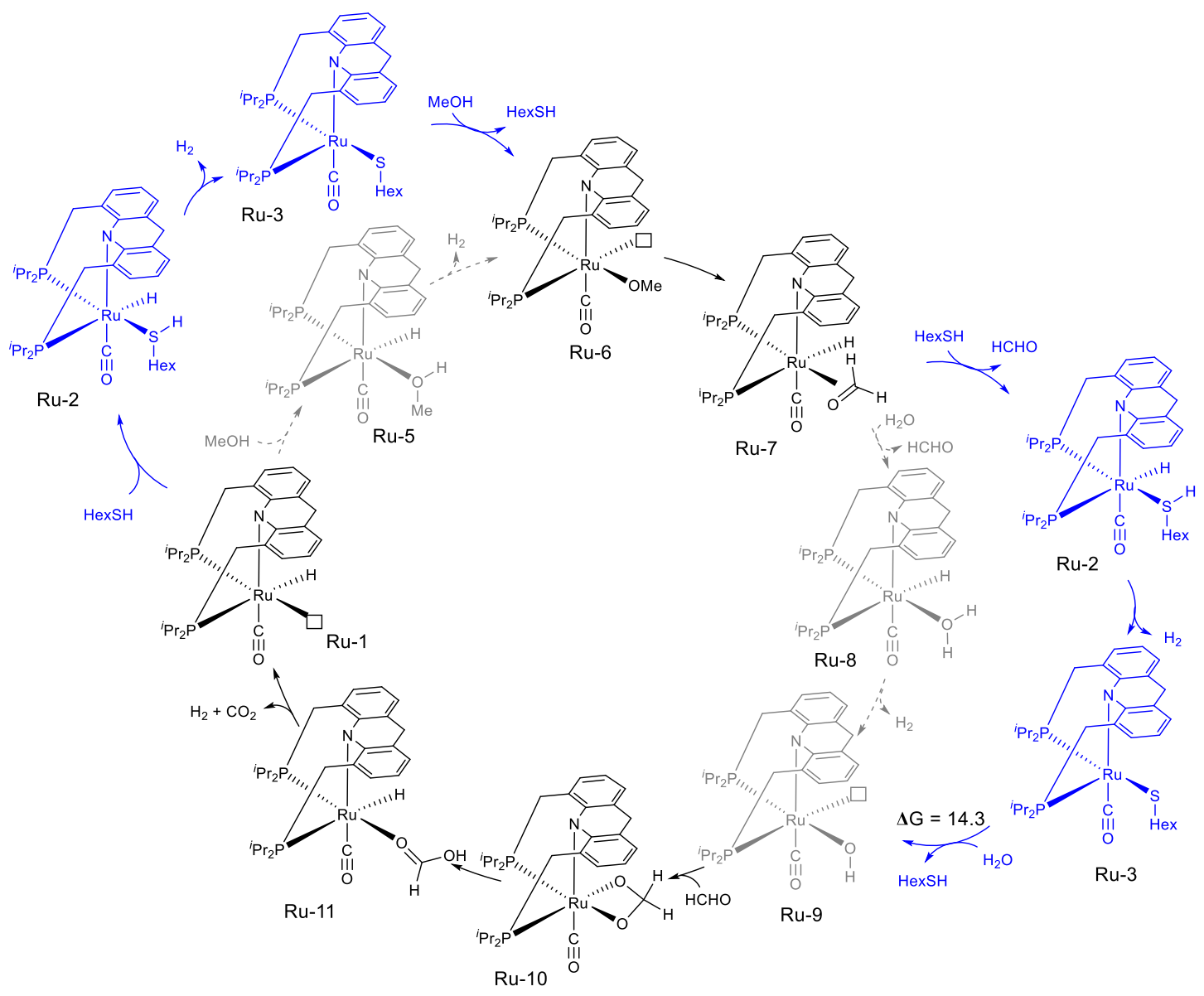

Figure S6. Proposed mechanism via exchange of thiol. 


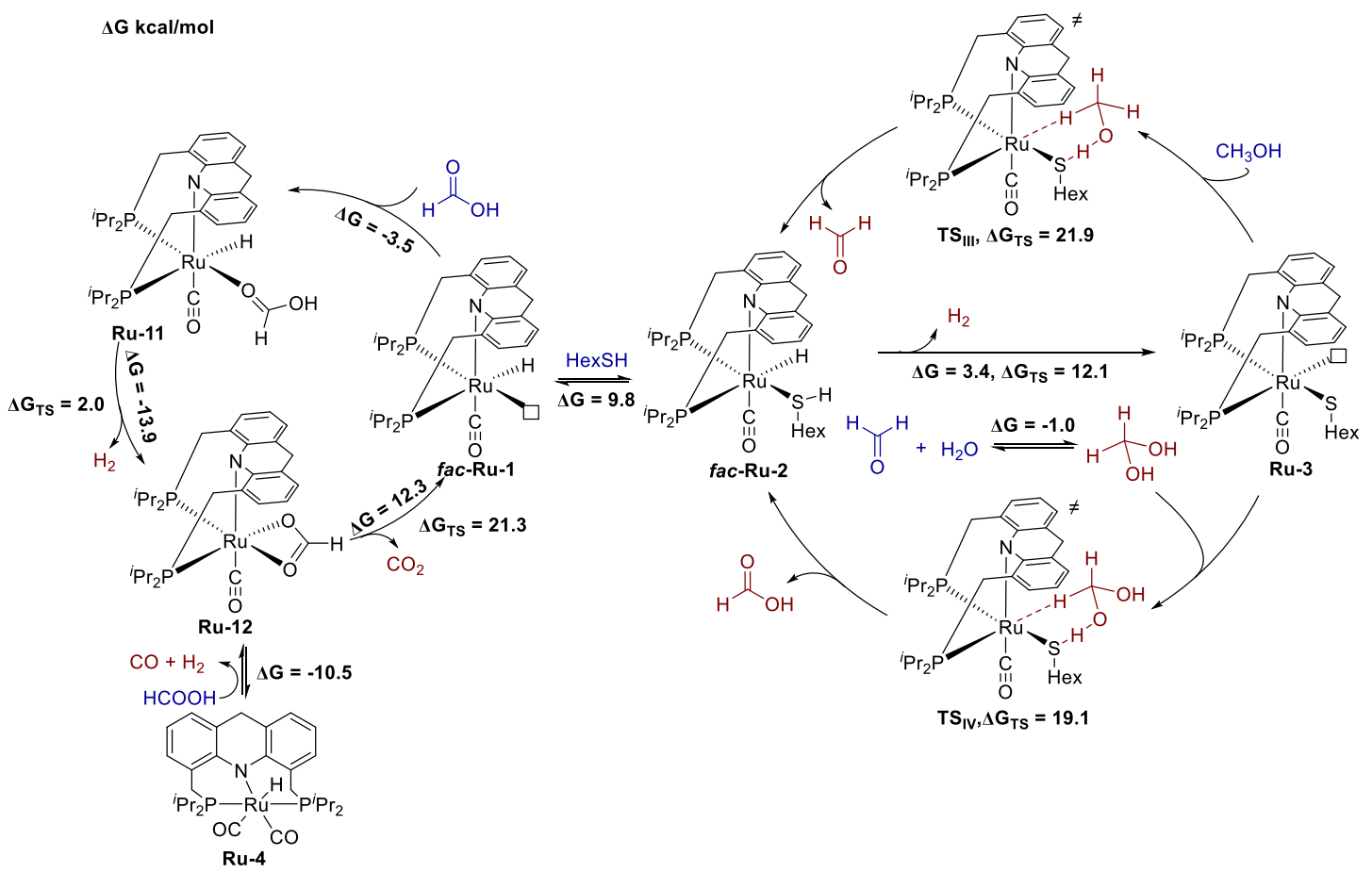

Figure S7. Proposed mechanism with calculated Gibbs energy difference between each intermediates and transition states based on outer-sphere dehydrogenation of methanol and methandiol involving Ru-4; The transformations of hydrogen, $\mathrm{CO}_{2}$ and $\mathrm{CO}$ from the condensed phase to the gas phase are not additionally corrected in the free energy quantities provided. 


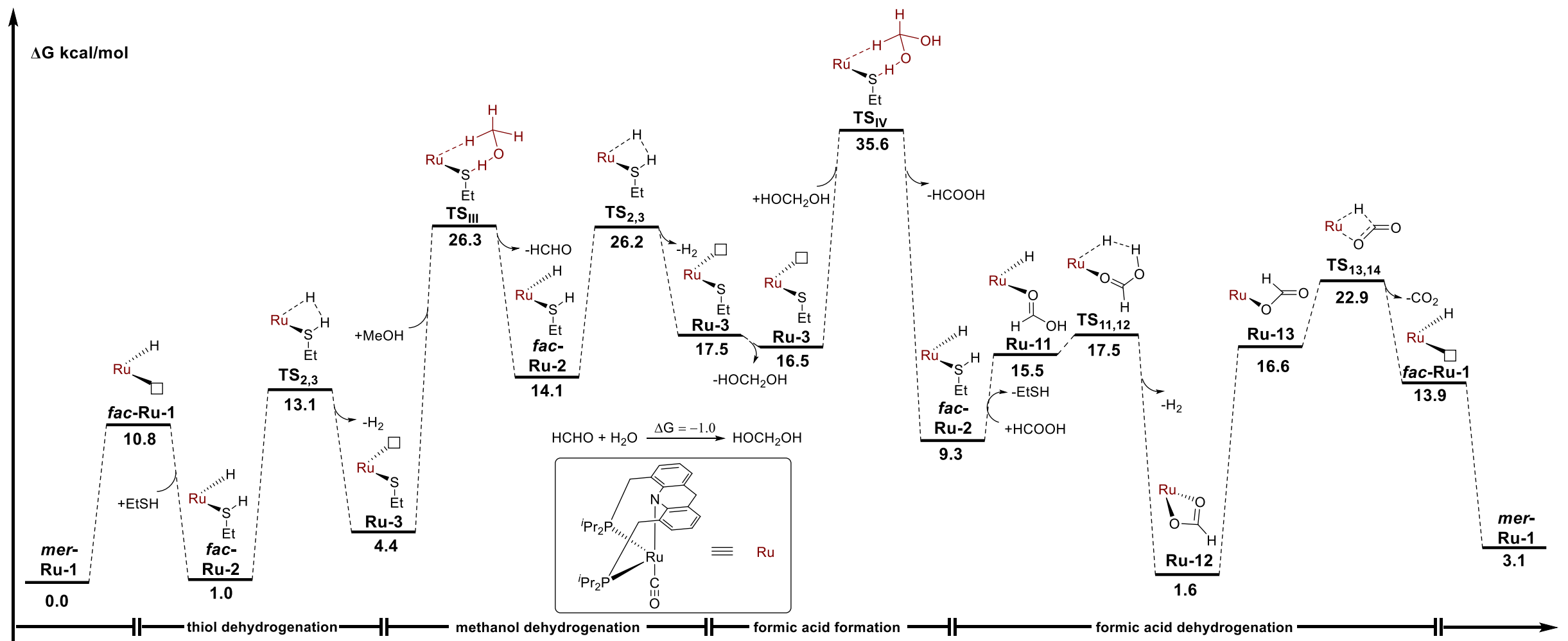

Figure S8. Potential energy surface for the pathway including outer-sphere dehydrogenation of methanol and methandiol based on ruthenium thiolate complex (in $\mathrm{MeOH}, \mathrm{T}=383.15 \mathrm{~K}$, pressure $=1$ ). 


\section{Note S2. Control experiments with $\mathrm{Ru}-4$}

Control experiments were carried out using Ru-4 as the catalyst. Interestingly, under the reaction conditions with the addition of 1 equiv thiol, $240 \mathrm{~mL}$ gas was collected after $12 \mathrm{~h}$ heating at $150{ }^{\circ} \mathrm{C}$ (Supporting Figure 9). However, nearly no gas was collected without the addition of thiol.
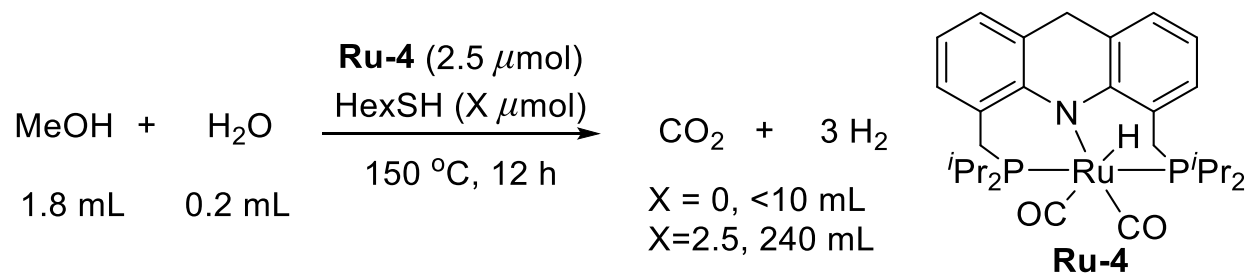

Figure S9. Control experiments based on Ru-4.

To figure out how Ru-4 worked in the reaction system in the presence of thiol, mechanistic studies were conducted heating the biscarbonyl acridine Ru-complex Ru-4 in acetic acid under Ar flow. The utilization of acetic acid is to mimic formic acid in the methanol reforming system, which under the reaction conditions is quite easy to undergo dehydrogenation and thus makes the capture of the key intermediate, ruthenium formate, very difficult. Interestingly, both $\mathrm{CO}$ and $\mathrm{H}_{2}$ were detected by GC during the reaction and the ruthenium acetate complex Ru-16 was generated as the major product (Figure S10). ${ }^{1}$ The result proves the lability of the second $\mathrm{CO}$ on the ruthenium center and thus in the case of current methanol reforming reaction, it is reasonable to propose that $\mathbf{R u}-\mathbf{4}$ can also react with the intermediate formic acid to release one molecule of $\mathrm{CO}$ and $\mathrm{H}_{2}$ to regenerate monocarbonyl complex Ru-12. The Gibbs energy difference between ruthenium formate complex $\mathbf{R u - 1 2}$ and $\mathbf{R u - 4}$ is calculated as $+10.5 \mathrm{kcal} / \mathrm{mol}$ (from Ru-12 to Ru-4, Supporting Figure 7), which can be overcome under the reaction conditions. It is noted the transformation of hydrogen and $\mathrm{CO}$ from the condensed phase to the gas phase is not additionally corrected in the free energy quantities. ${ }^{2}$

Based on the above results, it is proposed that $\mathbf{R u}-\mathbf{4}$ is the off-cycle resting state of the catalyst during most of the reaction time. Even though Ru-4 might be able to catalyze the reaction, it should be very slow as reflected from ref. 1 by the calculated 
activation barriers for the dehydrogenation of formic acid (overall kinetic barrier of $41.9 \mathrm{kcal} / \mathrm{mol}$ with Ru-4 vs $25.1 \mathrm{kcal} / \mathrm{mol}$ with Ru-1 in benzene). Thus in the current methanol reforming system, the monocarbonyl catalysts $\mathbf{R u - 1}-\mathbf{R u - 3}$ are likely still the actual catalysts of the reaction despite the generation of $\mathrm{CO}$.
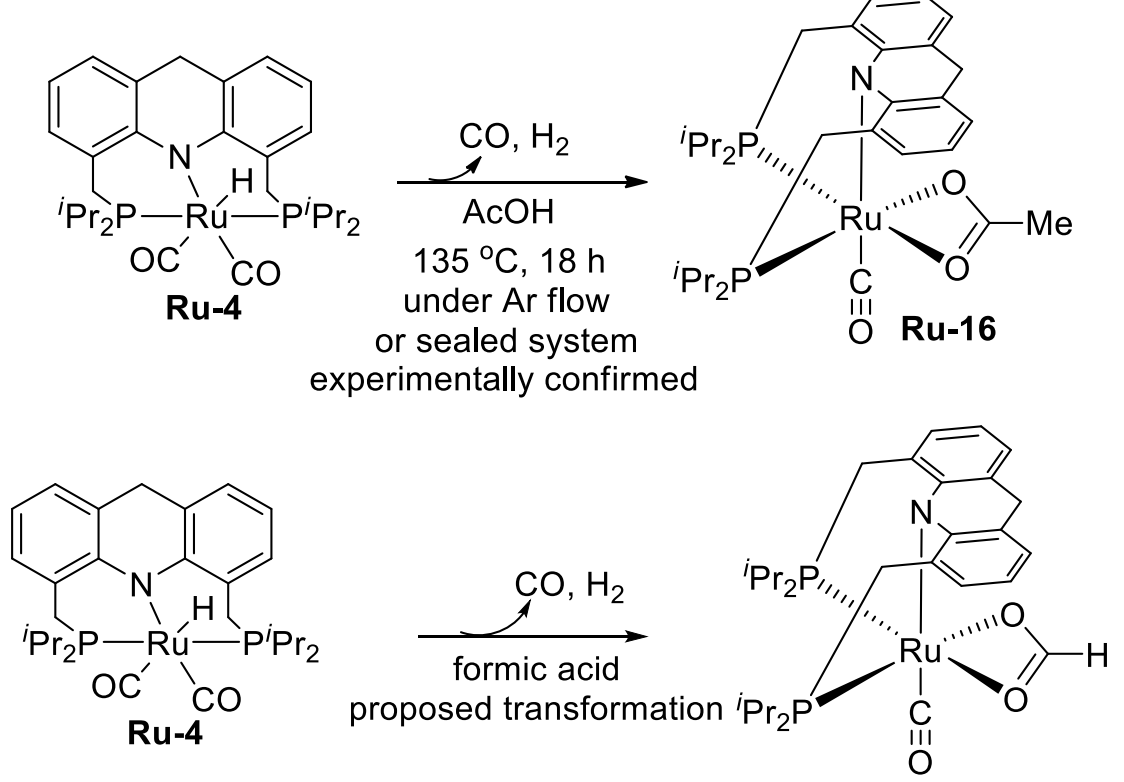

Ru-12

Figure S10. Control experiments relevant to CO loss from Ru-4. 


\section{Note S3. Factors related to the amount of $\mathrm{CO}$ generated in the reaction}

1. Reaction temperature. Control experiments were carried out heating the reaction at different temperature. While nearly no $\mathrm{CO}$ was detected in the collected gas after heating at $120{ }^{\circ} \mathrm{C}$, higher concentration of $\mathrm{CO}$ was observed after heating at $135{ }^{\circ} \mathrm{C}$ and $150{ }^{\circ} \mathrm{C}$ (Supporting Figure S11). Thus, the reaction temperature can affect the generation of $\mathrm{CO}$ in the system. ${ }^{3-6}$ One thing worthwhile to mention is the internal temperature of the system. Since we employed a closed system, the internal temperature of the reaction increased along with the accumulation of the pressure, which might also affect the generation of $\mathrm{CO}$ at different stage of the reaction.

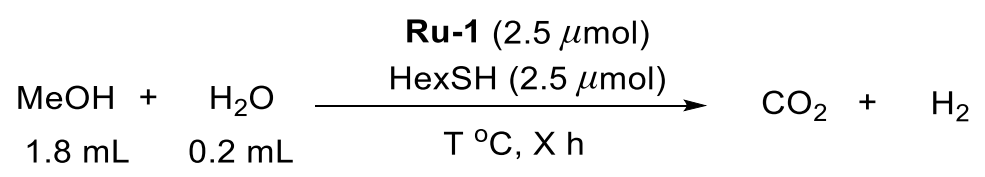

\begin{tabular}{|c|c|c|c|}
\hline $120^{\circ} \mathrm{C}, 36 \mathrm{~h}$ & $135^{\circ} \mathrm{C}, 24 \mathrm{~h}$ & $150^{\circ} \mathrm{C}, 12 \mathrm{~h}$ & $150^{\circ} \mathrm{C}, 1 \mathrm{~h}$ \\
\hline $\begin{aligned} & 110 \mathrm{~mL} \\
< & 20 \mathrm{ppm} \mathrm{CO}\end{aligned}$ & $\begin{array}{c}150 \mathrm{~mL} \\
154 \mathrm{ppm} \mathrm{CO}\end{array}$ & $\begin{array}{c}470 \mathrm{~mL} \\
181 \mathrm{ppm} \mathrm{CO}\end{array}$ & is bubblec \\
\hline TOF $\left(\mathrm{H}_{2}\right): 39 \mathrm{~h}^{-1}$ & TOF $\left(\mathrm{H}_{2}\right): 76 \mathrm{~h}^{-1}$ & TOF $\left(\mathrm{H}_{2}\right): 480 \mathrm{~h}^{-1}$ & \\
\hline
\end{tabular}

Figure S11. Reaction temperature screening.

2. Generation of Ru-4. The employed catalyst Ru-1 can absorb a little amount of CO to generate a biscarbonyl ruthenium complex Ru-4 (Supporting Figure S12), ${ }^{7}$ which is supposed to lower the amount of $\mathrm{CO}$ in the collected gas. Note that in addition to the direct coordination by CO, Ru-4 can also be generated by the reaction of $\mathbf{R u - 1}$ either with formic acid (Supporting Table S1), or with $\mathrm{CO}_{2}$ and $\mathrm{H}_{2}$ (Supporting Figure S35).

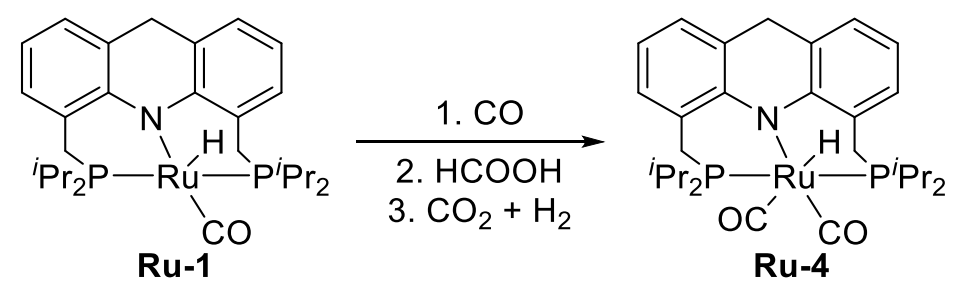

Figure S12. Generation of Ru-4 from Ru-1 with CO. 
3. Concentration of water in the system. Comparing to the result from $9: 1$ $\mathrm{MeOH}: \mathrm{H}_{2} \mathrm{O}$ system, the amount of $\mathrm{CO}$ was detected in a very low level in MeOH: $\mathrm{H}_{2} \mathrm{O}=4: 1$ or $6: 1$ system after $12 \mathrm{~h}$ heating, which possibly indicated that the concentration of water in the system might affect the generation of CO (Supporting Figure S13).

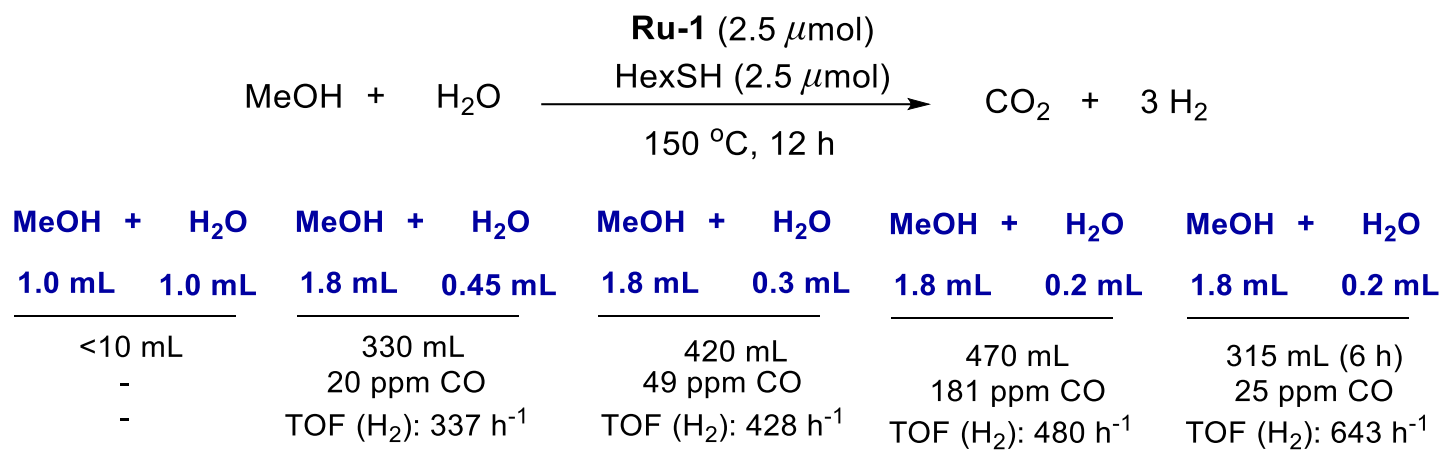

Figure S13. Detailed results of solvent optimization.

In the first $72 \mathrm{~h}$ of the long-term reaction (in $3.6 \mathrm{~mL} \mathrm{MeOH}$ and $0.4 \mathrm{~mL} \mathrm{H}_{2} \mathrm{O}$ ), the amount of $\mathrm{CO}$ increased as the reaction continued. For example, while only $0.02 \%$ $\mathrm{CO}$ was detected in the collected gas of the first cycle (first $12 \mathrm{~h}$ ), higher amounts of $\mathrm{CO}$ were detected in the following 5 cycles (Supporting Figure S14). Considering water was consumed during the reaction, making its concentration continuously decreased, these results also supported the concentration of water in the system could affect the generation of $\mathrm{CO}$.

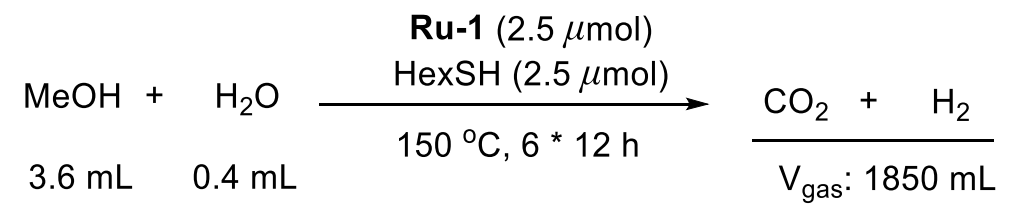

\begin{tabular}{|c|c|c|c|c|c|c|}
\hline Cycle/12 h & 1st & 2nd & 3rd & 4th & 5th & 6th \\
\hline V gas $(\mathrm{mL})$ & 460 & 265 & 360 & 220 & 265 & 280 \\
\hline CO $(\mathrm{ppm})$ & 196 & 239 & 391 & 357 & 442 & 681 \\
\hline
\end{tabular}

Figure S14. Increasing amount of CO during the reaction. 


\section{Note S4. Control experiments on the source of $\mathrm{CO}$}

1. Reverse water-gas-shift reaction. The reverse water-gas-shift reaction ${ }^{8,9}$ was tested (See Supporting Figure S15), however, no CO was detected in the resulting gas mixture. The same result was obtained directly starting with Ru-1 as the catalyst without the addition of a catalytic amount of thiol (See Supporting Figure S35).

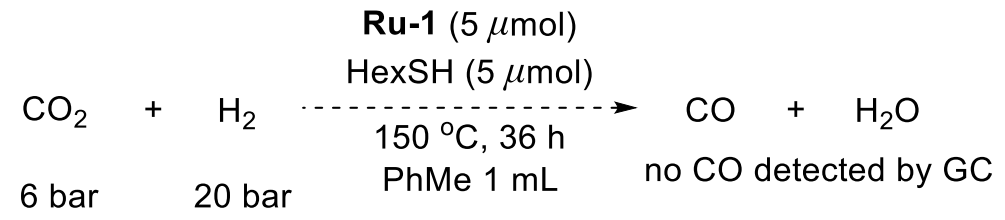

Figure S15. Test the possibility of reverse water-shift reaction.

2. Thermo-decomposition of formic acid. ${ }^{6}$ The dehydrogenation of formic acid was examined under the reaction conditions with or without water (Supporting Table S1). As expected, with Ru-1 as the catalyst, the reactions were very efficient heating at $150{ }^{\circ} \mathrm{C}$ with a TOF $\left(\mathrm{H}_{2}\right)$ more than $10,000 \mathrm{~h}^{-1}$ in 10 minutes. However, no $\mathrm{CO}$ was detected after the reaction in the collected gas (entries 1 and 2). Similar results were obtained employing Ru-2 as the catalyst with only trace amount of CO detected in the collected gas (entries 3 and 4, Ru-2 was prepared in situ). The efficiency of Ru-2 is slightly lower than that of Ru-1, which also suggests that Ru-1 is the real catalyst for the dehydrogenation of formic acid step in current methanol reforming reaction. ${ }^{1}$ In addition, the biscarbonyl ruthenium complex Ru-4 was also used as the catalyst (entries 5 and 6). Although the reaction rate decreased, still no $\mathrm{CO}$ was detected in the collected gas after 20 min heating.

Table S1. Dehydrogenation of formic acid

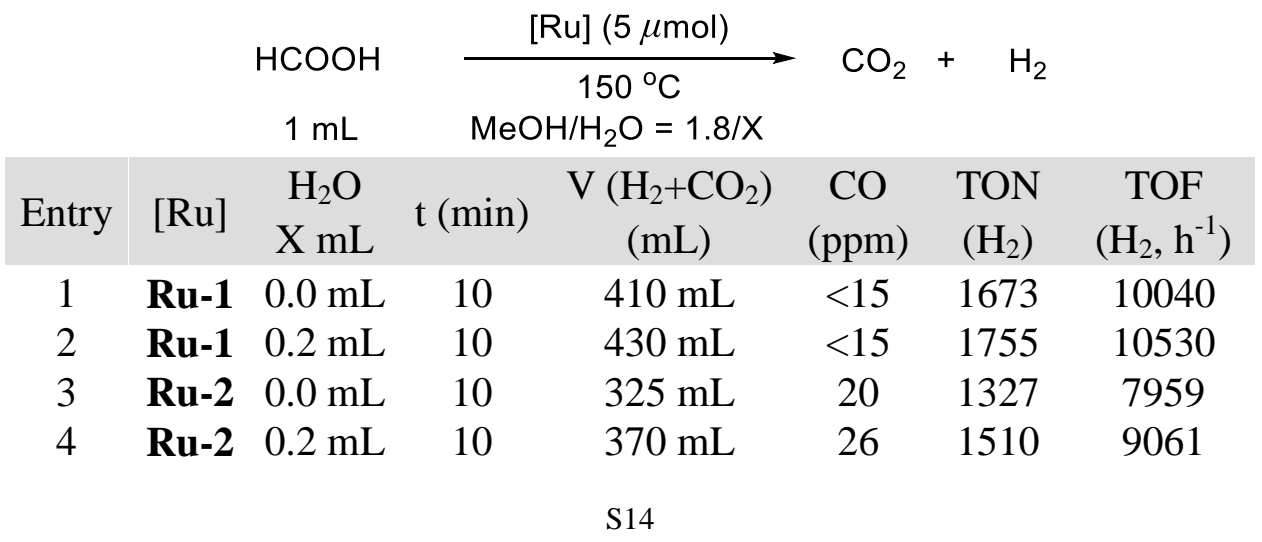


$\begin{array}{llllllll}5 & \mathbf{R u}-4 & 0.0 \mathrm{~mL} & 20 & 300 \mathrm{~mL} & <15 & 1224 & 3673\end{array}$

$6 \quad$ Ru-4 $\quad 0.2 \mathrm{~mL} \quad 20 \quad 335 \mathrm{~mL} \quad<15 \quad 1367 \quad 4102$

Conditions: [Ru] $(5 \mu \mathrm{mol}), \mathrm{MeOH} / \mathrm{H}_{2} \mathrm{O}$ as indicated, heat in a closed $90 \mathrm{~mL}$ Fischer-Porter tube in a $150{ }^{\circ} \mathrm{C}$ oil bath; Gas composition of $\sim 1: 1 \mathrm{CO}_{2}: \mathrm{H}_{2}$ for each case. Detection limit of the GC $(<15 \mathrm{ppm})$; In entries $1-4$, the resulting ruthenium species after heating is Ru-4.

3. Thermo-decomposition of formaldehyde. ${ }^{3-5}$ Trioxane (anhydrous formaldehyde surrogate) was tested under the reaction conditions. In order to avoid its further conversion into formic acid, $\mathrm{MeOH}$ was used as the only solvent without the addition of water. Moreover, in consideration of internal temperature and atmosphere in the real system, 4 bar $\mathrm{H}_{2}$ gas was introduced before the reaction in the control experiments (Figure S16). Interestingly, after $24 \mathrm{~h}$ heating, $0.28 \% \mathrm{CO}$ was detected by $\mathrm{GC}$ in the collected gas (Note little amount of $\mathrm{CO}_{2}$ was detected by $\mathrm{GC}$, indicating little amount of water existed in the system). A similar result was also obtained using Ru-1 as the catalyst. These experiments support the generated $\mathrm{CO}$ in the developed methanol reforming system mostly comes from the thermo-decomposition of the formaldehyde intermediate.

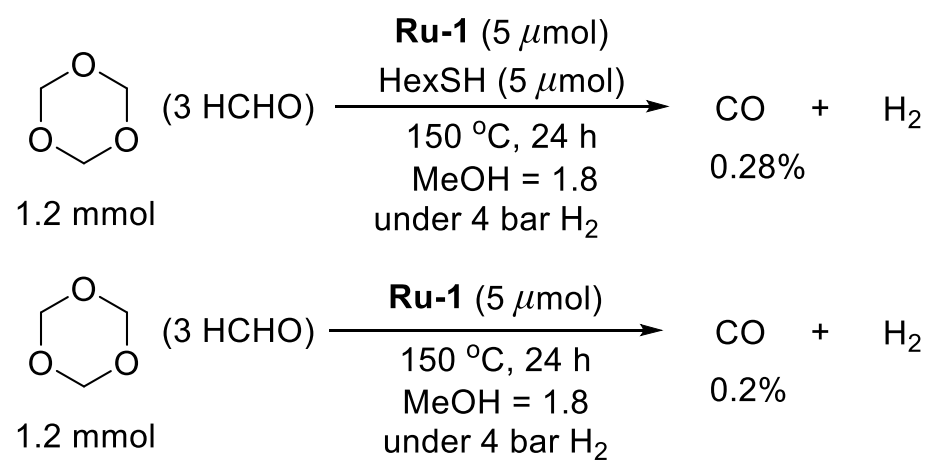

Figure S16. Decomposition of formaldehyde to $\mathrm{CO}$ and $\mathrm{H}_{2}$. 


\section{Supporting Experimental Procedures}

\section{General considerations}

All experiments with metal complexes and phosphine ligands were carried out under an atmosphere of purified nitrogen in a Vacuum Atmosphere glovebox equipped with a MO 40-2 inert gas purifier or using standard Schlenk techniques. All solvents were reagent grade or better. All non-deuterated solvents were purified according to standard procedures under argon atmosphere. Deuterated solvents were degassed with argon and directly used. All solvents were degassed with argon and kept in the glove box over $3 \AA$ molecular sieves. Water used in this system is deionized water which is further degassed with argon and kept in glovebox. All ${ }^{1} \mathrm{H}$ NMR, ${ }^{13} \mathrm{C}$ NMR or ${ }^{31} \mathrm{P}$ NMR spectra were recorded on a Bruker AVANCE III $300 \mathrm{MHz}, 400 \mathrm{MHz}$ spectrometer and reported in ppm $(\delta)$. Chemical shifts were referenced to the residual solvent peaks $\left({ }^{1} \mathrm{H} \mathrm{NMR},{ }^{13} \mathrm{C} \mathrm{NMR}\right)$ or an external standard of phosphoric acid ( $85 \%$ solution in $\left.\mathrm{D}_{2} \mathrm{O}\right)$ at $0.0 \mathrm{ppm}\left({ }^{31} \mathrm{P} \mathrm{NMR}\right)$. NMR spectroscopy abbreviations: br, broad; s, singlet; d, doublet; t, triplet; q, quartet; m, multiplet. GC analysis was performed on an HP 6890 chromatograph (TCD detector) with helium as the carrier gas.

Complexes $\mathbf{R u}-\mathbf{1}^{10}, \mathbf{R u}-\mathbf{2}^{11}, \mathbf{R u}-\mathbf{3}^{11}, \mathbf{R u}-\mathbf{4}^{7}$ were prepared according to literature procedures.

The scale of the graduated cylinder is $10 \mathrm{~mL}$ and the last digit of the volume of collected gas is estimated as 5 or 0 .

The turnover number (TON) of $\mathrm{H}_{2}$ was calculated from the amount of gas collected. $\mathrm{GC}$ analysis indicate the composition of gas is almost $3: 1 \mathrm{H}_{2} / \mathrm{CO}_{2}(<0.1 \% \mathrm{CO}$ is not taken into consideration). Every hydrogen molecule represents one catalyst turnover number. Thus, the TON $\left(\mathrm{H}_{2}\right)$ value was calculated as:

$$
\operatorname{TON}\left(\mathrm{H}_{2}\right)=\mathrm{V}_{\text {gas }} \times(3 / 4) \div 24.5 \div \mathrm{n}_{\text {cat }}
$$




\section{Preparation of standard curves for $\mathrm{CO}_{2}, \mathrm{H}_{2}$ and $\mathrm{CO}$}

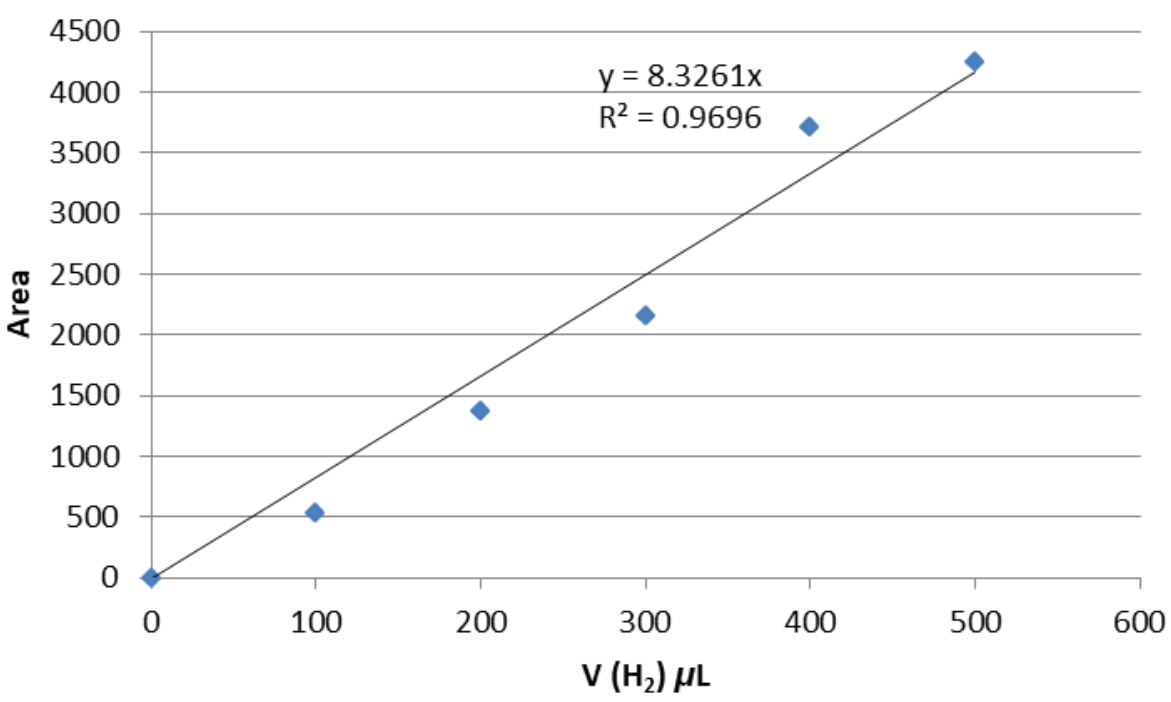

Figure S17. Standard Curve of $\mathrm{H}_{2}$ in GC.

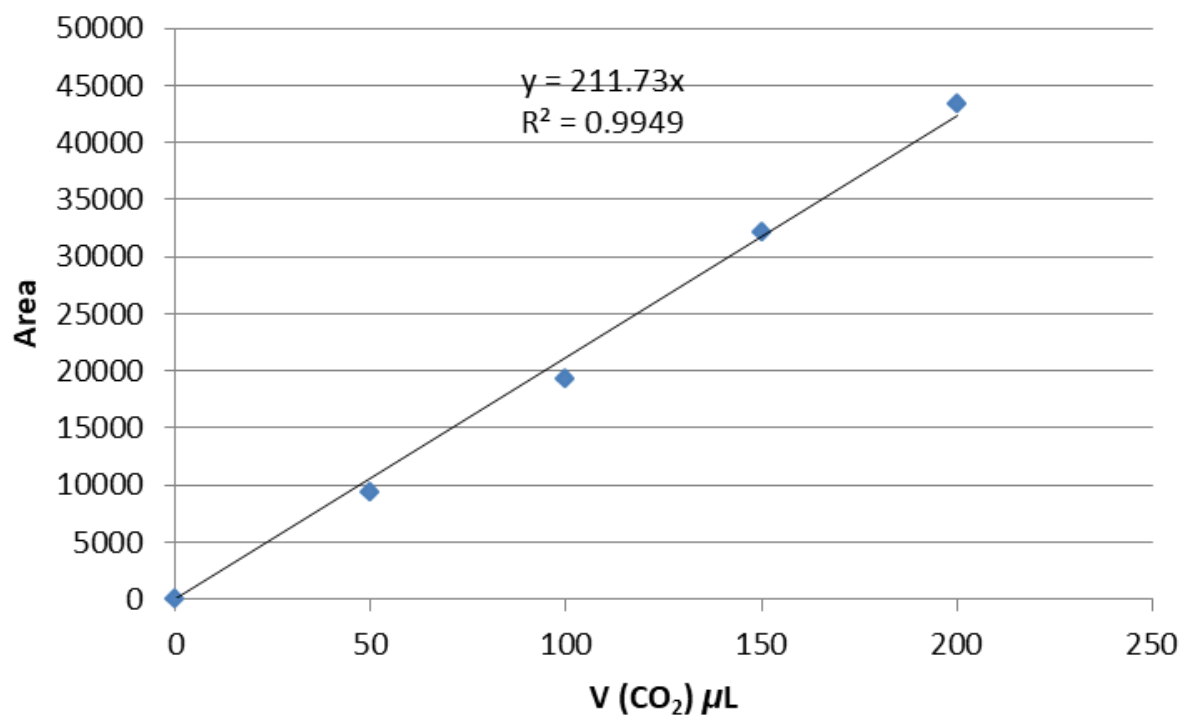

Figure S18. Standard Curve of $\mathrm{CO}_{2}$ in $\mathrm{GC}$. 


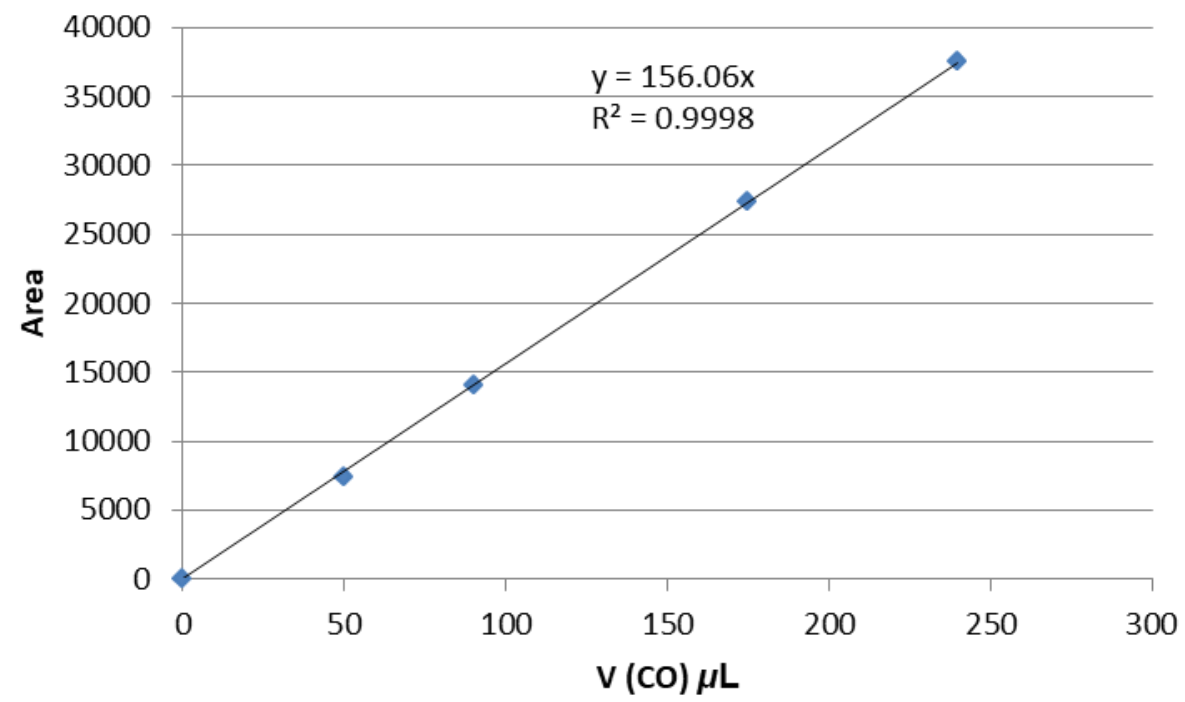

Figure S19. Standard Curve of CO in GC. 


\section{General experimental procedures}

$$
\mathrm{CH}_{3} \mathrm{OH}+\mathrm{H}_{2} \mathrm{O} \underset{\mathrm{H}_{2} \mathrm{O}}{\stackrel{\text { cat. }}{\longrightarrow}} 3 \mathrm{H}_{2}+\mathrm{CO}_{2}
$$

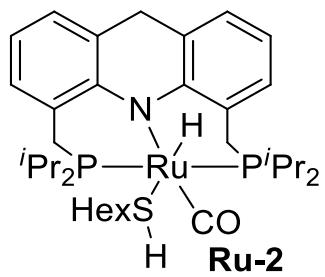

In an $\mathrm{N}_{2}$-filled glovebox, a $90 \mathrm{~mL}$ Fischer-Porter tube was charged with Ru-1 (1.4 mg, $2.5 \mu \mathrm{mol})$, HexSH $(0.35 \mu \mathrm{L}$ in $0.1 \mathrm{~mL}$ methanol, freshly prepared; $2.5 \mu \mathrm{mol})$, methanol $(1.7 \mathrm{~mL})$ and a stirring bar, and the mixture was stirred at room temperature for 1 min to allow for the in situ generation of Ru-2 $(2.5 \mu \mathrm{mol})$ in methanol. ${ }^{11} \mathrm{H}_{2} \mathrm{O}$ $(0.2 \mathrm{~mL})$ was then added and the Fischer-Porter tube was sealed and taken out of the glovebox. The reaction mixture was heated to $150{ }^{\circ} \mathrm{C}$ (oil bath temperature) and stirred at this temperature for $12 \mathrm{~h}$, at which point the pressure gauge of the Fischer-Porter tube reached $\sim 8.5$ bar (under heating). Subsequently, the sealed tube was cooled to room temperature and the observed pressure decreased to $\sim 5$ bar. The Fischer-Porter tube was then connected to an inverted graduated cylinder filled with silicone oil and the tube's valve was slowly opened to allow the gas to flow into the cylinder and displace the oil. After no more gas bubbles were observed, the valve was closed and the collected gas $(470 \mathrm{~mL})$ was analyzed by GC.

\section{Cautions:}

(i) The Fischer-Porter tube should be shielded with an iron net and heated behind a shielder.

(ii) Hydrogen is a flammable gas. Reactions associated with $\mathrm{H}_{2}$ gas should be handled carefully inside proper fume hoods without any flame, spark or static electricity sources nearby.

(iii) Hexanethiol can be smelled when the Fischer-Porter head (adaptor and pressure gauge) is removed, which can also serve as an alarming reagent of leaking. 


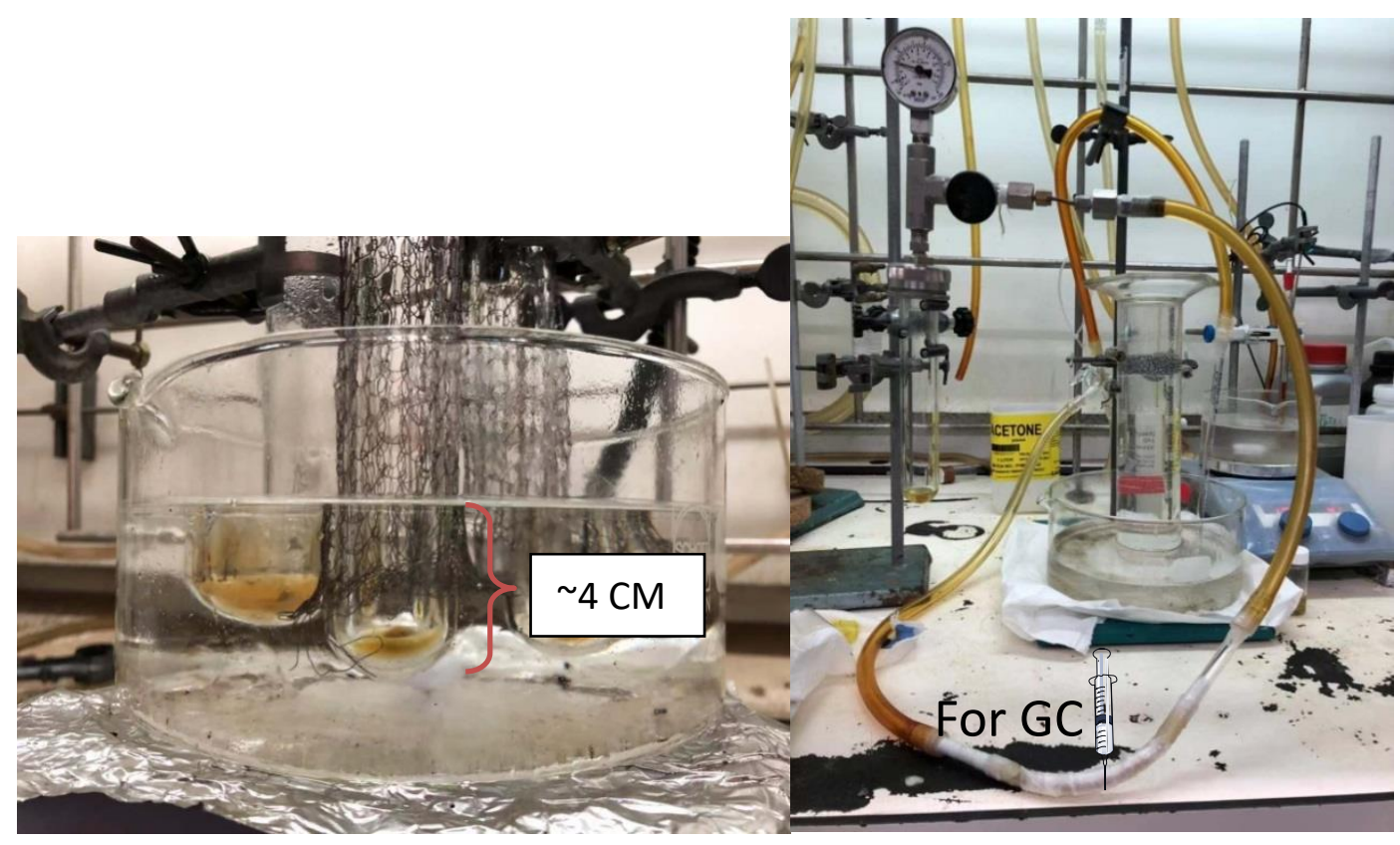

Figure S20. Reaction set-up and gas analysis

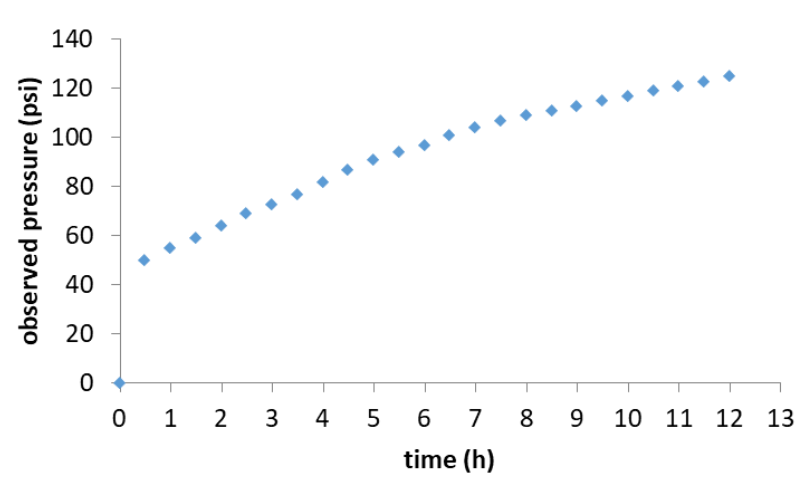

\begin{tabular}{|c|c|c|c|}
\hline time $(\mathrm{h})$ & pressure $(\mathrm{psi})$ & time $(\mathrm{h})$ & pressure $(\mathrm{psi})$ \\
\hline 0 & 0 & 6.5 & 101 \\
\hline 0.5 & 50 & 7 & 104 \\
\hline 1 & 55 & 7.5 & 107 \\
\hline 1.5 & 59 & 8 & 109 \\
\hline 2 & 64 & 8.5 & 111 \\
\hline 2.5 & 69 & 9 & 113 \\
\hline 3 & 73 & 9.5 & 115 \\
\hline 3.5 & 77 & 10 & 117 \\
\hline 4 & 82 & 10.5 & 119 \\
\hline 4.5 & 87 & 11 & 121 \\
\hline 5 & 91 & 11.5 & 123 \\
\hline 5.5 & 94 & 12 & 125 \\
\hline 6 & 97 & & \multicolumn{2}{|l}{} \\
\cline { 2 - 3 }
\end{tabular}

Figure S21. Details on monitored pressure; Due to accuracy limits of the pressure gauge, the values are approximate. 
Long-term reaction with periodic addition of water and methanol: In an $\mathrm{N}_{2}$-filled glovebox, a $90 \mathrm{~mL}$ Fischer-Porter tube was charged with Ru-1 (1.4 mg, $2.5 \mu \mathrm{mol})$, HexSH $(0.35 \mu \mathrm{L}$ in $0.1 \mathrm{~mL}$ methanol, freshly prepared; $2.5 \mu \mathrm{mol})$, methanol (3.5 mL) and a stirring bar, and the mixture was stirred at room temperature for $1 \mathrm{~min}$ to allow for the in situ generation of Ru-2 $(2.5 \mu \mathrm{mol})$ in methanol. ${ }^{11} \mathrm{H}_{2} \mathrm{O}(0.4 \mathrm{~mL})$ was then added and the Fischer-Porter tube was sealed and taken out of the glovebox. The reaction mixture was heated to $150{ }^{\circ} \mathrm{C}$ (oil bath temperature) and stirred at this temperature, usually for $12 \mathrm{~h}$ (1 cycle). After the first heating cycle, the sealed Fischer-Porter tube was cooled to room temperature and connected to an inverted graduated cylinder filled with silicone oil. The tube's valve was carefully opened and the gas was collected in the inverted cylinder. After no more gas bubbles were observed, the valve was closed and the collected gas was analyzed by GC. The Fischer-Porter tube was then reintroduced into the oil bath and the reaction mixture was stirred at $150{ }^{\circ} \mathrm{C}$ for another heating cycle. The above procedure was repeated until $\sim 80 \%$ of the water had been consumed (usually within 3 days). At this point, the Fischer-Porter tube was transferred into the glovebox, and both methanol and water were injected into it to restore the solvent ratio to $3.6 \mathrm{~mL} \mathrm{MeOH} / 0.4 \mathrm{~mL} \mathrm{H}_{2} \mathrm{O}$, based on the amounts of solvent that were consumed, as estimated from the volume of collected gas (trace amounts of $\mathrm{CO}$ were not taken into consideration). The Fischer-Porter tube was then taken out of the glovebox and the above procedure was repeated. Heating of the reaction mixture continued for a total of $592 \mathrm{~h}$, during which $10930 \mathrm{~mL}$ of the $\mathrm{H}_{2} / \mathrm{CO}_{2}$ gas mixture were collected. 


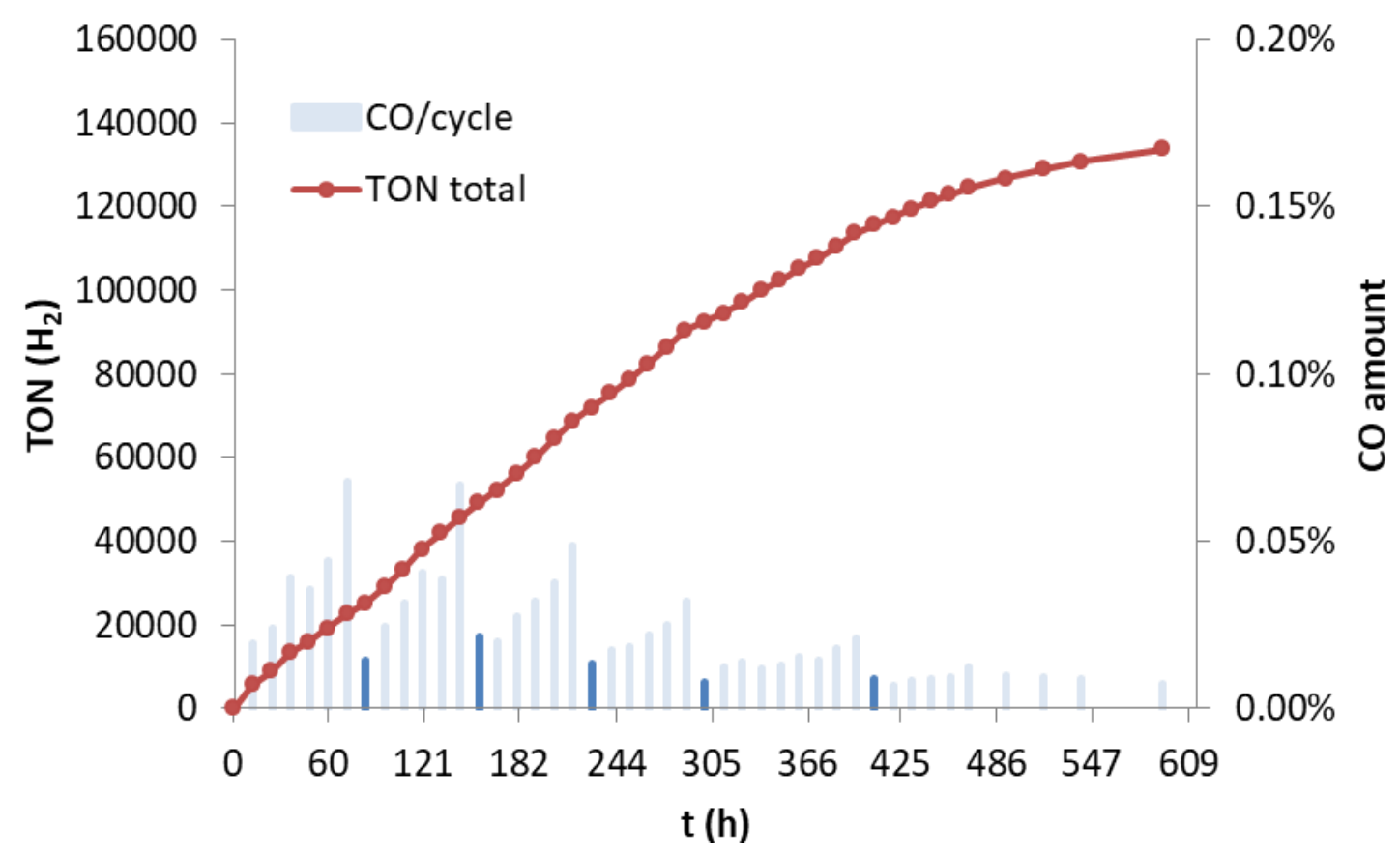

Figure S22. Long term reaction with overall TON $\left(\mathrm{H}_{2}\right)$ of 133837 and trace amount of CO, $592 \mathrm{~h}$ heating in total.

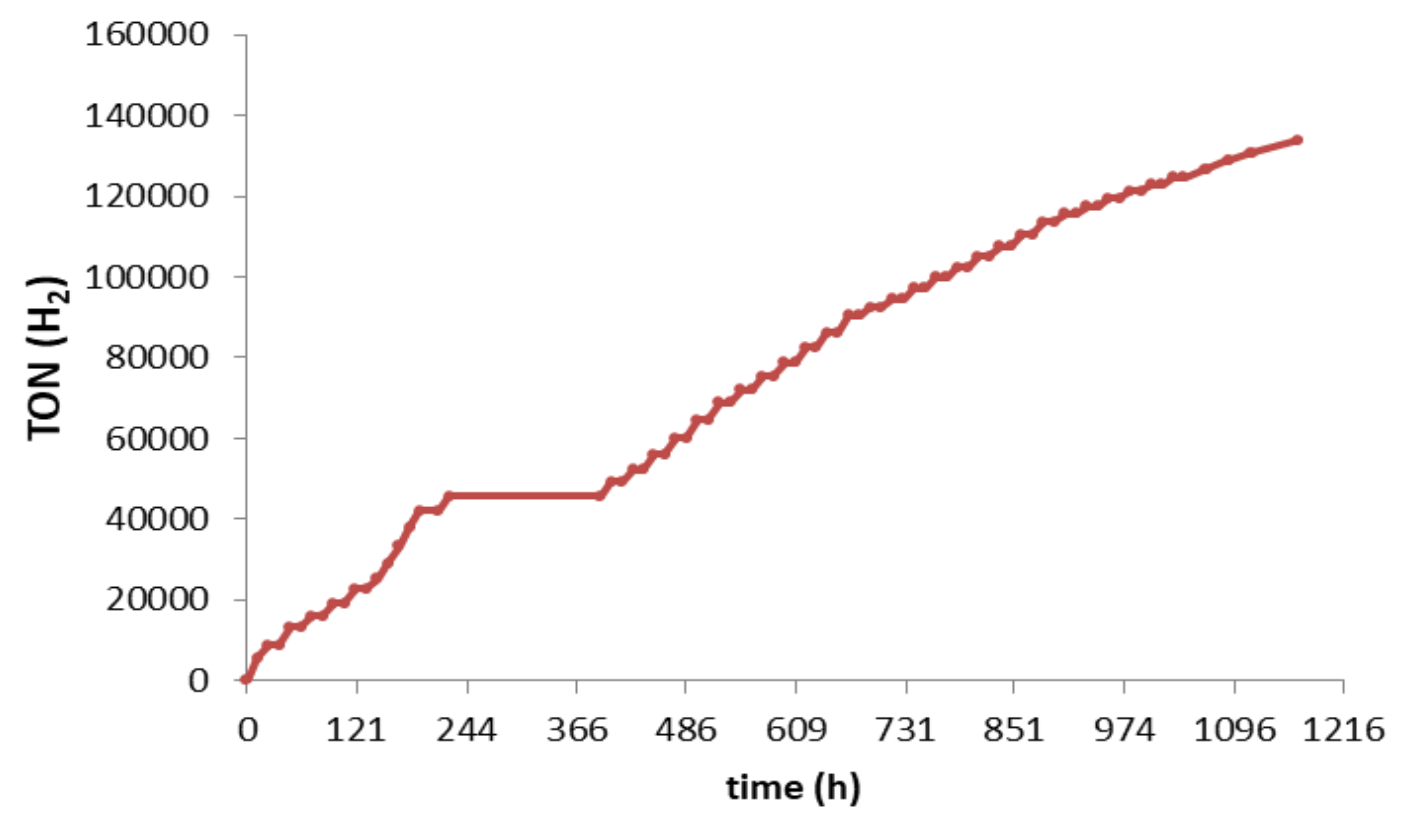

Figure S23. Long term reaction (counting the resting time); Platform means rest at room temperature; $1167 \mathrm{~h}$ in total. 


\begin{tabular}{|c|c|c|c|c|c|c|c|}
\hline reaction time/h & $\mathrm{V}_{\text {gas }} \mathrm{mL} /$ cycle & TON/cycle & TOF/cycle & co/cycle & $\mathrm{V}_{\text {gas }} \mathrm{mL}$ & TON total & TOF average \\
\hline 0 & 0 & 0 & 0 & 0 & 0 & 0 & 0 \\
\hline 12 & 460 & 5633 & 469 & $0.0196 \%$ & 460 & 5633 & 469 \\
\hline 24 & 265 & 3245 & 270 & $0.0239 \%$ & 725 & 8878 & 370 \\
\hline 36 & 360 & 4408 & 367 & $0.0391 \%$ & 1085 & 13286 & 369 \\
\hline 48 & 220 & 2694 & 224 & $0.0357 \%$ & 1305 & 15980 & 333 \\
\hline 60 & 265 & 3245 & 270 & $0.0442 \%$ & 1570 & 19224 & 320 \\
\hline 72 & 280 & 3429 & 286 & $0.0681 \%$ & 1850 & 22653 & 315 \\
\hline 84 & 205 & 2510 & 209 & $0.0147 \%$ & 2055 & 25163 & 300 \\
\hline 96 & 310 & 3796 & 316 & $0.0244 \%$ & 2365 & 28959 & 302 \\
\hline 108 & 355 & 4347 & 362 & $0.0315 \%$ & 2720 & 33306 & 308 \\
\hline 120 & 390 & 4776 & 398 & $0.0408 \%$ & 3110 & 38082 & 317 \\
\hline 132 & 320 & 3918 & 327 & $0.0386 \%$ & 3430 & 42000 & 318 \\
\hline 144 & 290 & 3551 & 296 & $0.0667 \%$ & 3720 & 45551 & 316 \\
\hline 156 & 300 & 3673 & 306 & $0.0214 \%$ & 4020 & 49224 & 316 \\
\hline 168 & 240 & 2939 & 245 & $0.0202 \%$ & 4260 & 52163 & 310 \\
\hline 180 & 320 & 3918 & 327 & $0.0278 \%$ & 4580 & 56082 & 312 \\
\hline 192 & 325 & 3980 & 332 & $0.0323 \%$ & 4905 & 60061 & 313 \\
\hline 204 & 360 & 4408 & 367 & $0.0379 \%$ & 5265 & 64469 & 316 \\
\hline 216 & 350 & 4286 & 357 & $0.0486 \%$ & 5615 & 68755 & 318 \\
\hline 228 & 260 & 3184 & 265 & $0.0135 \%$ & 5875 & 71939 & 316 \\
\hline 240 & 280 & 3429 & 286 & $0.0177 \%$ & 6155 & 75367 & 314 \\
\hline 252 & 270 & 3306 & 276 & $0.0187 \%$ & 6425 & 78673 & 312 \\
\hline 264 & 310 & 3796 & 316 & $0.0219 \%$ & 6735 & 82469 & 312 \\
\hline 276 & 305 & 3735 & 311 & $0.0253 \%$ & 7040 & 86204 & 312 \\
\hline 288 & 340 & 4163 & 347 & $0.0321 \%$ & 7380 & 90367 & 314 \\
\hline 300 & 165 & 2020 & 168 & $0.0080 \%$ & 7545 & 92388 & 308 \\
\hline 312 & 170 & 2082 & 173 & $0.0123 \%$ & 7715 & 94469 & 303 \\
\hline 324 & 215 & 2633 & 219 & $0.0142 \%$ & 7930 & 97102 & 300 \\
\hline 336 & 230 & 2816 & 235 & $0.0118 \%$ & 8160 & 99918 & 297 \\
\hline 348 & 205 & 2510 & 209 & $0.0130 \%$ & 8365 & 102429 & 294 \\
\hline 360 & 220 & 2694 & 224 & $0.0155 \%$ & 8585 & 105122 & 292 \\
\hline 372 & 205 & 2510 & 209 & $0.0143 \%$ & 8790 & 107633 & 289 \\
\hline 384 & 230 & 2816 & 235 & $0.0178 \%$ & 9020 & 110449 & 288 \\
\hline 396 & 265 & 3245 & 270 & $0.0212 \%$ & 9285 & 113694 & 287 \\
\hline 408 & 155 & 1898 & 158 & $0.0090 \%$ & 9440 & 115592 & 283 \\
\hline 420 & 150 & 1837 & 153 & $0.0070 \%$ & 9590 & 117429 & 280 \\
\hline 432 & 160 & 1959 & 163 & $0.0086 \%$ & 9750 & 119388 & 276 \\
\hline 444 & 150 & 1837 & 153 & $0.0091 \%$ & 9900 & 121224 & 273 \\
\hline 456 & 140 & 1714 & 143 & $0.0095 \%$ & 10040 & 122939 & 270 \\
\hline 468 & 130 & 1592 & 133 & $0.0126 \%$ & 10170 & 124531 & 266 \\
\hline 492 & 180 & 2204 & 92 & $0.0101 \%$ & 10350 & 126735 & 258 \\
\hline 516 & 175 & 2143 & 89 & $0.0097 \%$ & 10525 & 128878 & 250 \\
\hline 540 & 155 & 1898 & 79 & $0.0091 \%$ & 10680 & 130776 & 242 \\
\hline 592 & 250 & 3061 & 59 & $0.0075 \%$ & 10930 & 133837 & 226 \\
\hline
\end{tabular}

Figure S24. Details of the long-term reaction; $\mathrm{MeOH}$ and $\mathrm{H}_{2} \mathrm{O}$ was injected before the reaction cycle colored in light purple. In order, the following quantities are added: 1. $0.34 \mathrm{~mL} \mathrm{H} \mathrm{H}_{2} \mathrm{O}+0.77 \mathrm{~mL} \mathrm{MeOH}$; 2. $0.34 \mathrm{~mL} \mathrm{H}_{2} \mathrm{O}+0.77 \mathrm{~mL} \mathrm{MeOH} ; 3.0 .35 \mathrm{~mL}$ $\mathrm{H}_{2} \mathrm{O}+0.78 \mathrm{~mL} \mathrm{MeOH}$; 4. $0.32 \mathrm{~mL} \mathrm{H} \mathrm{H}_{2} \mathrm{O}+0.73 \mathrm{~mL} \mathrm{MeOH} ; 5.0 .35 \mathrm{~mL} \mathrm{H} \mathrm{H}_{2} \mathrm{O}+0.79$ $\mathrm{mL} \mathrm{MeOH}$. 


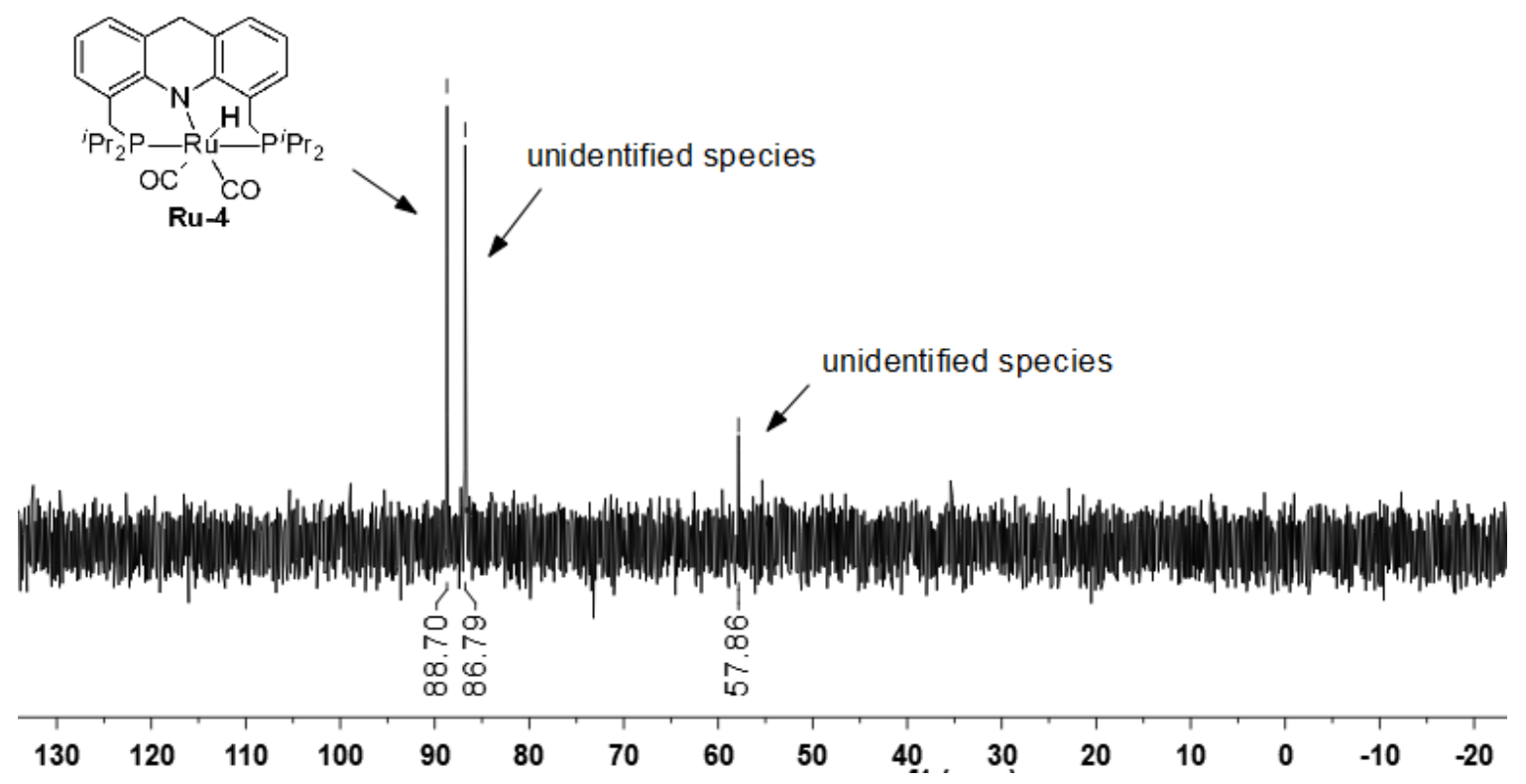

Figure S25. ${ }^{31} \mathrm{P}$ NMR of the resulting species in benzene- $d 6$ after $592 \mathrm{~h}$ heating (from the 9:1 system); Considerable amount of $\mathbf{R u - 4}$, the resting state of the catalyst, was detected without the observation of free ligand. 

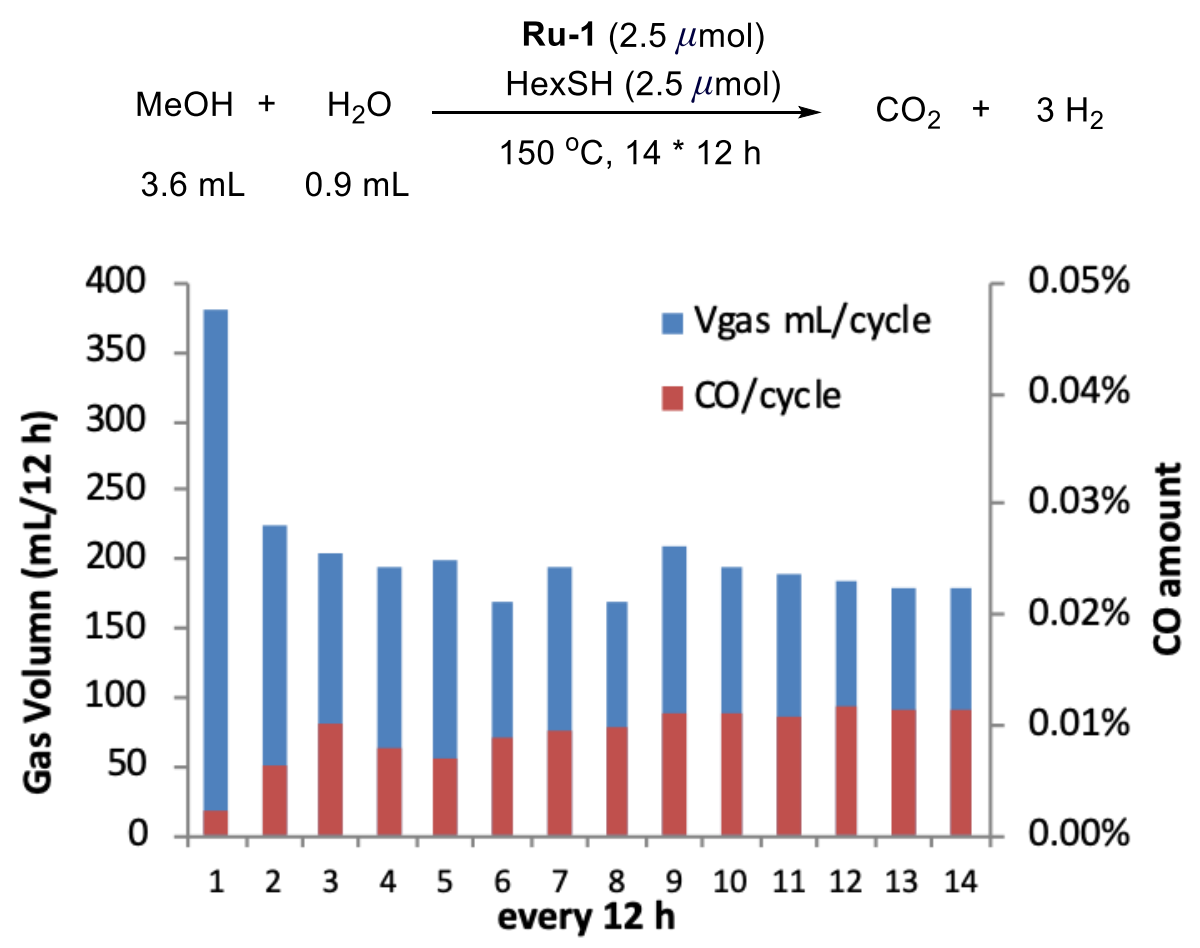

\begin{tabular}{|c|c|c|}
\hline Cycle/12 $\mathrm{h}$ & $\mathrm{V}_{\text {gas }} \mathrm{mL} /$ cycle & CO/cycle \\
\hline 1 & 380 & $0.0022 \%$ \\
\hline 2 & 225 & $0.0064 \%$ \\
\hline 3 & 205 & $0.0100 \%$ \\
\hline 4 & 195 & $0.0080 \%$ \\
\hline 5 & 200 & $0.0068 \%$ \\
\hline 6 & 170 & $0.0087 \%$ \\
\hline 7 & 195 & $0.0096 \%$ \\
\hline 8 & 170 & $0.0099 \%$ \\
\hline 9 & 210 & $0.0110 \%$ \\
\hline 10 & 195 & $0.0111 \%$ \\
\hline 11 & 190 & $0.0106 \%$ \\
\hline 12 & 185 & $0.0118 \%$ \\
\hline 13 & 180 & $0.0113 \%$ \\
\hline 14 & 180 & $0.0112 \%$ \\
\hline
\end{tabular}

Figure S26. Details of the short continuous reaction in $3.6 \mathrm{~mL} \mathrm{MeOH}$ and $0.9 \mathrm{~mL}$ $\mathrm{H}_{2} \mathrm{O}$. 


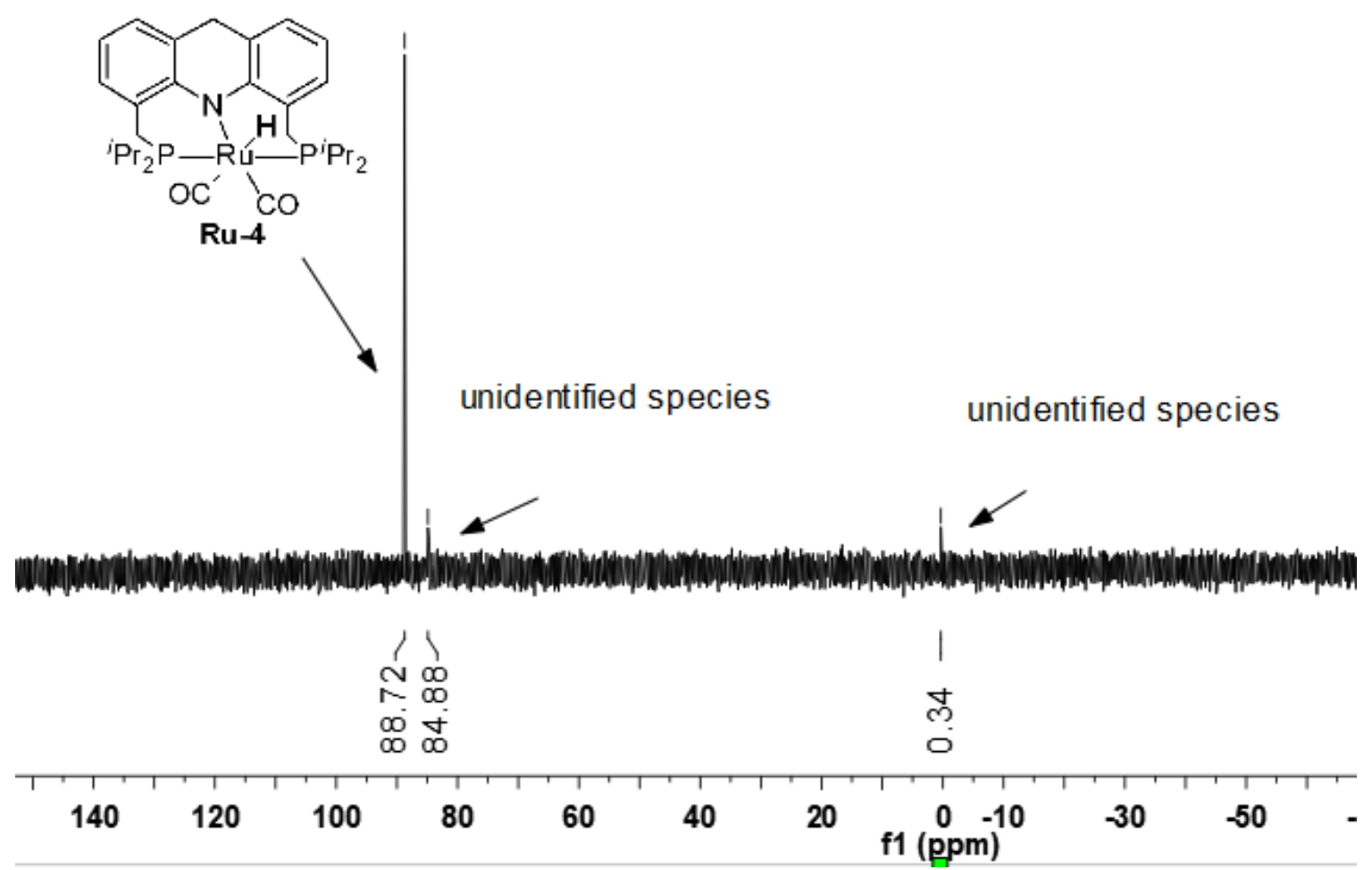

Figure S27. ${ }^{31} \mathrm{P}$ NMR of the resulting species in benzene- $d 6$ after $302 \mathrm{~h}$ heating (from 6:1 system), indicating the major species is still $\mathbf{R u}-\mathbf{4}$, the resting state of the catalyst. 
Ru-1 $(25 \mu \mathrm{mol})$

$\mathrm{MeOH}+\mathrm{H}_{2} \mathrm{O} \underset{150^{\circ} \mathrm{C}}{\stackrel{\mathrm{HexSH}(25 \mu \mathrm{mol})}{\longrightarrow}} \mathrm{CO}_{2}+3 \mathrm{H}_{2}$

$7.2 \mathrm{~mL} \quad 1.8 \mathrm{~mL}$

- Vgas $\mathrm{mL} /$ cycle $\quad \mathrm{CO} /$ cycle

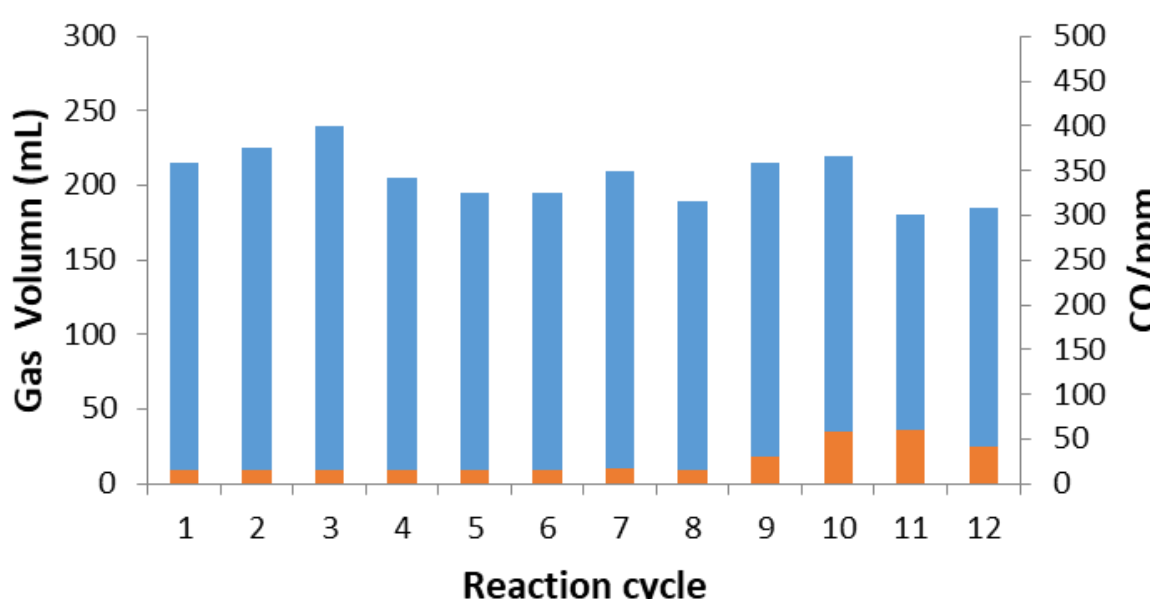

\begin{tabular}{|c|c|c|c|c|}
\hline Cycle & $\begin{array}{c}\text { reaction period } \\
\min \end{array}$ & $\mathrm{t} / \mathrm{min}$ & $\mathrm{V}_{\text {gas }} \mathrm{mL} /$ cycle & CO/cycle \\
\hline 1 & 60 & 60 & 215 & $<15$ p.p.m. \\
\hline 2 & 60 & 120 & 225 & $<15$ p.p.m. \\
\hline 3 & 70 & 190 & 240 & $<15$ p.p.m. \\
\hline 4 & 60 & 250 & 205 & $<15$ p.p.m. \\
\hline 5 & 70 & 320 & 195 & $<15$ p.p.m. \\
\hline 6 & 70 & 390 & 195 & $<15$ p.p.m. \\
\hline 7 & 90 & 480 & 210 & $<20$ p.p.m. \\
\hline 8 & 90 & 570 & 190 & $<15$ p.p.m. \\
\hline 9 & 120 & 690 & 215 & 30 p.p.m. \\
\hline 10 & 120 & 810 & 220 & 58 p.p.m. \\
\hline 11 & 120 & 930 & 180 & 61 p.p.m. \\
\hline 12 & 120 & 1050 & 185 & 41 p.p.m. \\
\hline
\end{tabular}

Figure S28. Details of the short continuous reaction in $7.2 \mathrm{~mL} \mathrm{MeOH}$ and $1.8 \mathrm{~mL}$ $\mathrm{H}_{2} \mathrm{O}$ with 0.014 mol\% Ru-1. 


\section{Representative GC spectra}

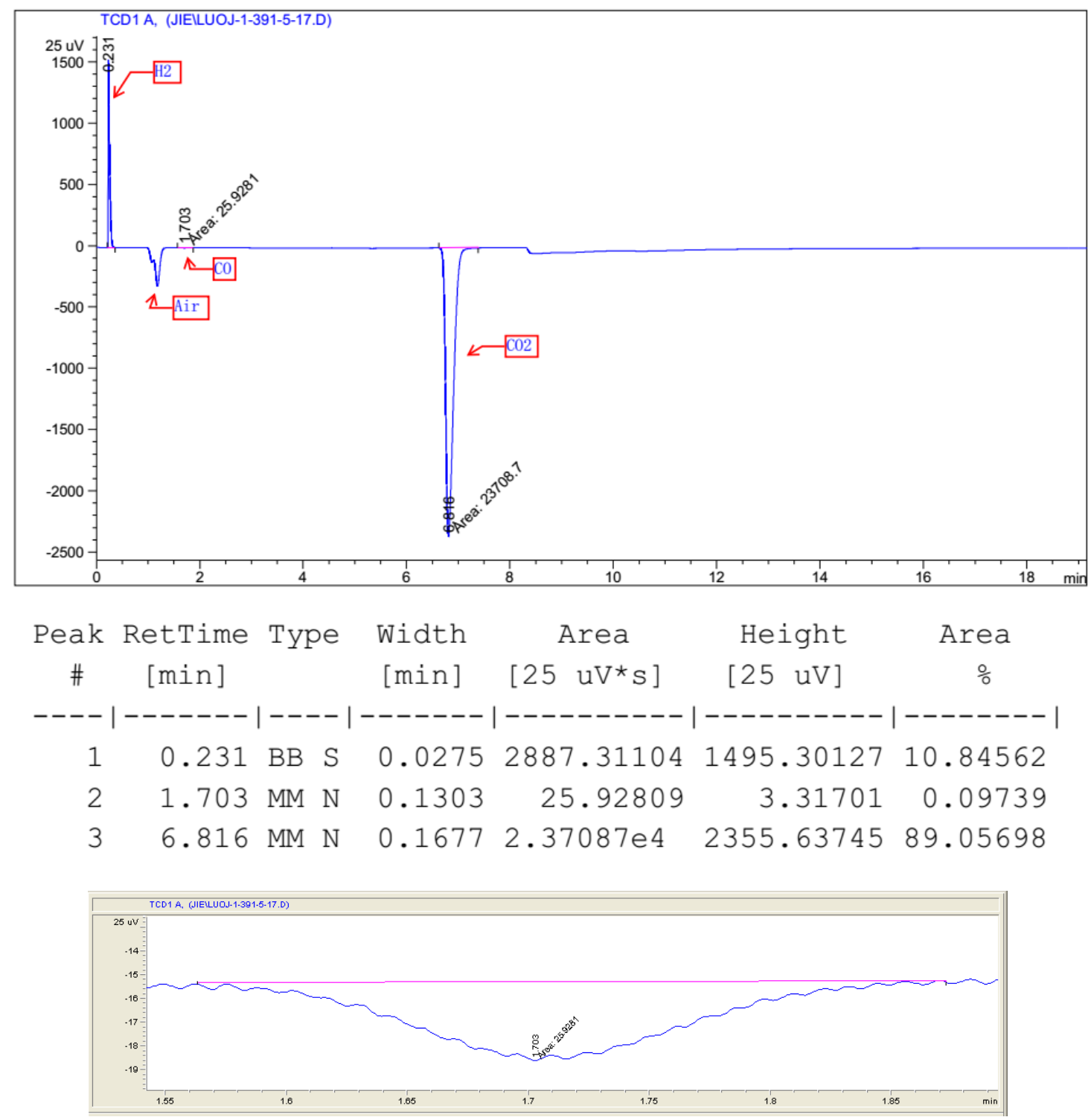

Figure S29. GC-gas trace of collected gas $\mathrm{H}_{2} / \mathrm{CO}_{2}=3$ (3.08): 1 with detectable $\mathrm{CO}$ $(0.0365 \%)$. 


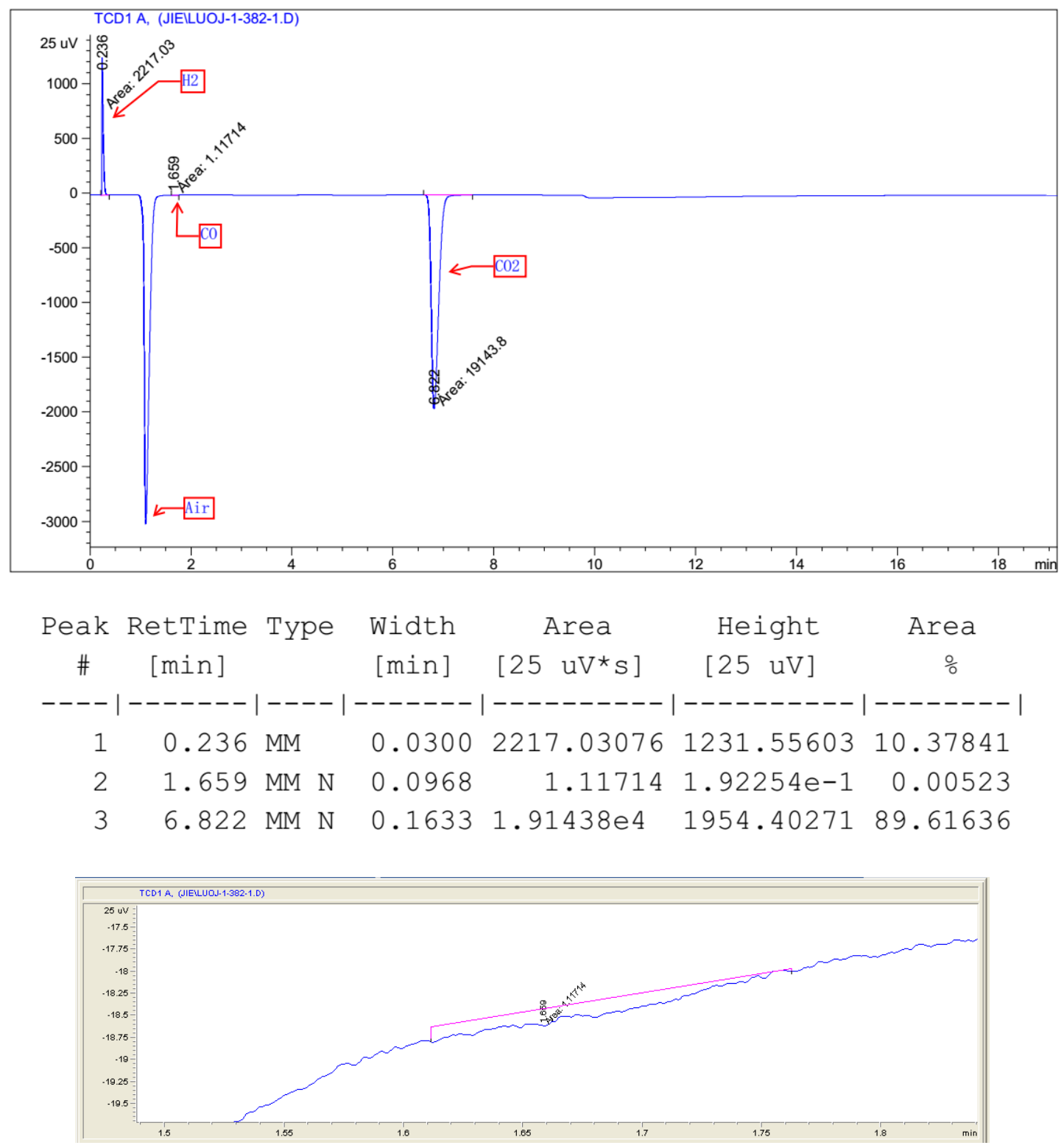

Figure S30. GC trace of collected $\mathrm{H}_{2} / \mathrm{CO}_{2}=3$ (2.94): 1 with $\mathrm{CO}(20 \mathrm{ppm})$ around detection limit. 


\section{Condition screening}

$\mathbf{R u}(2.5 \mu \mathrm{mol})$ $\mathrm{MeOH}+\mathrm{H}_{2} \mathrm{O} \underset{150{ }^{\circ} \mathrm{C}, 12 \mathrm{~h}}{\stackrel{\mathrm{HexSH}(2.5 \mu \mathrm{mol})}{\longrightarrow}} \mathrm{CO}_{2}+3 \mathrm{H}_{2}$

$1.8 \mathrm{~mL} \quad 0.2 \mathrm{~mL}$

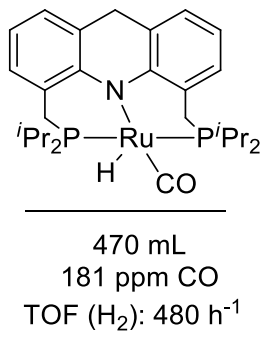

$$
\begin{aligned}
& 240 \mathrm{~mL} \\
& 105 \mathrm{ppm} \mathrm{CO} \\
& \text { rof }\left(\mathrm{H}_{2}\right): 245 \mathrm{~h}^{-1} \\
& \text { beplace } \mathrm{N}_{2} \text { with } \\
& \text { before the reaction }
\end{aligned}
$$

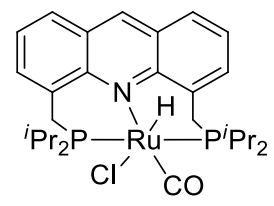

$120 \mathrm{~mL}$

113 ppm CO

TOF $\left(\mathrm{H}_{2}\right): 122 \mathrm{~h}^{-1}$

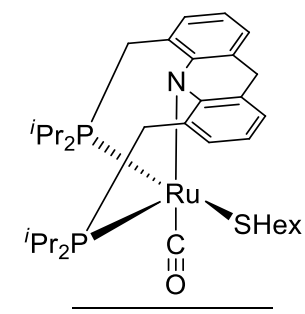

$455 \mathrm{~mL}$

110 ppm CO

TOF $\left(\mathrm{H}_{2}\right): 464 \mathrm{~h}^{-1}$

without thiol

Figure S31. Catalyst screening.
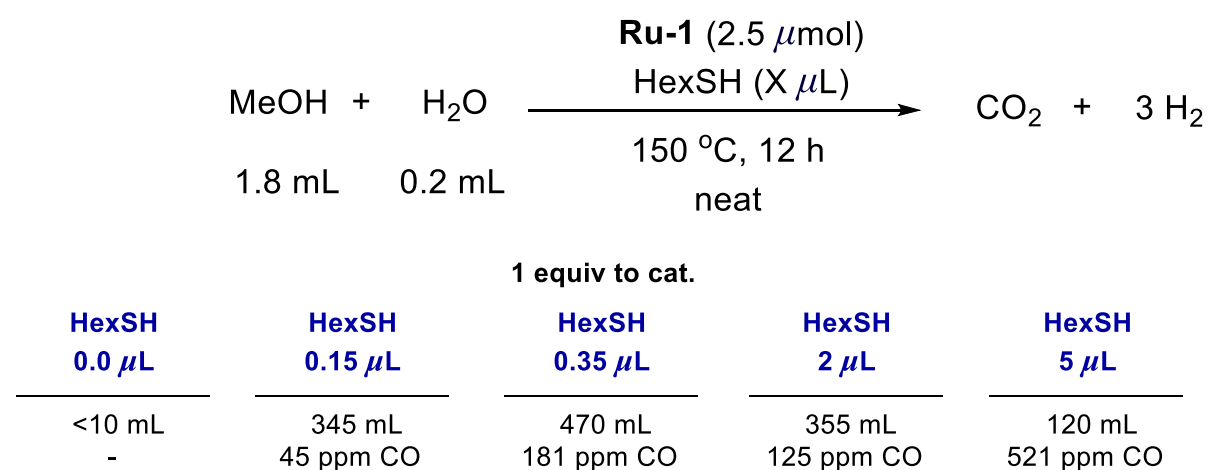

1 equiv to cat.

Figure S32. Effect of amounts of thiol on the reaction. 


$$
\mathrm{MeOH}+\mathrm{H}_{2} \mathrm{O} \underset{150^{\circ} \mathrm{C}, 12 \mathrm{~h}}{\stackrel{\text { Ru-1 }(2.5 \mu \mathrm{mol})}{\text { additive }(2.5 \mu \mathrm{mol})} \rightarrow \mathrm{CO}_{2}+3 \mathrm{H}_{2}}
$$

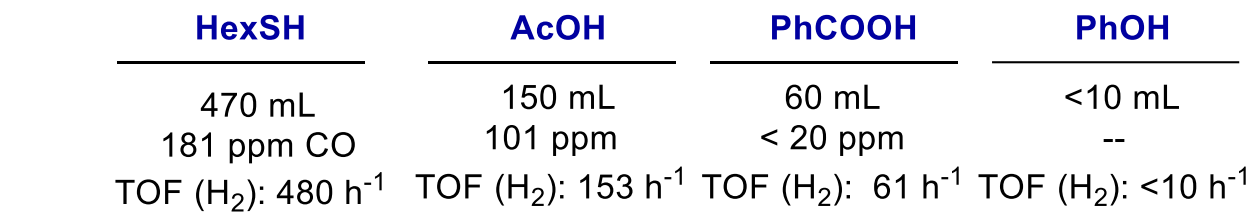

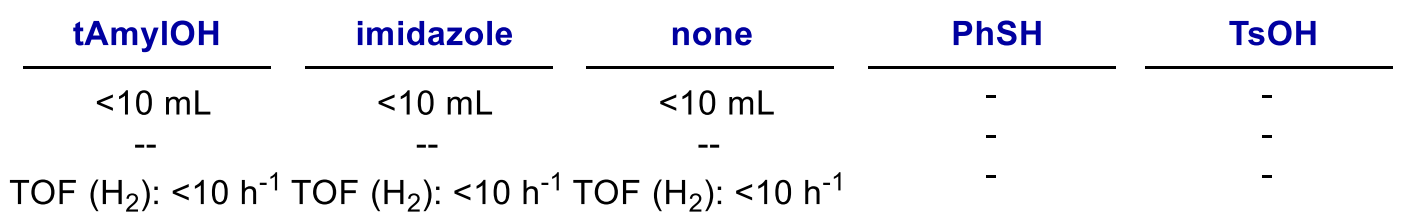

Figure S33. Screening of additives.

\section{Control experiments}

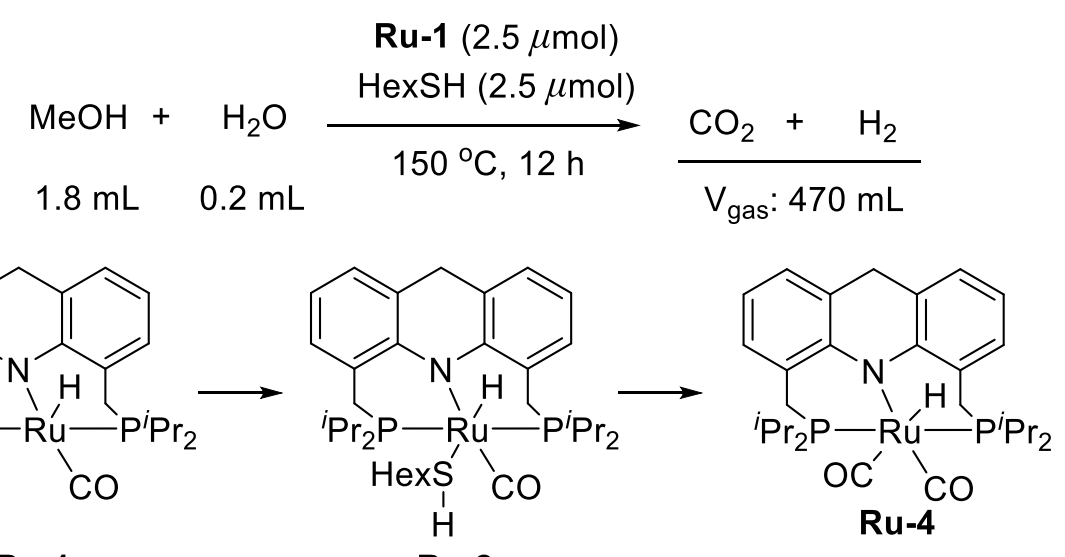

Ru-1

Ru-2

The Fischer-Porter tube was sealed and transfered back to the glove box after the reaction (following the general procedure). The resulting solution was directly measured by ${ }^{31} \mathrm{P}$ NMR, indicating that a new species was formed. Then the solvent (methanol and water) was directly removed under vacuum and the resulting solid was dissolved in THF for NMR analysis. Both the signals of ${ }^{31} \mathrm{P}$ NMR and ${ }^{1} \mathrm{H}$ NMR (hydride peak) match a biscarbonyl ruthenium acridine complex Ru-4. Note the characterization of Ru-2 was done in $\mathrm{MeOH}-d 4$ directly mixing Ru-1 and 1 equiv hexanethiol. ${ }^{11}$ 


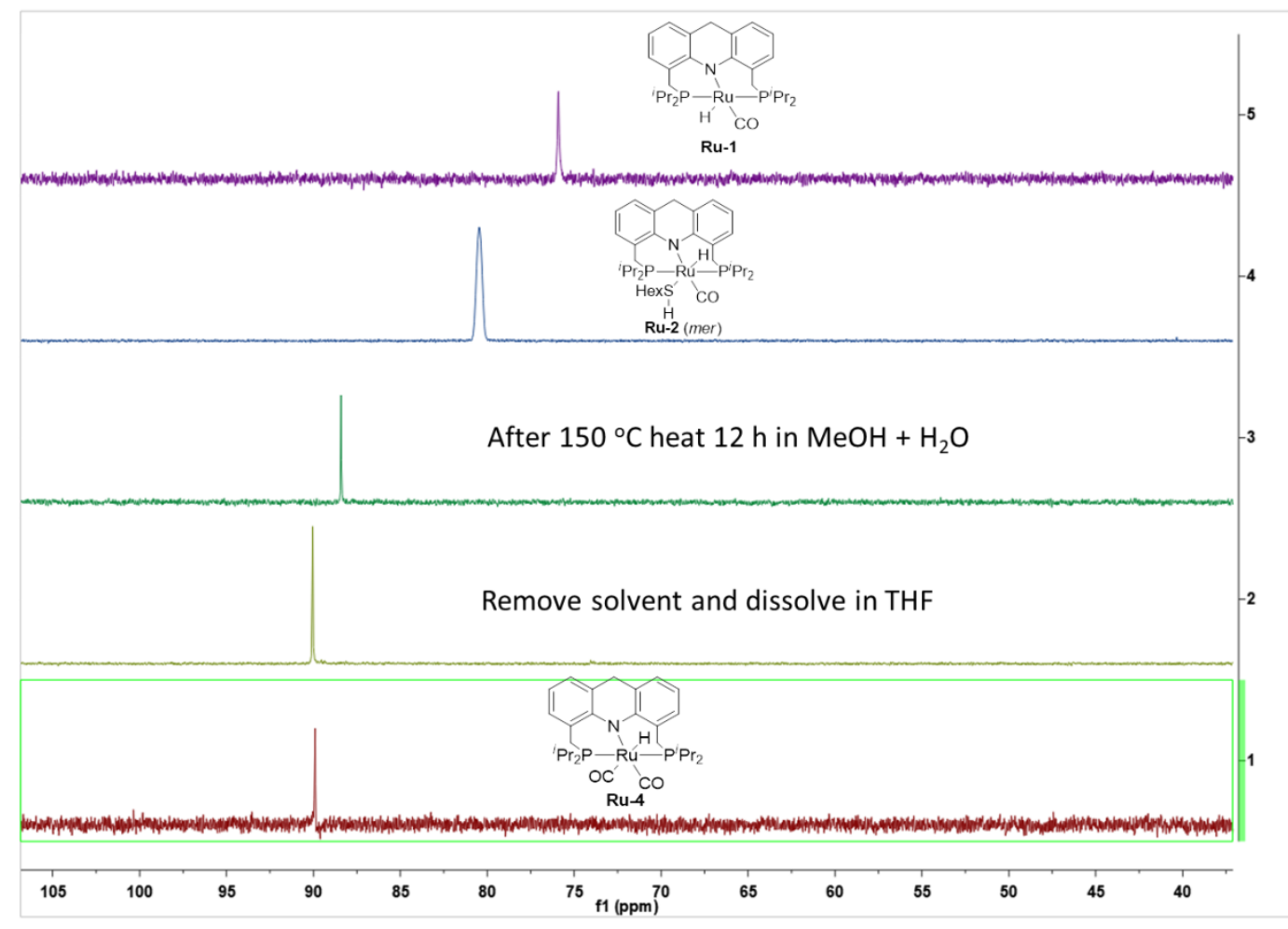

Figure S34. ${ }^{31} \mathrm{P}$ NMR of resulting species after reaction.

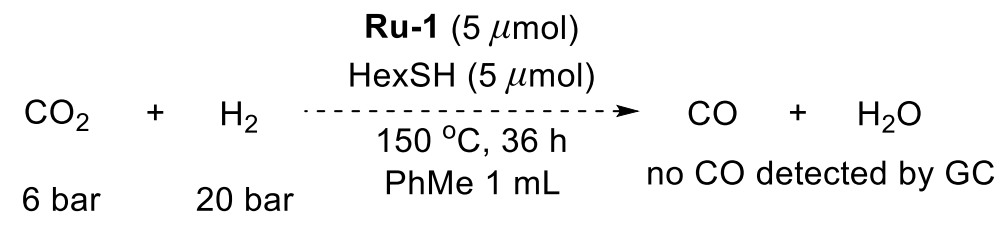

In a $\mathrm{N}_{2}$ glove box, Ru-1 $(2.8 \mathrm{mg}, 5 \mu \mathrm{mol}), \mathrm{HexSH}(0.7 \mu \mathrm{L}, 5 \mu \mathrm{mol}$, by micro-syringe) and toluene- $d_{8}(0.6 \mathrm{~mL})$ were added to a $30 \mathrm{~mL}$ steel autoclave fitted with a Teflon sleeve. The autoclave was taken out of the glove box and pressurized with 6 bar $\mathrm{CO}_{2}$ and 20 bar $\mathrm{H}_{2}$ sequentially. After heating at $150{ }^{\circ} \mathrm{C}$ for $36 \mathrm{~h}$ with stirring, the steel autoclave was cooled in a cold water bath for $30 \mathrm{~min}$. Then the gas was vented off carefully and analyzed by GC. The resulting solution was analyzed by GCMS and NMR. However, $\mathrm{CO}$ and $\mathrm{MeOH}$ were not detected.
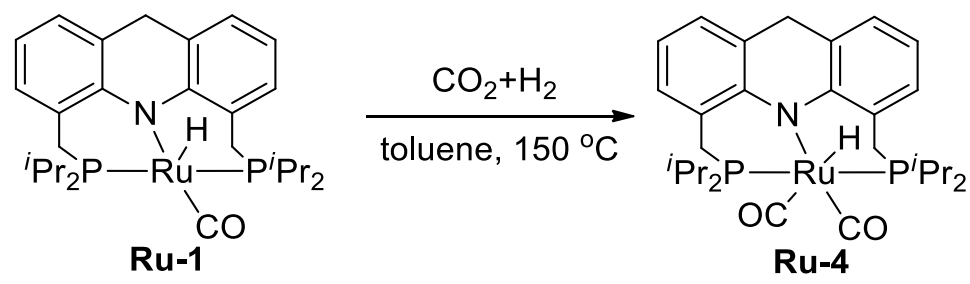
In a $\mathrm{N}_{2}$ glove box, Ru-1 (0.012 g, $\left.0.02 \mathrm{mmol}\right)$ and toluene- $d_{8}(0.6 \mathrm{~mL})$ were added to a $30 \mathrm{~mL}$ steel autoclave fitted with a Teflon sleeve. The autoclave was taken out of the glove box and pressurized with 6 bar $\mathrm{CO}_{2}$ and 20 bar $\mathrm{H}_{2}$ sequentially. After heating at $150{ }^{\circ} \mathrm{C}$ for $72 \mathrm{~h}$ with stirring, the steel autoclave was cooled in a cold water bath for $30 \mathrm{~min}$. Then the gas was vented off carefully and analyzed by GC. No CO was detected. The steel autoclave was transferred back into the $\mathrm{N}_{2}$ glove box and the resulting solution was directly transferred to a J. Young NMR tube for NMR analysis. Both ${ }^{31} \mathrm{P}$ NMR and ${ }^{1} \mathrm{H}$ NMR indicate two major new species generated, one of which is Ru-4 as signed below. However, after further heating the J. Young NMR tube at $150{ }^{\circ} \mathrm{C}$ for $2 \mathrm{~h}$, the other ruthenium species also converted into $\mathbf{R u}-\mathbf{4}$ as indicated by ${ }^{31} \mathrm{P}$ NMR. These results indicate $\mathbf{R u}-\mathbf{1}$ can react with $\mathrm{CO}_{2}$ and $\mathrm{H}_{2}$ to generate $\mathbf{R u}-\mathbf{4}$. However, Ru-4 is not active enough to conduct further catalysis with $\mathrm{CO}_{2}$ and $\mathrm{H}_{2}$.

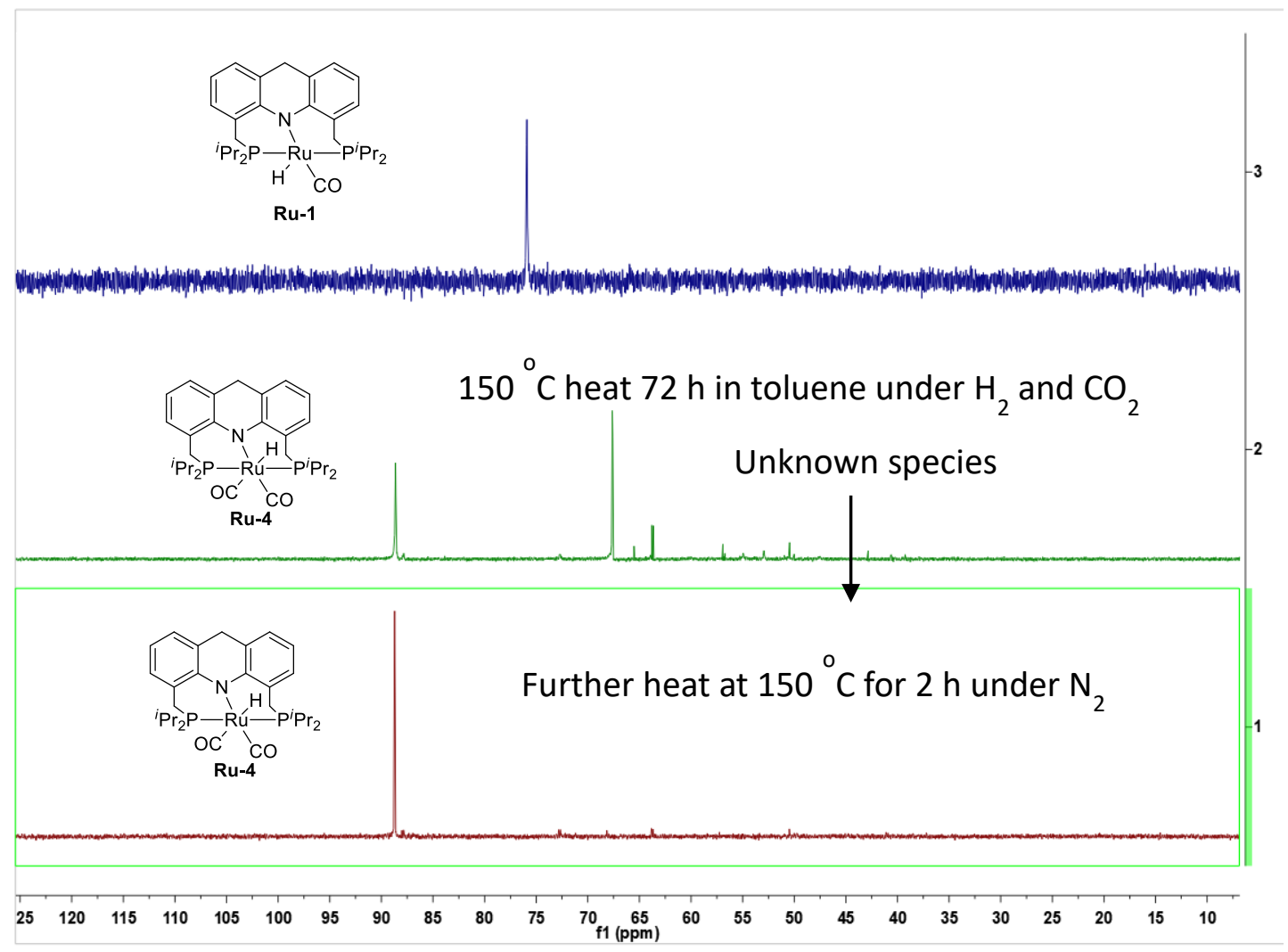

Figure S35. ${ }^{31} \mathrm{P}$ NMR spectra of the resulting species after heating under $\mathrm{CO}_{2}$ and $\mathrm{H}_{2}$. 


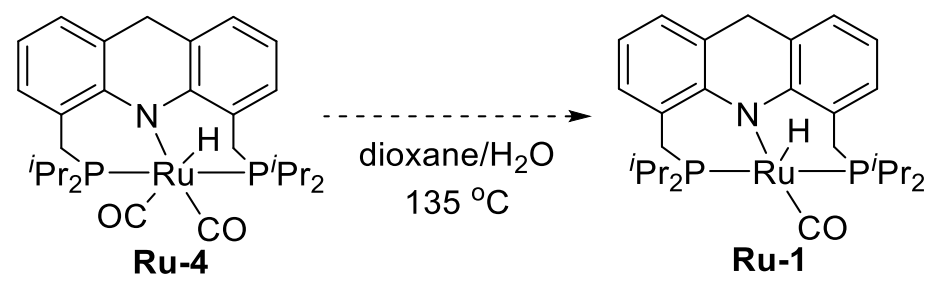

In a $\mathrm{N}_{2}$ glove box, Ru-4 $(3.0 \mathrm{mg}, 5 \mu \mathrm{mol})$, dioxane $(0.5 \mathrm{~mL})$ and water $(0.1 \mathrm{~mL})$ were added to a J. Young NMR tube. The NMR tube was taken out of the box and heated at $135{ }^{\circ} \mathrm{C}$ for $24 \mathrm{~h}$, after which it was cooled to room temperature and measured by ${ }^{31} \mathrm{P}$ NMR and ${ }^{1} \mathrm{H}$ NMR. Two species were detected by NMR, one of which was Ru-4 as signed below. The NMR tube was transferred back into the box and dioxane and water was removed under vacuum. Then toluene $(0.5 \mathrm{~mL})$ was added and the NMR tube was taken out of the box and measured again. Both ${ }^{31} \mathrm{P}$ NMR and ${ }^{1} \mathrm{H}$ NMR indicated that no Ru-1 was regenerated, eliminating the possibility of water-gas-shift reaction in the system.

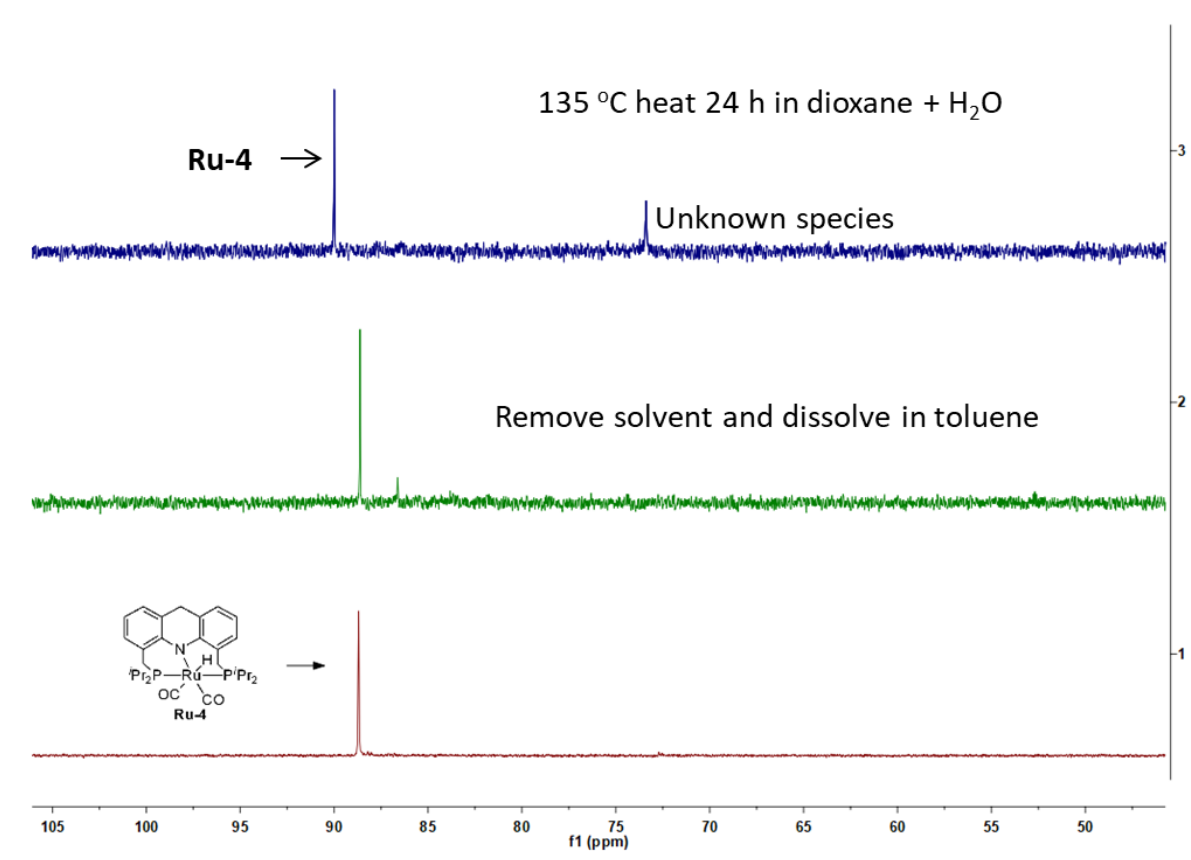

Figure S36. ${ }^{31} \mathrm{P}$ NMR spectra of the resulting species after heating with water. 


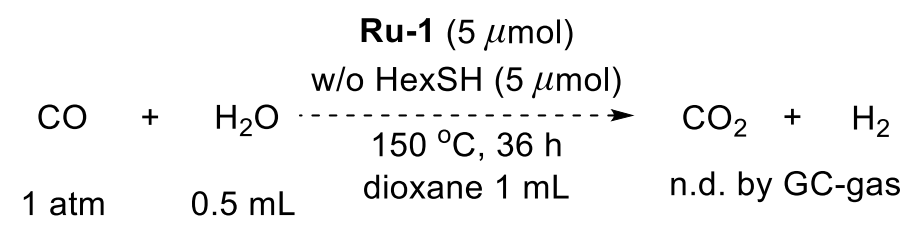

In a $\mathrm{N}_{2}$ glove box, Ru-1 (2.8 mg, $\left.5 \mu \mathrm{mol}\right)$, HexSH $(0$ or $0.7 \mu \mathrm{L}, 0$ or $5 \mu \mathrm{mol}$, by micro-syringe), water $(0.5 \mathrm{~mL})$ and dioxane $(1 \mathrm{~mL})$ were added to a $90 \mathrm{~mL}$ Fischer-Porter tube. The tube was taken out of the glove box and evacuated until some bubbling of the solvent was observed. Then it was refilled with CO. After heating at $150{ }^{\circ} \mathrm{C}$ for $36 \mathrm{~h}$ with stirring, the Fischer-Porter tube was cooled to room temperature. The gauge of Fischer-Porter tube indicated no pressure was generated after heating. The gas inside the tube was analyzed by GC. However, $\mathrm{CO}_{2}$ and $\mathrm{H}_{2}$ were not detected.

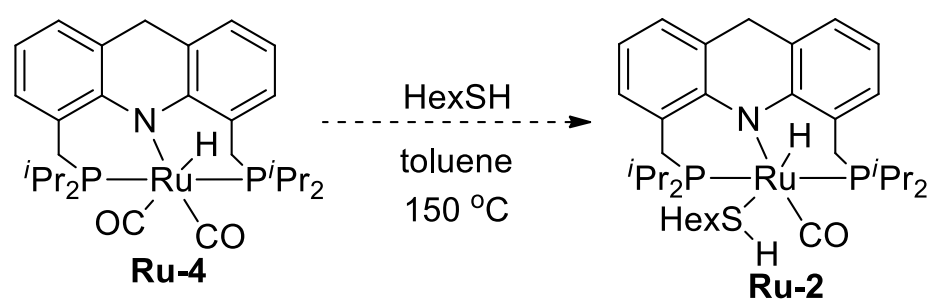

In a $\mathrm{N}_{2}$ glove box, Ru-4 (3.0 mg, $\left.5 \mu \mathrm{mol}\right), \operatorname{HexSH}(0.7 \mu \mathrm{L}, 5.0 \mu \mathrm{mol}$, by microsyringe), toluene $(0.6 \mathrm{~mL})$ were added to a J. Young NMR tube. The NMR tube was taken out of the box and heated at $150{ }^{\circ} \mathrm{C}$ under Ar flow for $18 \mathrm{~h}$, after which it was cooled to room temperature and measured by ${ }^{31} \mathrm{P}$ NMR and ${ }^{1} \mathrm{H} \mathrm{NMR}$, indicating no change of Ru-4. 


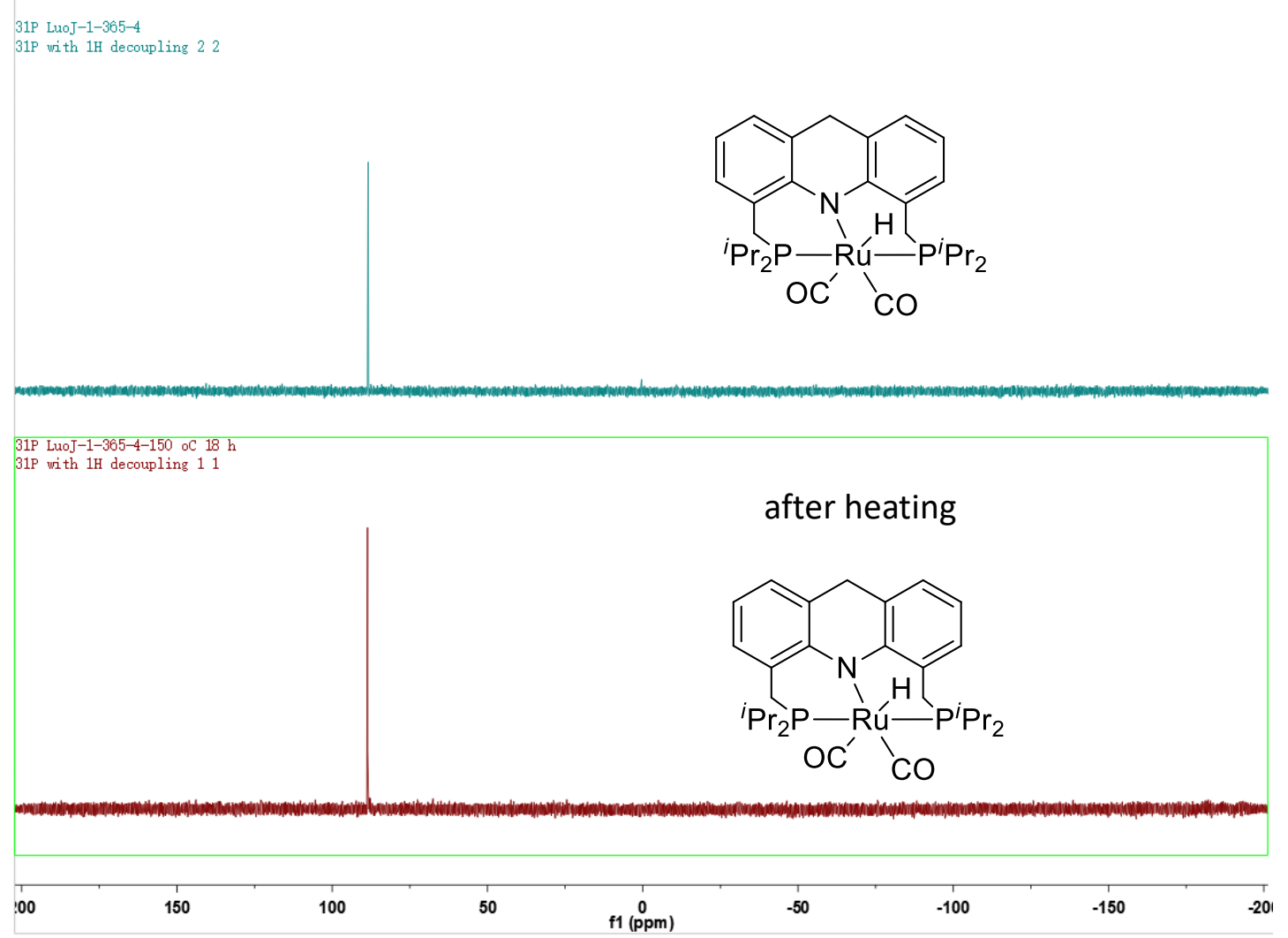

Figure S37. ${ }^{31} \mathrm{P}$ NMR spectra of the resulting species after heating with thiol.
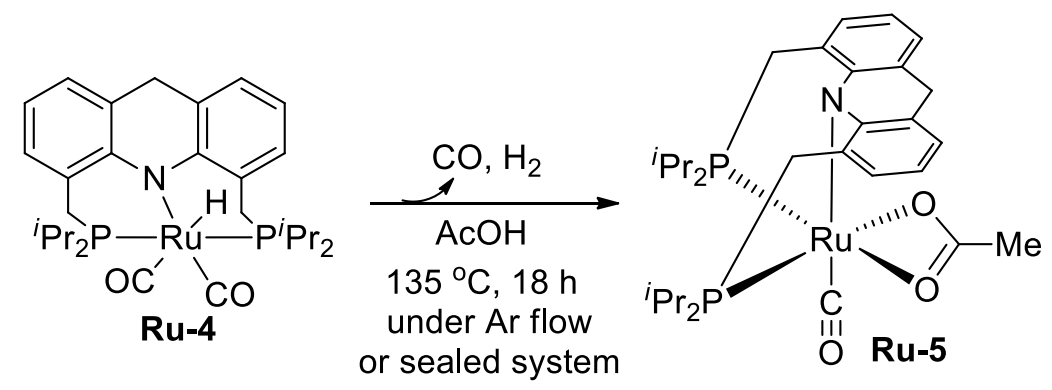

In a $\mathrm{N}_{2}$ glove box, Ru-4 (0.012 g, $\left.0.02 \mathrm{mmol}\right)$, acetic acid $(0.5 \mathrm{~mL})$ were added to a J. Young NMR tube. The NMR tube was taken out of the box and heated at $135{ }^{\circ} \mathrm{C}$ for 10 min. After cooling down to room temperature, the NMR tube was transferred back to the glove box and the cap of NMR tube was sealed with film. Then the cap was carefully opened and the released gas was directly collected by a micro-syringe for GC analysis. Both $\mathrm{CO}$ and $\mathrm{H}_{2}$ were detected (Figure S38). The NMR tube was taken out of the box and further heated under Ar flow for $18 \mathrm{~h}$, after which it was cooled to room temperature and measured by ${ }^{31} \mathrm{P}$ NMR and ${ }^{1} \mathrm{H}$ NMR, indicating a major new species generated as shown below (incomplete conversion was observed in a parallel 
experiment in closed system). The NMR tube was transferred back into the box and acetic acid was thoroughly removed under vacuum. Then benzene- $d_{6}(0.5 \mathrm{~mL})$ was added and the NMR tube was measured again, indicating the generation of a ruthenium acetate complex. The result proves the lability of the second $\mathrm{CO}$ on the ruthenium center. The characteristic data of $\mathbf{R u - 1 6}$ is in accordance with the reported one. ${ }^{1}$

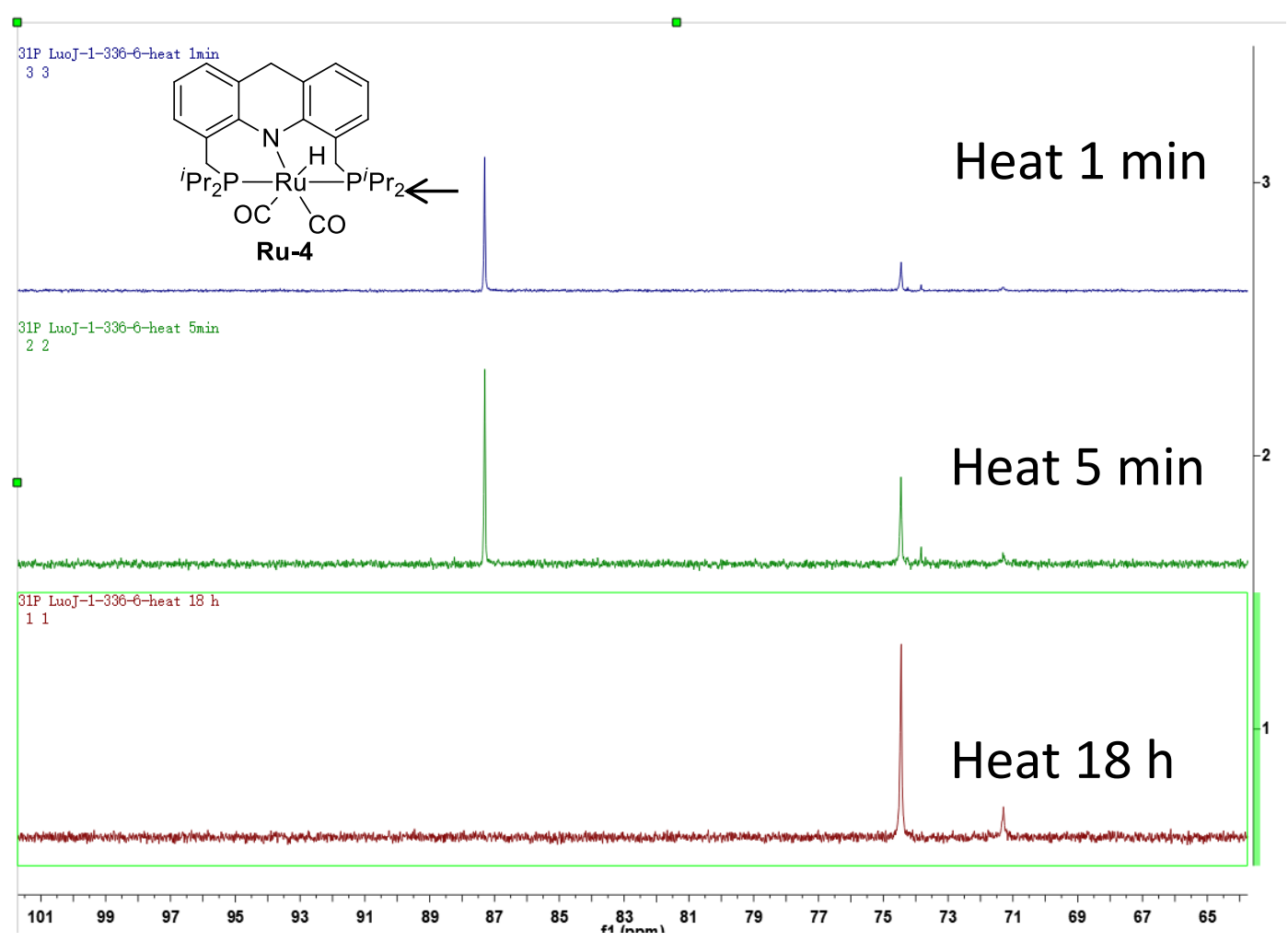

Figure S38. ${ }^{31} \mathrm{P}$ NMR spectra of the resulting species after heating in acetic acid. 


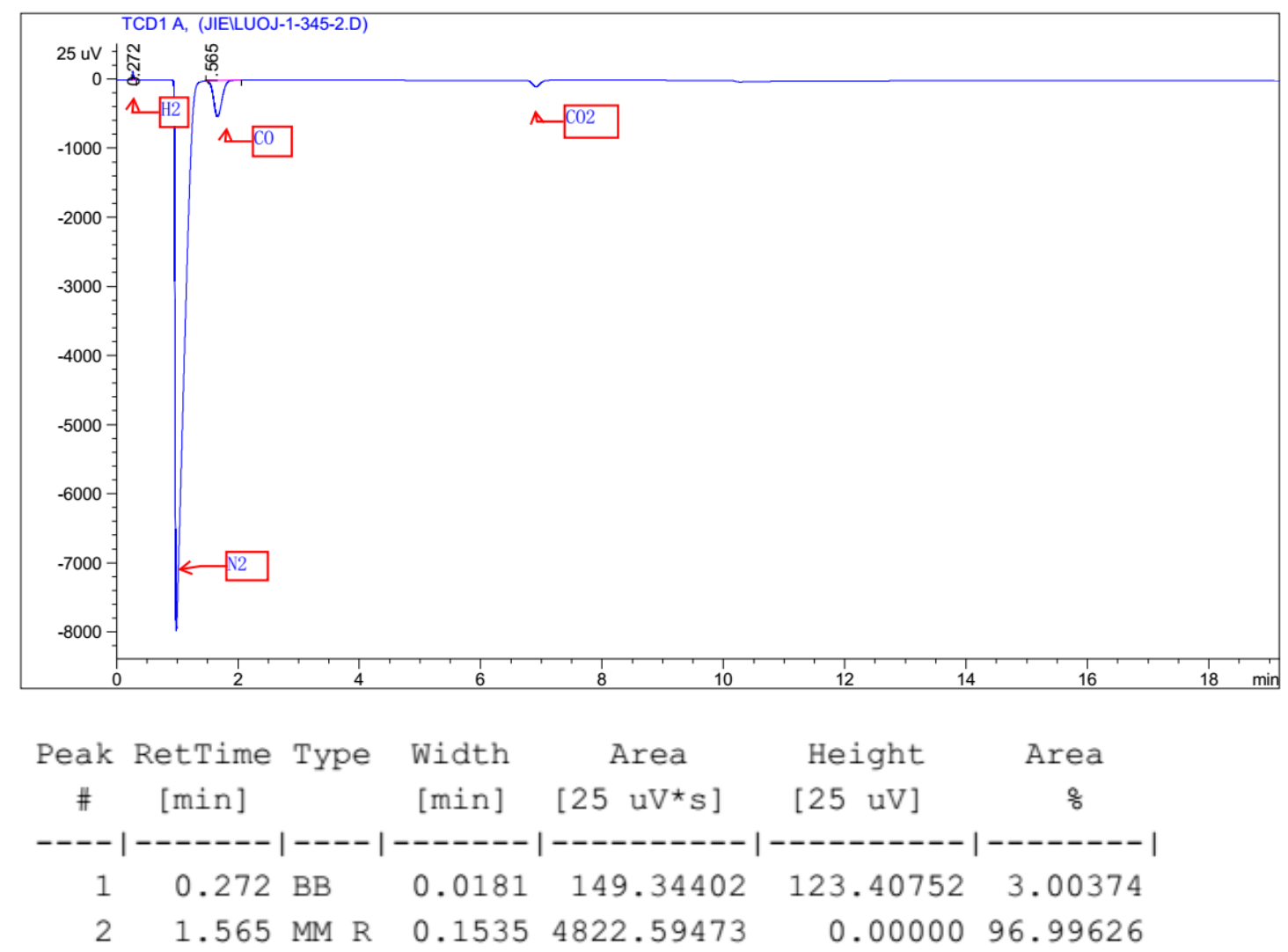

Figure S39. GC trace with $\mathrm{H}_{2}: \mathrm{CO}_{2}=1(0.6): 1$, the amount of produced gas was too little which accounts for the deviation error.

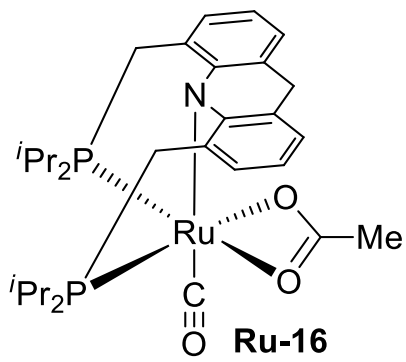

Ru-16: ${ }^{1} \mathrm{H}$ NMR (400 MHz, $\left.\mathrm{C}_{6} \mathrm{D}_{6}\right) \delta 7.24-7.11(\mathrm{~m}, 2 \mathrm{H}$, aryl), $7.05-6.94$ (m, 4H, aryl), 4.10 (d, $\left.J=16.0 \mathrm{~Hz}, 1 \mathrm{H}, \operatorname{ArCH}_{2} \mathrm{Ar}\right), 3.90$ (d, $\left.J=16.2 \mathrm{~Hz}, 1 \mathrm{H}, \operatorname{ArC} \boldsymbol{H}_{2} \mathrm{Ar}\right), 2.80$ $\left(\mathrm{t}, J=10.8 \mathrm{~Hz}, 2 \mathrm{H}, \mathrm{CH}_{2} \mathrm{P}\right), 2.55-2.39\left(\mathrm{~m}, 2 \mathrm{H}, \mathrm{CH}_{2} \mathrm{P}\right), 2.04-1.85(\mathrm{~m}, 4 \mathrm{H}$, $\left.\mathrm{PCH}\left(\mathrm{CH}_{3}\right)_{2},\right), 1.55\left(\mathrm{~s}, 3 \mathrm{H}, \mathrm{COCH}_{3}\right), 1.47-1.33\left(\mathrm{~m}, 6 \mathrm{H}, \mathrm{PCH}\left(\mathrm{CH}_{3}\right)_{2}\right), 1.17-0.93(\mathrm{~m}$, $\left.18 \mathrm{H}, \mathrm{PCH}\left(\mathrm{CH}_{3}\right)_{2}\right) .{ }^{13} \mathrm{C}$ NMR (101 MHz, $\left.\mathrm{C}_{6} \mathrm{D}_{6}\right) \delta 203.06(\mathrm{t}, J=15.6 \mathrm{~Hz}, \mathrm{Ru}-\mathrm{CO})$, $189.86\left(\mathrm{~s}, \mathrm{COCH}_{3}\right), 153.81$ (s, Ar), 129.80 (s, Ar), 127.61 (s, Ar), 126.46 (s, Ar), 122.36 (s, Ar), 118.57 (s, Ar), 34.88 (s, $\mathrm{ArC} \mathrm{H}_{2} \mathrm{Ar}$ ), 29.67 (p, J=8.9 Hz, $\mathrm{PCH}\left(\mathrm{CH}_{3}\right)_{2}$ ), 28.43 (dt, $\left.J=18.6,9.2 \mathrm{~Hz}, \mathrm{CH}_{2} \mathrm{P}\right), 26.16\left(\mathrm{dt}, J=21.6,8.6 \mathrm{~Hz}, \mathrm{PCH}\left(\mathrm{CH}_{3}\right)_{2}\right), 24.11$ (s, 
$\left.\mathrm{COCH}_{3}\right), 20.55$ (s, $\left.\mathrm{PCH}\left(\boldsymbol{C H}_{3}\right)_{2}\right), 19.72$ (s, $\left.\mathrm{PCH}\left(\boldsymbol{C H}_{3}\right)_{2}\right), 19.09$ (s, $\left.\mathrm{PCH}\left(\boldsymbol{C H}_{3}\right)_{2}\right), 18.91$ (s, PCH $\left.\left(\mathrm{CH}_{3}\right)_{2}\right) .{ }^{31} \mathrm{P}$ NMR $\left(162 \mathrm{MHz}, \mathrm{C}_{6} \mathrm{D}_{6}\right) \delta 86.76$.
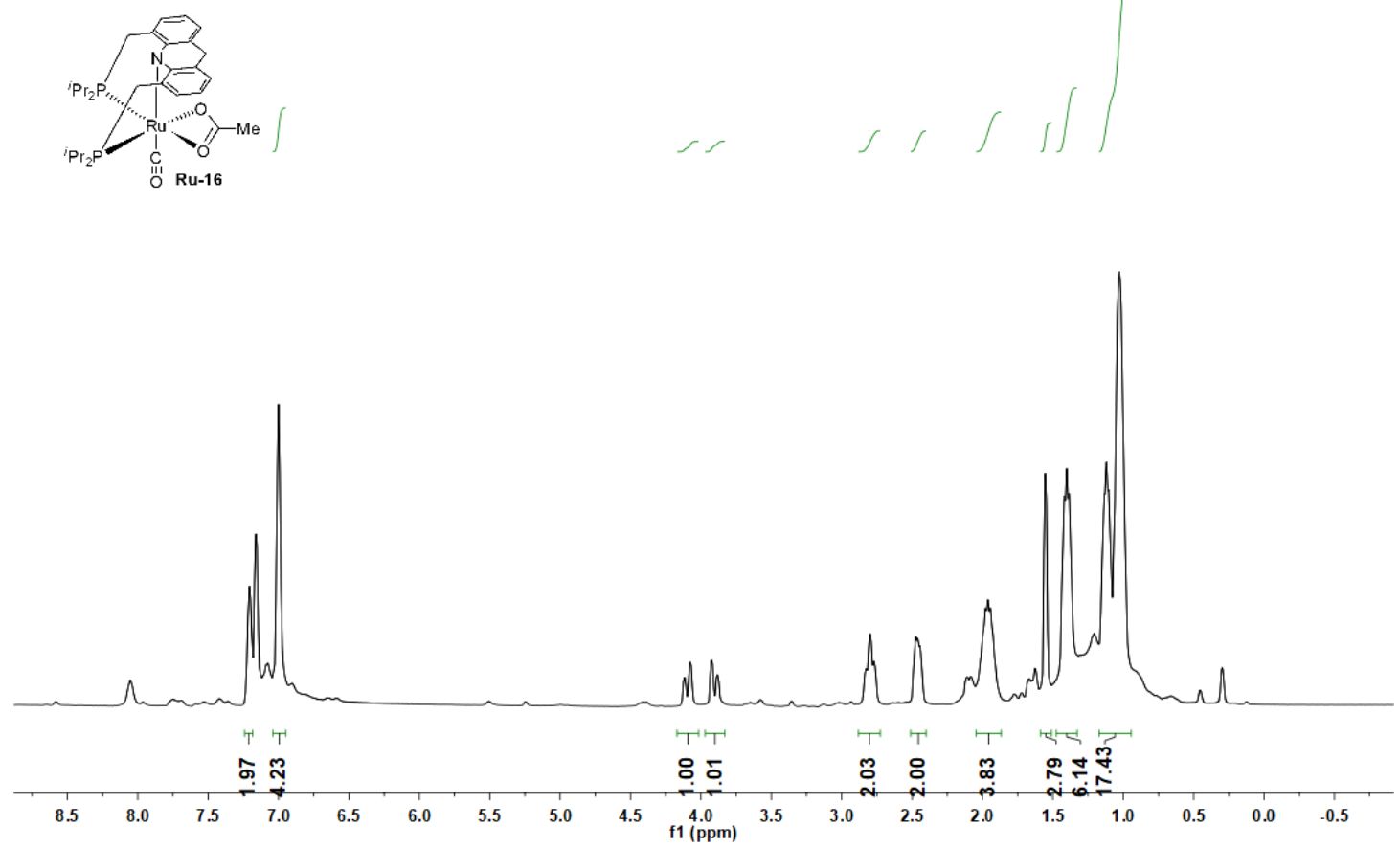

Figure S40. ${ }^{1} \mathrm{H}$ NMR of crude Ru-16 in benzene- $d_{6}$.
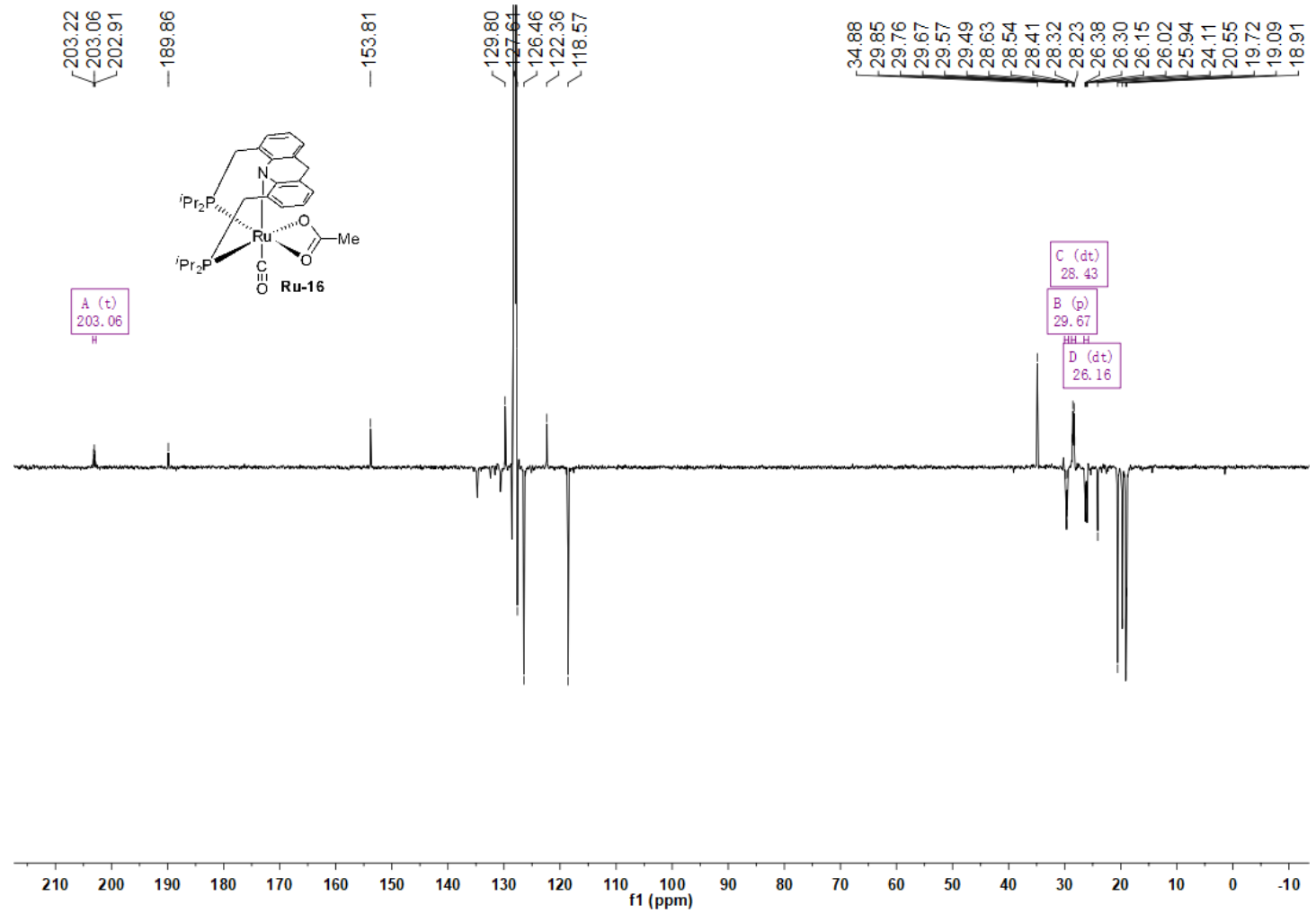

Figure S41. ${ }^{13} \mathrm{C}$ NMR of crude Ru-16 in benzene- $d_{6}$. 


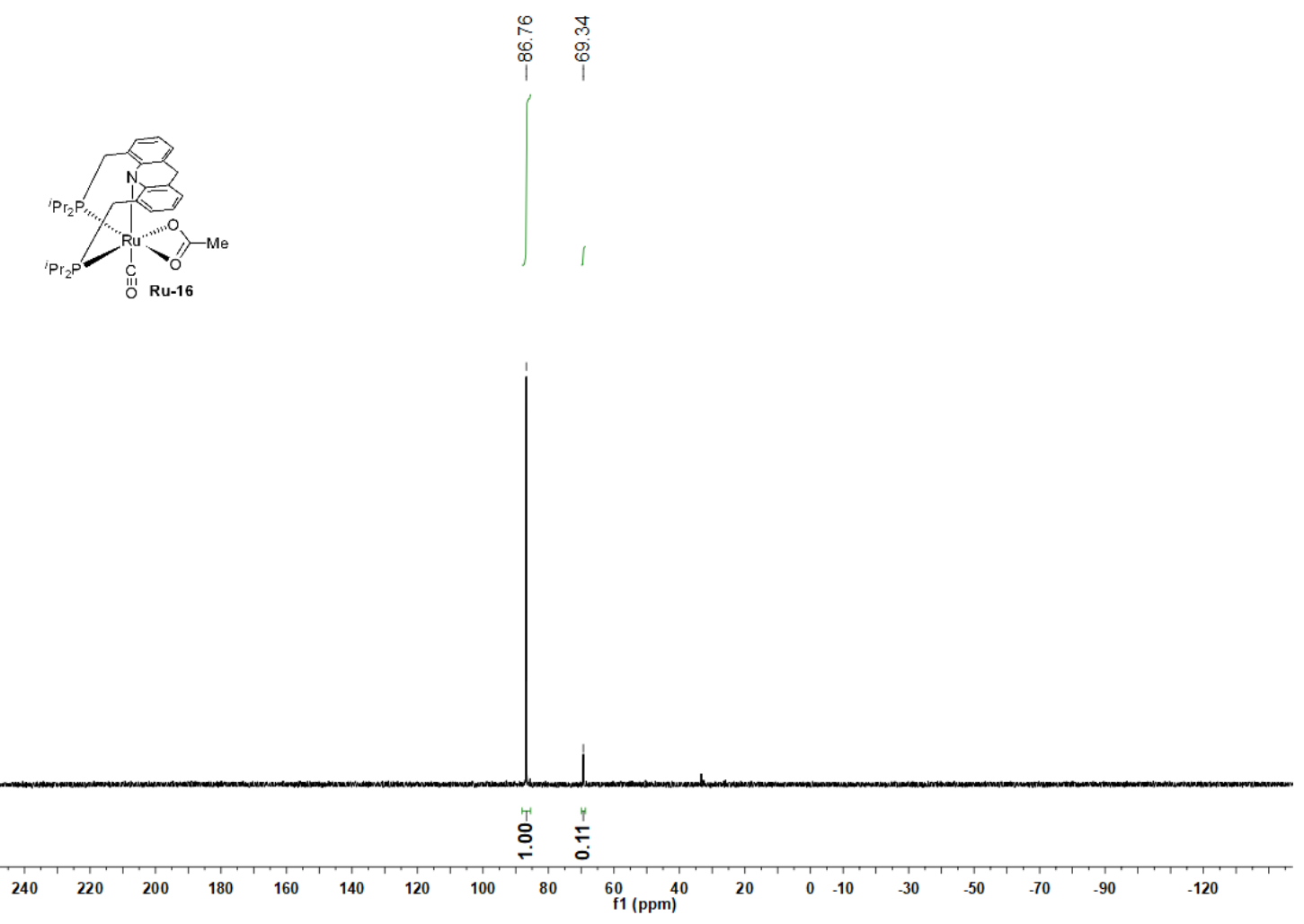

Figure S42. ${ }^{31} \mathrm{P}$ NMR of crude Ru-16 in benzene- $d_{6}$. 


\section{Computational details}

DFT calculations were performed with Gaussian 16 (C.01 revision) ${ }^{12}$ using Truhlar's M06-L functional, ${ }^{13}$ the triple- $\xi$ def2-TZVP basis set, ${ }^{14}$ W06 density fitting, ${ }^{15}$ and Grimme's D3(0) empirical dispersion correction. ${ }^{16}$ Frequency calculations at this level of theory were run at $383.15 \mathrm{~K}$ (experimentally determined initial internal reaction temperature) to confirm stationary points and transition states and to obtain thermodynamic corrections. Single point energies of the M06-L optimized structures were computed with ORCA $(4.2 .1)^{17}$ using the range-separated meta-GGA hybrid functional $\omega \mathrm{B} 97 \mathrm{M}-\mathrm{V}$ of the Head-Gordon group $^{18}$ including dispersion correction, ${ }^{19,20}$ together with the triple- $\xi$ def2-TZVPP basis set $^{14}$ and the corresponding auxiliary basis sets, $\operatorname{def} 2 / \mathrm{J}^{15}$ and def2-TZVPP/C ${ }^{21}$ for RIJCOSX density fitting. The functional and basis set selections are based on recent benchmark studies. $^{22}$ The polarizable continuum model (IEFPCM) was used in all calculations (optimization and single point) with the SMD solvation (Methanol) model of Truhlar and co-workers. $^{23}$

Gibbs free energies were computed by adding the free energy correction term from the frequency calculation to the single point energy in methanol, according to

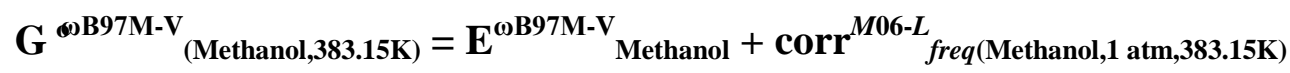

where $\mathbf{E}^{\mathbf{\omega B} \mathbf{B} 7 \mathbf{M}-\mathbf{v}}$ Methanol is the single point energy; and where corr ${ }_{\text {freq }}^{\text {M0 }}$ is the thermal correction to the Gibbs free energy from the frequency calculation (at $\mathrm{T}=383.15 \mathrm{~K}$ and $\mathrm{P}=1 \mathrm{~atm}$ ).

Free energy values $\left(\mathrm{G}^{\mathrm{o}}\right)$ were then corrected to account for changes in standard states $\left(\mathrm{G}^{\mathrm{o}} \rightarrow \mathrm{G}\right)$.

Standard state corrections ${ }^{24}$ were employed such that all species are treated as $1 \mathrm{M}$ (using an ideal gas approximation), with the exception of $\mathrm{H}_{2}, \mathrm{CO}_{2}, \mathrm{CO}$ (maintained as $1 \mathrm{~atm})$, water $(1 \mathrm{~atm}$ to $5.5 \mathrm{M})$ and methanol $(1 \mathrm{~atm}$ to $22 \mathrm{M}) .{ }^{25-27}$ Other than these standard state corrections, the transformation of hydrogen, $\mathrm{CO}_{2}$ and $\mathrm{CO}$ from the condensed phase to the gas phase is not additionally corrected in the free energy quantities provided. 
Ethanethiol were studied as minimal models for hexanethiol in the system. Directionality of $\Delta \mathrm{G}$ and $\Delta \mathrm{G}_{\mathrm{TS}}$ values are indicated by the ordering of $\mathrm{X}, \mathrm{Y}$ and all energies are reported in $\mathrm{kcal} / \mathrm{mol}$.

Table S2 Energy data

\begin{tabular}{|c|c|c|c|c|}
\hline Structure & $\mathbf{E}^{\omega \mathrm{BB} 97 \mathrm{M}-\mathrm{V}}{ }_{\text {Methanol }}$ & $\mathbf{G}^{0 \omega \mathrm{B} 97 \mathrm{M}-\mathrm{V}}{ }_{\text {Methanol }}$ & $\begin{array}{l}\text { Imaginary } \\
\text { Frequency }\end{array}$ & $\begin{array}{c}\mathrm{G} \\
T=383.15 \mathrm{~K}\end{array}$ \\
\hline Energy Unit & Hartree & Hartree & $\mathrm{cm}^{-1}$ & kcal \\
\hline mer Ru-1 & -1999.28346 & -1998.751253 & - & -1254213.976 \\
\hline fac $\mathbf{R u}-1$ & -1999.270686 & -1998.734105 & - & -1254203.216 \\
\hline$f a c$ Ru-2 & -2477.298617 & -2476.693754 & - & -1554122.895 \\
\hline Ru-3 & -2476.110273 & -2475.521106 & - & -1553387.059 \\
\hline Ru-4 & -2112.668395 & -2112.129625 & - & -1325358.904 \\
\hline Ru-5 & -2115.024487 & -2114.439602 & - & -1326808.415 \\
\hline Ru-6 & -2113.816693 & -2113.262898 & - & -1326070.033 \\
\hline Ru-7 & -2113.817184 & -2113.252456 & - & -1326063.48 \\
\hline Ru-8 & -2075.73643 & -2075.17787 & - & -1302171.678 \\
\hline Ru-9 & -2074.538698 & -2073.997816 & - & -1301431.194 \\
\hline Ru-10 & -2189.090472 & -2188.518229 & - & -1373292.753 \\
\hline Ru-11 & -2189.100084 & -2188.53336 & - & -1373302.248 \\
\hline Ru-12 & -2187.938354 & -2187.388297 & - & -1372583.721 \\
\hline Ru-13 & -2187.913267 & -2187.364281 & - & -1372568.651 \\
\hline Ru-15 & -2190.277381 & -2189.690626 & - & -1374028.433 \\
\hline $\mathbf{T S}_{2,3}$ & -2477.277135 & -2476.674399 & -799.9086 & -1554110.75 \\
\hline $\mathbf{T S}_{\text {III }}$ & -2591.814004 & -2591.183875 & -432.5953 & -1625965.446 \\
\hline $\mathbf{T S}_{\mathbf{I V}}$ & -2667.079178 & -2666.445477 & -784.5237 & -1673192.102 \\
\hline $\mathbf{T S}_{5,6}$ & -2114.984669 & -2114.404952 & -1283.1892 & -1326786.672 \\
\hline $\mathbf{T S}_{6,7}$ & -2113.817771 & -2113.25254 & -409.6517 & -1326063.533 \\
\hline $\mathbf{T S}_{\mathbf{8 , 9}}$ & -2075.701105 & -2075.146419 & -1149.2154 & -1302151.942 \\
\hline $\mathbf{T S}_{\mathbf{9 , 1 0}}$ & -2190.236516 & -2189.654216 & -124.1641 & -1374005.585 \\
\hline $\mathbf{T S}_{10,11}$ & -2189.081706 & -2188.517223 & -608.7270 & -1373292.122 \\
\hline $\mathbf{T S}_{11,12}$ & -2189.092849 & -2188.530098 & -1206.7222 & -1373300.201 \\
\hline $\mathbf{T S}_{13,14}$ & -2187.897814 & -2187.354255 & -344.4940 & -1372562.36 \\
\hline $\mathbf{T S}_{15,10}$ & -2190.236516 & -2189.654216 & -1422.1539 & -1374005.585 \\
\hline $\mathbf{T S}_{1,10}$ & -2190.240271 & -2189.654652 & -1127.4550 & -1374005.859 \\
\hline $\mathrm{H}_{2} \mathrm{O}$ & -76.44592285 & -76.44951685 & - & -47968.33838 \\
\hline EtSH & -477.9872272 & -477.9478982 & - & -299909.8707 \\
\hline МeOH & -115.7259917 & -115.7053527 & - & -72600.31985 \\
\hline
\end{tabular}




\begin{tabular}{|c|c|c|c|c|}
\hline $\mathbf{H C H O}$ & -114.5117968 & -114.5135018 & - & -71854.78694 \\
\hline $\mathbf{H O C H}_{2} \mathbf{O H}$ & -190.9818141 & -190.9586491 & - & -119824.1168 \\
\hline $\mathbf{H C O O H}$ & -189.7986995 & -189.7975065 & - & -119095.4999 \\
\hline hydrogen & -1.161258141 & -1.167228141 & - & -732.4356587 \\
\hline $\mathbf{C O}_{2}$ & -188.6177062 & -188.6345492 & - & -118368.1796 \\
\hline $\mathbf{C O}$ & -113.3293545 & -113.3499765 & - & -71127.11022 \\
\hline
\end{tabular}




\section{Cartesian Coordinates}

\section{mer Ru-1}

$\mathrm{Ru}-0.24022500006 .30793300001 .3527310000$

P -1.8503570000 7.5097680000 0.1735700000

P 1.67941100005 .96739400002 .6327950000

O -2.1059200000 5.4151630000 3.4840140000

N $0.97291400006 .1220660000-0.5080690000$

C - 1.36520900005 .82900900002 .6716980000

C -1.6613860000 10.10166700001 .2548310000

C -3.2252170000 8.51347100002 .3913000000

C -2.6226520000 8.94046100001 .0621340000

C $-4.06509300007 .4529080000-1.6174970000$

C - $2.98768900005 .2532640000-1.0412400000$

C -3.3251420000 $6.6563100000-0.5560630000$

C 3.49979500004 .19288700003 .9178090000

C 1.15780300003 .35094600003 .4910580000

C 2.2834480000 4.2555210000 3.0106000000

C 0.73357100006 .60265000005 .2007550000

C 1.90842700008 .42884800003 .9689300000

C 1.83755400006 .92892300004 .2083560000

C $0.37183500006 .1848550000-1.7768850000$

C $2.20947100005 .4527690000-0.4972480000$

C $-0.91157300008 .2981100000-1.1897450000$

C $-0.46273600007 .2622800000-2.1633540000$

C - $0.94243000007 .3316400000-3.4685640000$

C $-0.65533500006 .3623060000-4.4154240000$

C $0.10819400005 .2721520000-4.0286960000$

C $0.59747000005 .1726110000-2.7361210000$

C $1.34679100003 .9728660000-2.2692790000$

C $2.48594500004 .4209940000-1.4206000000$

C $3.73740100003 .8303700000-1.4989420000$

C $4.75456600004 .2072570000-0.6360330000$

C 4.47888900005 .16491300000 .3263120000

C 3.23706500005 .78749500000 .4190970000

C 2.98963800006 .69852500001 .5693840000

H - 0.77731600009 .80095400001 .8210110000

H - 1.326318000010 .53145400000 .3122610000

H -2.1511930000 10.89676300001 .8194810000

H -3.8403530000 9.31684600002 .7997460000

H -3.8563020000 7.62804700002 .3077450000

H - 2.44313100008 .29752900003 .1216660000

H -3.4307650000 9.27300000000 .4029030000

H -4.9943280000 $6.9479810000-1.8881740000$

H -4.3281190000 $8.4576040000-1.2875670000$ 
H -3.4708090000 7.5443470000 -2.5272380000

H -2.4113520000 5.2756040000 -1.9669470000

H -2.4101840000 $4.6956570000-0.3005530000$

H -3.9017290000 $4.6918940000-1.2422880000$

H -3.9757500000 6.55541900000 .3213190000

H 3.91324800003 .18258600003 .9249180000

H 4.29392700004 .86819500003 .5969050000

H 3.24519900004 .44226100004 .9490630000

H 0.78933700003 .63616600004 .4769250000

H 0.31030600003 .36384800002 .8042870000

H 1.50949600002 .32044000003 .5644460000

H 2.57836400003 .90223600002 .0162290000

H - 0.22840700006 .98123000004 .8513350000

H 0.6244890000 5.53463300005 .3847820000

H 0.93978600007 .08113900006 .1597260000

H 2.02597300008 .94551800004 .9230490000

H 2.74693000008 .71790500003 .3373550000

H 0.99331200008 .80610400003 .5085610000

H 2.79735300006 .61129000004 .6271340000

H - $0.06857600008 .7939580000-0.6991530000$

H - $1.50638200009 .0659300000-1.6887570000$

H - $1.55874300008 .1825460000-3.7376320000$

H - $1.03297500006 .4485590000-5.4262170000$

H $0.32172800004 .4720930000-4.7296740000$

H $0.67392700003 .3452180000-1.6630750000$

H $1.67902300003 .3520060000-3.1014890000$

H $3.90118900003 .0525760000-2.2373310000$

H $5.73373900003 .7495600000-0.6952100000$

H 5.24408500005 .44973600001 .0404290000

H 3.90927300006 .87325400002 .1313370000

H 2.5953340000 7.66940300001 .2574860000

H - 0.11240100007 .71152900002 .0383090000

fac Ru-1

H 0.71072000008 .60061000005 .3605640000

P -2.3474790000 7.70881300002 .8148510000

P 0.50258300006 .13199600004 .9548940000

O -2.3245440000 7.7660620000 6.8554630000

N 0.75109200008 .34809900002 .7129560000

C - 1.57676800007 .84428500001 .1507390000

H - 0.92017400006 .97370100001 .0649970000

H - 2.34050700007 .76413400000 .3745970000

C -0.78601700009 .09871600000 .9931630000$ 
C -1.158583000010 .05624900000 .0554960000$

H -2.0558360000 $9.8857300000-0.5306330000$

C $-0.387820000011 .1881910000-0.1675780000$

H -0.6844640000 $11.9142630000-0.9138220000$

C 0.775827000011 .36886300000 .5695420000

H 1.395933000012 .24361600000 .4025280000

C 1.152707000010 .45519700001 .5419060000

C 0.37070400009 .30640400001 .7787610000

C 2.3599520000 10.65290800002 .4069360000

H 3.078626000011 .32722400001 .9381740000

H 2.050134000011 .15204100003 .3382390000

C 2.98614300009 .33909600002 .7610220000

C 4.34696400009 .18441600002 .9798170000

H 5.005768000010 .03193800002 .8201380000

C 4.86323200007 .97554700003 .4304310000

H 5.92507500007 .86546100003 .6105960000

C 4.00090500006 .90968800003 .6511520000

H 4.39148000005 .95869000003 .9981580000

C 2.63696600007 .02355400003 .4071600000

C 2.11252000008 .25508500002 .9602760000

C 1.72026500005 .86254300003 .6023760000

H 2.28850300004 .95419300003 .8046010000

H 1.11640800005 .68619500002 .7066780000

C -3.4785280000 6.25161200002 .6492890000

H - 4.29458300006 .58657600002 .0035610000

C -4.05957500005 .87081900004 .0017820000$

H -3.2825550000 5.55442600004 .6995260000

H - 4.76279000005 .04317100003 .8943880000

H -4.5955740000 6.6990340000 4.4656620000

C - 2.79787600005 .08131500001 .9527070000

H - 2.62255100005 .28377400000 .8972660000

H -3.4296560000 4.19366200002 .0142360000

H - 1.83524500004 .82452400002 .3975280000

C -3.4505270000 9.2086390000 2.8307260000

H - 2.77395100009 .97793900002 .4412900000

C - 3.86696700009 .63816400004 .2287940000

H - 4.45981500008 .87720000004 .7378800000

H -4.4784830000 10.54082300004 .1744910000

H -3.0050940000 9.86353800004 .8568090000

C - 4.64415400009 .11885000001 .8945180000

H -4.3741900000 8.77077200000 .8969820000

H -5.1042570000 10.10225700001 .7827000000

H -5.4113120000 8.44932800002 .2867080000

C 1.59246600006 .17965700006 .4548550000 
H 2.33022400006 .92758400006 .1397000000

C 0.92127400006 .70655100007 .7130290000

H 0.48179800007 .69042200007 .5591530000

H 1.66316600006 .79979000008 .5080160000

H 0.14091600006 .03980700008 .0785610000

C 2.33158200004 .87774800006 .7301280000

H 1.66582300004 .10419100007 .1123940000

H 3.09280600005 .04894500007 .4933650000

H 2.8394210000 4.48132400005 .8518720000

C - 0.51859900004 .58504700005 .0024900000

H - 1.29775100004 .82462200004 .2760620000

C - 1.20495700004 .36595700006 .3425880000

H - 0.50250300004 .04266500007 .1107130000

H -1.9551050000 3.57951100006 .2424940000

H -1.7142400000 5.2570000000 6.7072030000

C 0.18110300003 .32200500004 .5216450000

H 0.55605700003 .41511600003 .5036450000

H - 0.53207300002 .49536300004 .5278930000

H 1.01304700003 .03440000005 .1630510000

C - 1.62188400007 .85988200005 .9225670000

Ru - 0.54717200008 .03596300004 .4688210000

fac Ru-2

S - 0.853306000010 .64432500004 .4173870000

H - 0.618526000010 .96966300003 .1286660000

H 0.84807500008 .52363200005 .3362750000

P -2.3977280000 7.8242220000 2.9837300000

P 0.50887700006 .13850200004 .8680230000

O -2.2436750000 7.8760680000 6.9201090000

N 0.79061500008 .34933700002 .6314720000

C - 1.64998900007 .76040500001 .3022000000

H - 1.00800500006 .87393900001 .3077650000

H - 2.42410800007 .60904100000 .5495400000

C -0.85312300008 .98163900000 .9826160000$

C -1.30356800009 .87079400000 .0128520000$

H -2.2505550000 $9.6674080000-0.4761090000$

C $-0.553660000010 .9763460000-0.3608780000$

H - $0.914220000011 .6518830000-1.1260520000$

C 0.675045000011 .18958500000 .2492160000

H $1.282584000012 .0427200000-0.0363330000$

C 1.143489000010 .34072400001 .2421120000

C 0.37622700009 .22451900001 .6434670000

C 2.437589000010 .62005100001 .9482400000 
H 3.154723000011 .08057400001 .2635660000 H 2.2738100000 11.38173800002 .7232990000 C 3.01891800009 .38638200002 .5700190000 C 4.37132800009 .29439300002 .8729720000 H 5.019018000010 .13049700002 .6282940000 C 4.89668600008 .16838500003 .4922960000 H 5.94982200008 .11668000003 .7379040000 C 4.05583100007 .09712900003 .7660770000 H 4.45510300006 .18907400004 .2068970000 C 2.7062090000 7.1457650000 3.4435190000 C 2.15424800008 .32281600002 .8863410000 C 1.82881800005 .95481700003 .6048920000 H 2.41231700005 .06195600003 .8377630000 H 1.29545300005 .77100600002 .6689980000 C -3.4760180000 6.3116860000 3.0910900000 H - 2.78525000005 .56105400003 .4721370000 C - 4.02122600005 .78420000001 .7733820000 H -4.6816610000 6.49289600001 .2751540000 H -4.6017510000 4.8780870000 1.9601020000 H -3.2274740000 5.51816100001 .0761690000 C -4.57098100006 .46754100004 .1360900000$ H -4.2012970000 6.89613000005 .0688040000 H -4.9961880000 5.49010000004 .3716420000 H -5.3865680000 7.0955150000 3.7780620000 C -3.52358300009 .29898600002 .8335410000$ H - 2.803696000010 .07085100002 .5314000000 C -4.14980900009 .74300200004 .1501980000$ H -5.0388230000 9.1612810000 4.3879570000 H - 4.460699000010 .78696100004 .0800570000 H -3.4670510000 9.6628990000 4.9950650000 C - 4.57738600009 .21765200001 .7393930000 H - 4.16839100008 .92100600000 .7743090000 H -5.0464850000 10.19484500001 .6057550000 H -5.3702480000 8.51643500002 .0003350000 C 1.50010200006 .06789700006 .4419760000 H 2.31256800006 .76451000006 .2006790000 C 0.79367700006 .60016600007 .6772560000 H 0.48549000007 .63707400007 .5587580000 H 1.47044200006 .55572800008 .5325190000 H - 0.08757200006 .01150800007 .9346690000 C 2.11185300004 .70349200006 .7211070000 H 1.36459700003 .99465400007 .0810010000 H 2.86778800004 .79431900007 .5028250000 H 2.5986240000 4.2652240000 5.8499240000 
C -0.35190500004 .50215900004 .7658780000$

H 0.36988200003 .77113500005 .1376890000

C - 0.66732300004 .13523500003 .3184910000

H - 0.98799800004 .99113000002 .7219000000

H - 1.46374000003 .39011700003 .2754610000

H 0.20296400003 .71165000002 .8193850000

C - 1.56126200004 .47421900005 .6881990000

H - 1.27095600004 .58383900006 .7334220000

H -2.0930210000 3.52581000005 .5931160000

H -2.2690090000 5.27364700005 .4664400000

C 0.660550000011 .40811500005 .0935360000

H 0.592682000011 .22744900006 .1662600000

H 1.514591000010 .84170600004 .7270500000

C 0.753621000012 .87604400004 .7713470000

H - 0.091222000013 .43250800005 .1757670000

H 0.783878000013 .04815200003 .6944160000

H 1.665865000013 .29311600005 .1987230000

C - 1.56236400007 .98484400005 .9748530000

Ru -0.52582800008 .16194500004 .5022760000$

\section{Ru-3}

S -0.5159970000 11.03486000003 .4845230000

P - 2.10491400008 .01849600002 .9328650000

P 0.69244500006 .66823900004 .8648570000

O - 1.83444200009 .00493300006 .6670080000

N 0.89808900008 .40853600002 .2747860000

C - 1.58545200007 .88105300001 .1784000000

H - 1.01367300006 .94829700001 .1365060000

H - 2.47954900007 .74723900000 .5693300000

C -0.76021100009 .00625900000 .6577310000$

C - $1.19133600009 .8089100000-0.3902310000$

H - $2.18385200009 .6514640000-0.8000150000$

C $-0.354585000010 .7738090000-0.9380720000$

H -0.6950520000 $11.3867810000-1.7630710000$

C $0.928927000010 .9326690000-0.4289570000$

H $1.592927000011 .6782210000-0.8546360000$

C 1.371449000010 .16990300000 .6426940000

C 0.52077100009 .20625200001 .2079260000

C 2.706424000010 .36794400001 .2943300000

H 3.431523000010 .79338800000 .5977700000

H 2.595710000011 .11252900002 .0962760000

C 3.21333800009 .08689500001 .8877280000

C 4.56502300008 .79111200001 .9754500000 
H 5.28403600009 .49546000001 .5696010000

C 5.00456500007 .62451300002 .5899350000

H 6.06278900007 .40801300002 .6631160000

C 4.07010700006 .73325800003 .0978190000

H 4.39766300005 .80762000003 .5592810000

C 2.70564700006 .98182500002 .9944180000

C 2.2604310000 8.1841170000 2.4033830000

C 1.70961800005 .98711800003 .4911660000

H 2.2138730000 5.07593600003 .8124300000

H 0.99645600005 .71671500002 .7055000000

C - 2.76514500006 .32552400003 .2766470000

H - 1.82592400005 .76376500003 .3199760000

C -3.60637100005 .66700900002 .1898430000$

H -4.6070320000 6.0865680000 2.1242110000

H -3.7189410000 4.60893100002 .4334940000

H -3.1483360000 5.72049500001 .2041980000

C - 3.45422600006 .24326400004 .6290570000

H -2.8781280000 6.71455100005 .4256690000

H -3.6142220000 5.2006940000 4.9102780000

H -4.4333060000 6.72293200004 .5972640000

C - 3.49584800009 .24054100002 .8399490000

H -3.0122390000 10.04978200002 .2798780000

C -3.9315460000 9.80411300004 .1797830000

H -4.3273870000 9.0345510000 4.8434320000

H -4.7246420000 10.53798600004 .0260800000

H -3.1157210000 10.30791000004 .6890650000

C -4.70404100008 .75695800002 .0507390000$

H -4.4492690000 8.30056200001 .0953280000

H -5.3507980000 9.6099490000 1.8381040000

H -5.2962780000 8.04297800002 .6230580000

C 2.02104500007 .18349600006 .0652950000

H 2.6573910000 7.7899890000 5.4098690000

C 1.54548900008 .09535400007 .1855230000

H 1.09186000009 .00787500006 .8009620000

H 2.3977940000 8.39243400007 .7993680000

H 0.82379000007 .61608900007 .8458540000

C 2.87213300006 .04334500006 .6045420000

H 2.3300120000 5.4498900000 7.3410560000

H 3.75201600006 .45160400007 .1057920000

H 3.22635800005 .37062800005 .8242360000

C -0.22000500005 .21589300005 .5804360000$

H -1.1039270000 5.1478290000 4.9459960000

C - -0.70331400005 .49494900006 .9962230000$

H 0.11356900005 .45005200007 .7165810000 
H - 1.43537100004 .74034000007 .2890900000

H - 1.18282700006 .46882500007 .0930370000

C 0.48837300003 .87233900005 .4833690000

H 0.70658700003 .59347700004 .4537630000

H -0.1629080000 3.09844000005 .8941940000

H 1.41901700003 .84234400006 .0468960000

C - 0.909476000011 .98942100004 .9924460000

H - 1.127249000013 .00408000004 .6528600000

H -1.8328690000 11.61495400005 .4381240000

C 0.192269000012 .01042900006 .0243970000

H 1.117068000012 .41537200005 .6116400000

H 0.409515000011 .00489100006 .3881360000

H - 0.085131000012 .62150800006 .8869440000

C - 1.24849700008 .88048200005 .6647720000

$\mathrm{Ru}-0.30078500008 .68383800004 .1218470000$

\section{Ru-4}

Ru 20.25122100000 .25919700006 .4091560000

P $18.4533370000-1.15900700005 .8612470000$

P 21.6757130000 2.03428100005 .8051910000

O $22.3399770000-1.85495800006 .1100900000$

O 20.55476800000 .01382700009 .4619290000

N 18.66720300001 .86853600007 .1113300000

C 21.5248970000 -1.0312070000 6.1552370000

C 20.44714400000 .09848500008 .3180920000

C $19.0302540000-3.28913700007 .5937400000$

C $16.6624760000-3.16509900006 .7574290000$

C $17.9031370000-2.37479900007 .1404190000$

C $18.2155880000-1.18523500003 .0644800000$

C $19.8568550000-2.78746600004 .0440480000$

C $18.5291070000-2.07800700004 .2547370000$

C 23.71515100003 .81997100006 .6335240000

C 23.83520400001 .46442700007 .5035510000

C 22.92176300002 .59265100007 .0513250000

C 21.6474150000 2.2262190000 3.0051710000

C 23.25285000000 .58460300003 .9790610000

C 22.56129400001 .92298600004 .1820770000

C 17.49425300001 .38569200007 .6992150000

C 19.17138900003 .04495100007 .6740730000

C $17.0250850000-0.02350600005 .6558710000$

C 16.62546800000 .53050300006 .9761660000

C 15.40851900000 .13671600007 .5235590000

C 14.99992800000 .55786400008 .7777720000 
C 15.85759100001 .36344800009 .5106970000

C 17.08666700001 .75456300009 .0022400000

C 18.04053600002 .54302700009 .8346710000

C 18.82725100003 .47540900008 .9765030000

C 19.23998800004 .70728300009 .4606900000

C 20.04333200005 .54509100008 .7026830000

C 20.43960100005 .11406400007 .4478410000

C 20.02375400003 .89389100006 .9245540000

C 20.55039700003 .47032200005 .6003070000

H $19.3119640000-3.99836600006 .8145130000$

H $19.9226110000-2.73298300007 .8831390000$

H $18.7108140000-3.87286200008 .4583080000$

H 16.2662120000 -3.6842730000 7.6316990000

H $15.8656050000-2.53548800006 .3622950000$

H 16.8895860000 -3.9257390000 6.0094250000

H 17.6526610000 -1.7120750000 7.9779900000

H $18.3089730000-1.76232800002 .1432500000$

H $17.2065680000-0.77862600003 .0940830000$

H $18.9161380000-0.35035300002 .9960590000$

H $20.6502990000-2.06712500003 .8373990000$

H $20.1643580000-3.38984000004 .8973580000$

H $19.7895860000-3.44986300003 .1796720000$

H $17.7359030000-2.82734600004 .3390030000$

H 24.4564610000 3.57507500005 .8718450000

H 23.0845320000 4.6176300000 6.2416940000

H 24.2577270000 4.2228750000 7.4903760000

H 24.4388370000 1.79171500008 .3513540000

H 23.2774590000 0.5815000000 7.8176370000

H 24.5263150000 1.16471400006 .7148670000

H 22.27823500002 .86212300007 .8979600000

H 20.80364600001 .53409500002 .9659890000

H 21.2526590000 3.24017300003 .0256560000

H 22.20420800002 .11054500002 .0740370000

H 23.89455500000 .62951600003 .0977830000

H 23.8730600000 0.2869060000 4.8230610000

H $22.5202530000-0.20539200003 .8047470000$

H 23.32025800002 .70949100004 .2376370000

H 16.1919340000 - 0.55058500005 .1875220000

H 17.36774000000 .75296600004 .9685240000

H 14.7682730000 - 0.50916800006 .9326880000

H 14.04066200000 .25577300009 .1780380000

H 15.58420100001 .688138000010 .5094470000

H 18.72606700001 .857225000010 .3518310000

H 17.5187020000 3.082694000010 .6269180000 
H 18.93426100004 .998957000010 .4602480000 H 20.3624430000 6.5064660000 9.0842540000 H 21.0824150000 5.73886200006 .8374560000 H 19.75834600003 .12610100004 .9317990000 H 21.0768700000 4.2916560000 5.1107950000 H 19.97574100000 .50826500004 .7748510000

\section{Ru-5}

O -0.7259820000 10.44070900004 .0766560000 H -0.1766320000 10.67749300003 .3141940000 H 1.00425500008 .50156800005 .2481960000 P -2.3038740000 7.78948100002 .9499700000 P 0.58160900006 .08012500004 .8469570000 O -2.0293190000 7.9628670000 6.9265070000 N 0.87924200008 .20828400002 .5778670000 C - 1.59212500007 .70440000001 .2493460000 H - 0.98710600006 .79234700001 .2335600000 H - 2.38470500007 .59440300000 .5084790000 C -0.75497500008 .90117000000 .9357900000$ C - $1.16411300009 .8086380000-0.0352140000$ H - $2.10306900009 .6298150000-0.5486100000$ C $-0.386625000010 .9062610000-0.3780090000$ H - $0.717385000011 .5919510000-1.1476410000$ C 0.821397000011 .10909100000 .2756230000 H 1.439686000011 .96493200000 .0246330000 C 1.247182000010 .24326700001 .2724010000 C 0.46647500009 .11827200001 .6184060000 C 2.496299000010 .49248000002 .0657850000 H 3.206912000011 .10014500001 .5021770000 H 2.238677000011 .09599200002 .9511570000 C 3.11571300009 .20916700002 .5281500000 C 4.47692500009 .07391500002 .7635780000 H 5.13424400009 .90855600002 .5414420000 C 4.99619300007 .90497000003 .3058430000 H 6.05748100007 .81448900003 .4998890000 C 4.13777400006 .85040000003 .5921030000 H 4.5297530000 5.9246570000 4.0013840000 C 2.77689600006 .94320300003 .3309630000 C 2.2452840000 8.14270800002 .8096130000 C 1.85521100005 .79542700003 .5486760000 H 2.40372700004 .88390500003 .7934390000 H 1.28538400005 .60781900002 .6356140000 C -3.4748620000 6.35029800003 .0767770000 
H -2.8300070000 5.54300000003 .4219170000

C -4.1061080000 5.88490300001 .7742320000

H - 4.73237200006 .64618800001 .3115360000

H -4.7416350000 5.0185930000 1.9699000000

H -3.3587640000 5.5756360000 1.0441460000

C - 4.51246000006 .57607800004 .1671770000

H - 4.07011000006 .95325400005 .0908020000

H -5.0098890000 5.6337710000 4.4041400000

H -5.2845080000 7.2791380000 3.8549980000

C -3.3281090000 9.33797300002 .8359770000

H - 2.573622000010 .04685300002 .4795840000

C -3.8239110000 9.8555300000 4.1791290000

H -4.6774410000 9.2888270000 4.5487090000

H - 4.150734000010 .89250400004 .0763630000

H -3.0500740000 9.8319880000 4.9445100000

C -4.45140600009 .30988400001 .8109210000$

H - 4.12496100008 .95625600000 .8333300000

H -4.8495920000 10.31779800001 .6738740000

H -5.2808590000 8.68104500002 .1353110000

C 1.61142800005 .99651000006 .3941370000

H 2.44782500006 .65029000006 .1174910000

C 0.96138900006 .58332300007 .6355570000

H 0.69688800007 .63061000007 .5039860000

H 1.65694600006 .52396800008 .4746460000

H 0.06154900006 .03954000007 .9255890000

C 2.1685610000 4.61107600006 .6860400000

H 1.40032100003 .94462400007 .0808360000

H 2.9491020000 4.68338700007 .4451170000

H 2.61098200004 .13325800005 .8119970000

C -0.36980200004 .49190600004 .8060430000$

H 0.31326700003 .73731500005 .2030700000

C -0.71375700004 .08986000003 .3750640000$

H - 1.00670500004 .93921500002 .7548210000

H - 1.54006300003 .37692800003 .3659500000

H 0.13221900003 .61428300002 .8811170000

C - 1.57859400004 .56330800005 .7259000000

H - 1.28738700004 .71669400006 .7654290000

H -2.1497050000 3.63439400005 .6785850000

H - 2.24981200005 .37894600005 .4552510000

C -0.370531000011 .28480300005 .1702650000$

H - 0.996593000011 .00154600006 .0137230000

H 0.676591000011 .16171100005 .4537570000

C -1.37478800008 .00287300005 .9557350000$

$\mathrm{Ru}-0.37712800008 .08174000004 .4455230000$ 
H - 0.559542000012 .33101000004 .9225240000
Ru-6
$\mathrm{Ru}-0.3364660000-0.18298600000 .5825190000$
P - $1.32237400001 .6428180000-0.2809670000$
P - $0.7434650000-1.9797680000-0.8948940000$
O 0.64894200001 .00367100002 .0215530000
O - $2.7989970000-0.79170200002 .1644360000$
N $1.44325000000 .2141090000-0.6767200000$
C - $0.16452000002 .3216350000-1.5372000000$
H - $0.12825600001 .5699920000-2.3317460000$
H -0.5876610000 $3.2340910000-1.9570980000$
C $1.19683900002 .5765200000-0.9799310000$
C $1.71209100003 .8639930000-0.8837000000$
H $1.10258300004 .6990000000-1.2129210000$
C $2.99618200004 .0865180000-0.4047390000$
H $3.39143700005 .0929440000-0.3502270000$
C 3.76794400003 .00489700000 .0054360000
H 4.76643400003 .16885400000 .3980010000
C $3.26832100001 .7125110000-0.0452010000$
C $1.97666100001 .4820370000-0.5529010000$
C $0.6400380000-2.0470570000-2.1055500000$
H $0.5364370000-2.9391200000-2.7259680000$
H $0.5078510000-1.1731020000-2.7494010000$
C $1.9785130000-1.9981110000-1.4493830000$
C $2.8747570000-3.0564250000-1.5429340000$
H $2.5710450000-3.9542130000-2.0714260000$
C $4.1536740000-2.9602800000-1.0111340000$
H $4.8477070000-3.7858320000-1.1053830000$
C $4.5396550000-1.7869470000-0.3730360000$
H $5.5417970000-1.69505100000 .0332610000$
C $3.6554010000-0.7285330000-0.2267670000$
C $2.3522990000-0.8287370000-0.7515600000$
C 4.00997000000 .53096200000 .5069790000
H 5.08851900000 .70035000000 .5009490000
H 3.73910500000 .41476900001 .5670820000
C - $2.95784300001 .4767970000-1.1313500000$
H -2.8989490000 $0.4643740000-1.5303930000$
C -3.1574630000 $2.4220070000-2.3080380000$
H -4.1240920000 2.2133580000 -2.7699380000
H - $2.39764600002 .2967760000-3.0775670000$
H -3.1626020000 $3.4684760000-2.0047710000$
C -4.1441390000 $1.5185990000-0.1795080000$ 
H -4.3243550000 2.52346200000 .2009600000

H -4.0295850000 0.85078300000 .6725310000

H -5.0436610000 $1.2113160000-0.7155950000$

C - 1.40876400003 .01941000000 .9537460000

H - 0.34483100003 .16319100001 .1724430000

C - 2.09550000002 .67312500002 .2658910000

H - 1.97603700003 .50552900002 .9618130000

H - 1.65908200001 .79363800002 .7344070000

H -3.1650500000 2.5063620000 2.1475340000

C - 1.95143400004 .31955200000 .3741220000

H -3.0240090000 4.2666140000 0.1868180000

H - $1.46187100004 .6116190000-0.5537220000$

H - 1.79096100005 .12627300001 .0916800000

C $-0.3987910000-3.53033000000 .0864510000$

H $0.6804400000-3.43687400000 .2549830000$

C - $1.0639910000-3.59644400001 .4521190000$

H - $2.1513200000-3.54670700001 .3912240000$

H - $0.7279640000-2.79852100002 .1136980000$

H $-0.8109910000-4.54236800001 .9345940000$

C $-0.6380310000-4.8117540000-0.6977220000$

H - $0.1885200000-5.6541420000-0.1693620000$

H - $0.2065440000-4.7884990000-1.6983500000$

H - $1.7033280000-5.0262250000-0.7961160000$

C $-2.2249950000-2.3619660000-1.9325960000$

H - $2.0833590000-3.4020230000-2.2379970000$

C $-3.5040260000-2.2839170000-1.1142470000$

H -3.6680270000 - $1.2918720000-0.6921550000$

H -3.4953230000 - $2.9910440000-0.2845420000$

H $-4.3667760000-2.5221450000-1.7383700000$

C - $2.2577340000-1.5238750000-3.2054100000$

H -3.2273650000 -1.6283690000 -3.6946010000

H - $1.4981110000-1.8477920000-3.9151950000$

H - $2.0966230000-0.4601670000-3.0278550000$

C 0.94790700000 .15553300003 .0770910000

H $1.3185150000-0.83741200002 .7508860000$

C - $1.8307700000-0.54700100001 .5572780000$

H 1.74690100000 .58021400003 .7028640000

H $0.0878950000-0.03942100003 .7445400000$

\section{Ru-7}

$\mathrm{Ru}-0.3357970000-0.31899500000 .7704930000$

P - $1.42242400001 .6656010000-0.2195100000$

P - $-0.6563350000-2.1432460000-0.6247140000$ 
O 0.53431700001 .13167100002 .1866110000

O -2.7331410000 -0.9884770000 2.4192250000

N $1.45938600000 .1908030000-0.4826470000$

C - $0.23584100002 .2484750000-1.4964520000$

H - $0.17932500001 .4388740000-2.2307880000$

H -0.6330110000 $3.1295430000-2.0011410000$

C $1.11393900002 .5424560000-0.9295640000$

C $1.58186400003 .8521780000-0.9048310000$

H $0.93795100004 .6395650000-1.2823580000$

C $2.85483600004 .1568420000-0.4472340000$

H $3.20922600005 .1797570000-0.4498550000$

C 3.67068900003 .12671900000 .0013600000

H 4.67103100003 .34351500000 .3624230000

C 3.22342900001 .81428300000 .0227240000

C $1.93016400001 .4961710000-0.4414240000$

C $0.7725330000-2.1855230000-1.7697140000$

H $0.7162760000-3.1025580000-2.3592160000$

H $0.6121030000-1.3419540000-2.4458580000$

C $2.0997810000-2.0420310000-1.1100260000$

C $3.0296900000-3.0733190000-1.1235100000$

H $2.7516740000-4.0219500000-1.5716520000$

C $4.3091160000-2.8887560000-0.6157770000$

H $5.0309610000-3.6951210000-0.6425750000$

C $4.6560690000-1.6496890000-0.0938940000$

H $5.6571880000-1.48460200000 .2915040000$

C $3.7356850000-0.6117990000-0.0314460000$

C $2.4300970000-0.8017210000-0.5185190000$

C 4.06927800000 .71214200000 .5868600000

H 5.13141300000 .93965700000 .4705050000

H 3.91005900000 .65874800001 .6733820000

C -3.0669010000 $1.5475020000-1.0698010000$

H -3.0478560000 $0.5432690000-1.4909940000$

C - $3.27577400002 .5109770000-2.2277110000$

H $-4.26111200002 .3382380000-2.6650850000$

H - $2.54243700002 .3661100000-3.0201620000$

H -3.2370120000 $3.5550440000-1.9199760000$

C - $4.21931900001 .5888760000-0.0770430000$

H -4.3994000000 2.59846400000 .2914340000

H -4.0527710000 0.94132200000 .7847770000

H -5.1357400000 $1.2543180000-0.5661970000$

C - 1.46441000003 .08732000000 .9678050000

H -0.39760700003 .18761200001 .1934320000$

C - 2.17872100002 .78810100002 .2782120000

H - 1.91449400003 .54600000003 .0179230000 
H - 1.90125500001 .81966500002 .6912270000

H -3.2617860000 2.8129340000 2.1696630000

C - 1.94207200004 .40225000000 .3701800000

H -3.0163960000 4.39456600000 .1844380000

H - $1.43975600004 .6523270000-0.5637390000$

H - 1.74415000005 .21528300001 .0717260000

C - $0.5325910000-3.79105700000 .2204000000$

H $0.5421820000-3.82487100000 .4400240000$

C $-1.2731350000-3.89466500001 .5428250000$

H - $2.3503770000-3.77640500001 .4232520000$

H - $0.9280050000-3.15657000002 .2649960000$

H - $1.1027650000-4.88049600001 .9782280000$

C $-0.8673810000-4.9650880000-0.6862650000$

H -0.5506570000 -5.8936860000 -0.2094820000

H - $0.3692670000-4.9125130000-1.6543390000$

H - $1.9409600000-5.0453660000-0.8617450000$

C - $2.1054080000-2.2668240000-1.7634010000$

H - $2.0494430000-3.2771490000-2.1761820000$

C - $3.4108810000-2.1472420000-0.9922410000$

H -3.4821310000 - $1.2071260000-0.4447850000$

H - $3.5247690000-2.9525430000-0.2663090000$

H - $4.2603560000-2.1975350000-1.6752050000$

C - $1.9794530000-1.2883010000-2.9262860000$

H - $2.9519940000-1.1226930000-3.3926320000$

H - $1.3090540000-1.6700220000-3.6946290000$

H - $1.5919730000-0.3147890000-2.6222810000$

C $0.9522590000-0.04208100002 .5006540000$

H $0.5158420000-1.64407700001 .1969340000$

C - $1.8012500000-0.73460700001 .7692400000$

H $1.9847270000-0.33005400002 .2656330000$

H $0.5241480000-0.55598600003 .3721260000$

\section{Ru-8}

O 1.15965400009 .40983300005 .6041730000

P -2.3535220000 7.8817160000 2.8695240000

P 0.36936600006 .06190100004 .8681670000

O -2.6084620000 7.9904190000 6.6599810000

N 0.71270300008 .36838300002 .6466370000

C - 1.62393400008 .04473500001 .1889340000

H - 1.00139800007 .15529100001 .0673470000

H - 2.42868700007 .99250900000 .4535080000

C - 0.78957700009 .26188500001 .0002310000

C - 1.136039000010 .25787200000 .0966200000 
H -2.0642360000 $10.1622530000-0.4578160000$

C $-0.298097000011 .3432590000-0.1280780000$

H - $0.571232000012 .1059300000-0.8463360000$

C 0.896195000011 .43538600000 .5761170000

H 1.557814000012 .28064700000 .4154470000

C 1.248828000010 .47504500001 .5142440000

C 0.40698500009 .37336000001 .7402590000

C 2.462820000010 .59525400002 .3835990000

H 3.236859000011 .20089500001 .9083450000

H 2.1859550000 11.14126700003 .2985770000

C 2.99202400009 .24654900002 .7708250000

C 4.33712400009 .02806500003 .0283690000

H 5.03636100009 .84719700002 .8947820000

C 4.78827500007 .79279600003 .4756810000

H 5.83835100007 .63374600003 .6854330000

C 3.87522000006 .76150800003 .6433800000

H 4.21583100005 .78589400003 .9741500000

C 2.52360000006 .93341100003 .3621410000

C 2.05785400008 .19942500002 .9329620000

C 1.57425100005 .78875400003 .4996330000

H 2.1229580000 4.85861600003 .6500540000

H 0.96617300005 .67954400002 .5961030000

C -3.2765080000 6.2826400000 2.7326230000

H - 4.11109000006 .49532600002 .0602660000

C -3.8524940000 5.86709800004 .0770310000

H -3.0783560000 5.75039200004 .8355040000

H -4.3762660000 4.9136770000 3.9882330000

H -4.5659150000 6.5996610000 4.4555250000

C -2.4215260000 5.2049920000 2.0719680000

H - 2.41431900005 .31280900000 .9884310000

H - 2.81462500004 .21253800002 .2989320000

H - 1.38026600005 .22812600002 .3990190000

C -3.6572470000 9.2087230000 2.8323230000

H -3.0752660000 10.05262000002 .4417470000

C - 4.20357100009 .61728200004 .1895890000

H - 4.72716200008 .79944600004 .6860760000

H -4.9218760000 10.42944900004 .0642970000

H -3.4209110000 9.9732830000 4.8563550000

C - 4.79382500008 .92637500001 .8605680000

H - 4.45023200008 .59132700000 .8820050000

H -5.3744060000 9.83700200001 .7045990000

H -5.4798560000 8.17390700002 .2520420000

C 1.51110600006 .19220400006 .3314500000

H 2.1887470000 6.98016500005 .9824320000 
C 0.83839500006 .70276000007 .5988620000

H 0.15250100007 .52874500007 .4084080000 H 1.59309800007 .05327500008 .3056850000 H 0.27020200005 .92258100008 .1032140000 C 2.35880800004 .96357100006 .6215810000 H 1.76365300004 .15616300007 .0484340000 H 3.13173600005 .21183200007 .3522900000 H 2.86265600004 .57781600005 .7360210000 C - 0.57271700004 .46772500005 .0329530000 H - 1.39070700004 .59954900004 .3259670000 C - 1.20338400004 .33244300006 .4114570000 H - 0.47026200004 .05492000007 .1686600000 H - 1.96178000003 .54753700006 .3940980000 H -1.6924910000 5.25149300006 .7386320000 C 0.17070400003 .20773300004 .6188380000 H 0.48529200003 .24144600003 .5762760000 H - 0.49130800002 .34590800004 .7268010000 H 1.05194100003 .01603900005 .2294090000 C - 1.86394700008 .08102200005 .7599490000 $\mathrm{Ru}-0.70706900008 .20342300004 .3687060000$ H 1.23429300009 .12570600006 .5238800000 H 1.96781500009 .07484300005 .1872500000 H -1.1640940000 9.74000600003 .9757030000

\section{Ru-9}

O 0.86341300009 .32123200005 .3255290000 P - 2.26233100007 .67718200002 .8681390000 P 0.39237800006 .13626900004 .8566610000 O -2.4410090000 7.7299310000 6.7268320000 N 0.76517500008 .33717300002 .6693710000 C - 1.54742300007 .89303400001 .1860380000 H - 0.88075700007 .03866800001 .0395410000 H - 2.34839300007 .82302900000 .4476800000 C -0.78076700009 .16324800001 .0311590000$ C - 1.171806000010 .14928300000 .1331560000 H - $2.090317000010 .0122950000-0.4283190000$ C $-0.383884000011 .2723120000-0.0826590000$ H - $0.691368000012 .0259380000-0.7965500000$ C 0.813079000011 .40930800000 .6112540000 H 1.444976000012 .27420600000 .4364980000 C 1.210691000010 .46410900001 .5453670000 C 0.40285700009 .33557200001 .7814950000 C 2.465656000010 .60271700002 .3563010000 
H 3.207189000011 .20821900001 .8310080000 H 2.2367220000 11.15874000003 .2768670000 C 3.02714900009 .26269100002 .7335130000 C 4.37830500009 .05247600002 .9635620000 H 5.07281900009 .87218800002 .8094930000 C 4.84515300007 .82338700003 .4168370000 H 5.90089900007 .67437200003 .6047580000 C 3.94494800006 .78619800003 .6221720000 H 4.29919300005 .81834300003 .9610550000 C 2.58933300006 .95470400003 .3657070000 C 2.11501200008 .20924300002 .9293200000 C 1.61866400005 .83092500003 .5204940000 H 2.13941000004 .89527000003 .7241080000 H 1.02868400005 .69581000002 .6089800000 C -3.3448590000 6.1946750000 2.6632980000 H - 4.13228600006 .51900600001 .9784160000 C -4.00327800005 .81196600003 .9796980000$ H -3.2769980000 5.57623200004 .7577860000 H -4.6378140000 4.93459400003 .8448420000 H -4.6330510000 6.6165040000 4.3604340000 C - 2.59598400005 .05298600001 .9814320000 H - 2.51716200005 .21878400000 .9080600000 H -3.1257480000 4.11070000002 .1291600000 H -1.58018700004 .91902500002 .3552290000$ C -3.4163290000 9.1449640000 2.9125520000 H - 2.76513800009 .93425700002 .5194850000 C - 3.86655100009 .58094100004 .2971850000 H - 4.41326800008 .79869700004 .8244430000 H - 4.535668000010 .43889600004 .2093900000 H -3.0295520000 9.88737900004 .9228580000 C - 4.60356700009 .01060200001 .9708680000 H - 4.32263900008 .66906300000 .9746540000 H -5.0921860000 9.9796590000 1.8562190000 H -5.3497480000 8.31908100002 .3652780000 C 1.49268800006 .28037500006 .3453580000 H 2.1885010000 7.0565840000 6.0058150000 C 0.82216400006 .79607900007 .6087360000 H 0.28928600007 .73051200007 .4459480000 H 1.58369100006 .98457500008 .3673490000 H 0.11995300006 .07882300008 .0310500000 C 2.29551900005 .01840100006 .6328170000 H 1.66961800004 .22059100007 .0323690000 H 3.05293900005 .23918300007 .3871180000 H 2.8155600000 4.63227900005 .7575920000 
C - 0.61240500004 .58615800005 .0010640000

H - 1.42739300004 .77758500004 .3046260000

C - 1.23385300004 .39270000006 .3765870000

H - 0.49474200004 .09425100007 .1193170000

H - 1.97623300003 .59452900006 .3237510000

H - 1.74064500005 .28365500006 .7435870000

C 0.09891000003 .32524700004 .5305580000

H 0.41266900003 .38708300003 .4899180000

H - 0.58847300002 .48115800004 .6113220000

H 0.97415000003 .08847700005 .1342540000

C - 1.70809700007 .89342700005 .8329430000

Ru - 0.57544000008 .11905400004 .4213470000

H 0.64477700009 .44036100006 .2557670000

\section{Ru-10}

Ru 10.45266300003 .64998700002 .5158250000

O 11.78095700004 .11257200004 .1398860000

P 9.33955700002 .04110200003 .6793300000

P 9.73521700003 .42456300000 .3028590000

O 8.26033600005 .58845100003 .1248670000

N 11.96526200002 .06031800001 .9913320000

C 10.25198100000 .45688400003 .4774030000

H $9.7635990000-0.31550100004 .0709860000$

H 10.14079500000 .19031300002 .4220010000

C 11.69185200000 .57875700003 .8490800000

C $12.2250850000-0.11431200004 .9294000000$

H $11.5725760000-0.74880200005 .5199700000$

C $13.5764810000-0.02845300005 .2360900000$

H $13.9837830000-0.58643900006 .0696820000$

C 14.40077100000 .78073400004 .4617910000

H 15.45584800000 .86643500004 .7017150000

C 13.89145800001 .51152400003 .3990980000

C 12.52601500001 .40863000003 .0721530000

C 14.72098500002 .48596100002 .6154690000

H 14.67409400003 .46555300003 .1143740000

H 15.77676300002 .20700400002 .6280360000

C 14.21616600002 .634447700001 .2106820000

C 12.83882600002 .44787700000 .9910210000

C 15.03811300002 .99309100000 .1523310000

H 16.09865300003 .13465600000 .3346260000

C $14.52046800003 .1941600000-1.1224480000$

H $15.17050100003 .4885030000-1.9367030000$

C $13.16405500002 .9964110000-1.3454810000$ 
H $12.75269200003 .1172760000-2.3422930000$

C $12.32239400002 .5989360000-0.3139930000$

C $10.88723000002 .2714880000-0.5468170000$

H $10.66358600001 .2858570000-0.1301900000$

H $10.64957000002 .2479540000-1.6113790000$

C 9.54706000002 .36345700005 .4958210000

H 10.63846000002 .32145300005 .5851230000

C 9.11005100003 .74018600005 .9735700000

H 9.6002610000 4.54188100005 .4253660000

H 9.38186800003 .85439500007 .0245660000

H 8.03341100003 .88644000005 .9042570000

C 8.96184400001 .27072200006 .3816340000

H 7.8723160000 1.30074400006 .3977550000

H 9.3015890000 1.42116800007 .4079960000

H 9.26593000000 .26809000006 .0852600000

C 7.56169000001 .64178200003 .3383560000

H 7.4895520000 1.81015500002 .2648110000

C 7.17746500000 .19079700003 .5920060000

H $7.7558960000-0.50482500002 .9863340000$

H 6.12695700000 .05214300003 .3300080000

H 7.2907800000 -0.0955740000 4.6369040000

C 6.58795000002 .59858500004 .0106910000

H 6.52096500002 .42213900005 .0837930000

H 5.59014600002 .44355200003 .5966850000

H 6.84496900003 .64528300003 .8563580000

C $8.08770400002 .8946300000-0.3448890000$

H $8.11712100003 .1605660000-1.4050320000$

C $7.92094800001 .3795410000-0.2659760000$

H $8.47593800000 .8785180000-1.0574660000$

H $6.87014400001 .1097220000-0.3815180000$

H 8.26464400000 .95600200000 .6783200000

C 6.95201900003 .67646700000 .2970240000

H 6.92676300003 .56615400001 .3807750000

H $5.99181800003 .3326890000-0.0907340000$

H 7.0280940000 4.74243200000 .0845370000

C $10.13457900005 .0209890000-0.5724880000$

H $11.22469500005 .0576600000-0.4570260000$

C $9.81881700004 .9911230000-2.0614470000$

H $10.16979900004 .0886710000-2.5601020000$

H $10.29656800005 .8401010000-2.5530740000$

H $8.74641100005 .0791740000-2.2419010000$

C 9.56237100006 .27876700000 .0612850000

H 8.47219700006 .28830800000 .0499130000

H 9.8964230000 7.1523950000 -0.5011760000 
H 9.88359200006 .41570400001 .0915500000

C 9.12967300004 .84413100002 .8946360000

C 12.13143100005 .35146200003 .7646270000

O 12.16006100005 .34831900002 .2824800000

H 13.13393300005 .64003000004 .1220330000

H 11.84927400006 .20671000001 .9655200000

H 11.4136340000 6.1464630000 4.0487190000

\section{Ru-11}

O 1.092323000011 .16228000004 .6132280000

H 1.119027000010 .17411100004 .8385060000 H 0.98534300008 .73305800005 .0991790000 P - 2.31885200007 .81530300002 .8678740000 P 0.49324000006 .25352600004 .8590080000 O - 1.99916800008 .24283700006 .8709550000 N 0.80791700008 .26538100002 .4240750000 C - 1.68042100007 .97527700001 .1538150000 H - 1.08943100007 .06841000000 .9901440000 H - 2.51445500007 .96034800000 .4511690000 C -0.83839900009 .18596700000 .9346590000$ C - 1.281103000010 .22102700000 .1210400000 H - $2.272864000010 .1538920000-0.3150670000$ C $-0.465129000011 .3069980000-0.1674660000$ H -0.8190350000 $12.1050510000-0.8076990000$ C 0.824049000011 .33493200000 .3477660000 H 1.487162000012 .15885000000 .1028030000 C 1.287516000010 .33153800001 .1886100000 C 0.43927200009 .26314200001 .5373330000 C 2.668323000010 .38304600001 .7734120000 H 3.375483000010 .78981400001 .0452460000 H 2.6868270000 11.09895700002 .6062830000 C 3.13100200009 .04359000002 .2652670000 C 4.47931700008 .76984900002 .4495140000 H 5.20765900009 .52829400002 .1796300000 C 4.90366600007 .56356300002 .9910660000 H 5.95812600007 .36781600003 .1388430000 C 3.95606400006 .60933900003 .3359520000 H 4.26937100005 .65552100003 .7477550000 C 2.60022100006 .83835300003 .1355340000 C 2.16654800008 .07625100002 .6085600000 C 1.59193100005 .78816500003 .4593380000 H 2.08579500004 .84397700003 .6899640000 H 0.91730500005 .61726400002 .6141760000 
C -3.1283770000 6.15010400002 .8494370000

H -2.2431350000 5.50408000002 .8258200000

C -3.9631570000 5.77126800001 .6344910000

H -4.9224420000 6.28284800001 .6109960000

H -4.1728920000 4.70024600001 .6689390000

H -3.4511880000 5.96594100000 .6933540000

C -3.8817140000 5.89641400004 .1465290000

H -3.3131930000 6.2041030000 5.0259720000

H - 4.11319700004 .83510900004 .2558700000

H -4.8288100000 6.43780000004 .1591580000

C -3.6060400000 9.1519870000 2.9205450000

H -3.0409670000 9.99866100002 .5139080000

C -4.03119400009 .52044500004 .3333980000$

H - 4.49163800008 .68182700004 .8576100000

H -4.7692360000 10.32419400004 .3000780000

H -3.1933510000 9.8710380000 4.9326900000

C -4.81610600008 .92935300002 .0270590000$

H -4.5507720000 8.61807200001 .0169290000

H -5.3830760000 9.8584660000 1.9416650000

H -5.4901910000 8.18173600002 .4457260000

C 1.69772700006 .34058000006 .2711960000

H 2.4528060000 7.0102670000 5.8417220000

C 1.16775900007 .00865000007 .5301790000

H 0.80151400008 .01514100007 .3375390000

H 1.97354800007 .08978200008 .2619460000

H 0.36548600006 .43972900007 .9987230000

C 2.37872900005 .02080400006 .6034660000

H 1.70401900004 .33140600007 .1109860000

H 3.21357000005 .20679700007 .2817040000

H 2.7819970000 4.51493800005 .7272180000

C -0.59932700004 .77371200005 .1139120000$

H - 1.40498400004 .96263200004 .4042390000

C - 1.22061200004 .73657200006 .5025950000

H - 0.49399800004 .45073100007 .2632820000

H -2.0193250000 3.99301500006 .5256290000

H - 1.65602000005 .69076700006 .7966770000

C 0.01139500003 .42913000004 .7426290000

H 0.33485100003 .39259700003 .7037200000

H - 0.74199700002 .64978000004 .8727130000

H 0.86005800003 .16304500005 .3702060000

C -0.058926000011 .40228800004 .0519980000$

H - 0.186192000012 .45788200003 .7843970000

C - 1.38751700008 .24383100005 .8734710000

$\mathrm{Ru}-0.42840900008 .25556000004 .3364570000$ 
O -0.9325370000 10.57405400003 .8405390000

\section{Ru-12}

Ru 8.719215000012 .398146000013 .1372780000 P 7.731518000014 .188571000012 .1137510000 P 8.362189000010 .617326000011 .7127640000 O 9.748602000013 .569933000014 .7825430000 O 10.208988000011 .414716000014 .6737180000 O 6.212363000011 .819693000014 .6655400000 N 10.538915000012 .813562000011 .9614140000 C 8.942296000014 .850873000010 .8988380000 H 9.0412370000 14.076797000010 .1315100000 H 8.528693000015 .740700000010 .4246510000 C 10.266385000015 .155421000011 .5209000000 C 10.758533000016 .454696000011 .5713480000 H 10.151314000017 .261075000011 .1738690000 C 12.020829000016 .722095000012 .0832570000 H 12.398289000017 .736584000012 .1015980000 C 12.799773000015 .673843000012 .5604780000 H 13.788669000015 .871902000012 .9612830000 C 12.323976000014 .371427000012 .5567980000 C 11.043822000014 .098577000012 .0385630000 C 9.797572000010 .533449000010 .5709820000 H 9.71041800009 .63208000009 .9621490000 H 9.6892650000 11.39616700009 .9082060000 C 11.116963000010 .596367000011 .2640860000 C 12.01911500009 .540077000011 .2286300000 H 11.73089300008 .618888000010 .7327750000 C 13.28835400009 .669591000011 .7772650000 H 13.98861600008 .845149000011 .7323420000 C 13.659342000010 .874038000012 .3647770000 H 14.654955000010 .989824000012 .7808760000 C 12.766340000011 .931893000012 .4516920000 C 11.471760000011 .792380000011 .9207780000 C 13.096961000013 .223940000013 .1394970000 H 14.170866000013 .419984000013 .1140160000 H 12.849503000013 .133807000014 .2080320000 C 6.102084000014 .093576000011 .2389410000 H 6.119891000013 .089463000010 .8183200000 C 5.946730000015 .065888000010 .0786280000 H 4.971310000014 .91089400009 .6141380000 H 6.699844000014 .91346200009 .3070090000 H 5.992829000016 .107070000010 .3954710000 
C 4.917159000014 .158371000012 .1913550000

H 4.767491000015 .162130000012 .5874990000 H 5.0147270000 13.474523000013 .0337500000 H 4.008451000013 .884235000011 .6529580000 C 7.6767170000 15.566462000013 .3580850000 H 8.743833000015 .675906000013 .5829620000 C 6.971775000015 .248525000014 .6677850000 H 7.0964560000 16.0901270000 15.3515340000 H 7.3870240000 14.371357000015 .1587920000 H 5.901302000015 .094761000014 .5417250000 C 7.188167000016 .888369000012 .7791670000 H 6.114393000016 .877023000012 .5918400000 H 7.6891280000 17.1601660000 11.8512260000 H 7.3807130000 17.687822000013 .4969340000 C 8.60163400009 .053329000012 .6943010000 H 9.6656010000 9.1258580000 12.9484460000 C 7.82482600008 .965322000013 .9977460000 H 6.74669200009 .013241000013 .8431870000 H 8.10330900009 .748075000014 .7000810000 H 8.03819700008 .009446000014 .4792630000 C 8.40176800007 .792663000011 .8642390000 H 8.79024100006 .933214000012 .4127450000 H 8.91418700007 .821294000010 .9032620000 H 7.3440690000 7.6022920000 11.6769940000 C 6.919904000010 .321461000010 .6034620000 H 7.0352850000 9.2796800000 10.2929260000 C 5.6024410000 10.435599000011 .3541870000 H 5.459880000011 .416544000011 .8068460000 H 5.53081900009 .698201000012 .1535830000 H 4.766984000010 .263347000010 .6740250000 C 7.0029240000 11.17811700009 .3423070000 H 6.028712000011 .22597100008 .8537500000 H 7.707659000010 .75783300008 .6266130000 H 7.3226790000 12.2022950000 9.5355760000 C 10.355563000012 .545715000015 .1986420000 H 11.033916000012 .653341000016 .0624510000 C 7.1914900000 12.043080000014 .0763220000

\section{Ru-13}

Ru 8.723649000012 .381232000013 .1057190000 P 7.773711000014 .202449000012 .1727170000 P 8.339270000010 .608346000011 .6447040000 O 11.053671000013 .597931000016 .3286270000 
O 9.637835000013 .696555000014 .6108320000

O 6.190309000011 .809453000014 .5951550000

N 10.546838000012 .759667000011 .9497310000

C 9.005843000014 .875976000010 .9907870000

H 9.0884260000 14.128559000010 .1958400000

H 8.611972000015 .791921000010 .5507170000

C 10.332951000015 .124224000011 .6304750000

C 10.848034000016 .408488000011 .7621980000

H 10.257354000017 .249867000011 .4158810000

C 12.112436000016 .618954000012 .2952840000

H 12.507250000017 .623287000012 .3811030000

C 12.871016000015 .529168000012 .7078670000

H 13.860897000015 .684131000013 .1246810000

C 12.372275000014 .238254000012 .6196120000

C 11.089408000014 .024687000012 .0850690000

C 9.738634000010 .542355000010 .4567570000

H 9.62776800009 .65968500009 .8246650000

H 9.624512000011 .42502800009 .8213120000

C 11.071731000010 .563152000011 .1258030000

C 11.96247400009 .502576000011 .0090330000

H 11.65673700008 .619393000010 .4579530000

C 13.24073300009 .581724000011 .5447040000

H 13.93148400008 .755802000011 .4320760000

C 13.632071000010 .738951000012 .2080300000

H 14.634925000010 .817612000012 .6150410000

C 12.752895000011 .798245000012 .3781020000

C 11.450297000011 .712604000011 .8515150000

C 13.116734000013 .041245000013 .1359310000

H 14.195166000013 .210526000013 .1159060000

H 12.869511000012 .899582000014 .1981570000

C 6.177933000014 .053912000011 .2529370000

H 6.238428000013 .043864000010 .8501600000

C 6.045293000015 .008498000010 .0743190000

H 5.096598000014 .81699000009 .5701120000

H 6.8362130000 14.87370600009 .3384470000

H 6.043554000016 .053206000010 .3829910000

C 4.955341000014 .109199000012 .1572540000

H 4.773756000015 .114782000012 .5346720000

H 5.026858000013 .436995000013 .0107900000

H 4.074233000013 .815831000011 .5843730000

C 7.6510210000 15.573199000013 .4107040000

H 8.708454000015 .704306000013 .6633020000

C 6.913630000015 .235691000014 .6969790000

H 7.0136800000 16.070275000015 .3930630000 
H 7.3225230000 14.3558960000 15.1881970000

H 5.847987000015 .076657000014 .5385550000 C 7.142845000016 .879416000012 .8125360000 H 6.077303000016 .839203000012 .5870580000 H 7.6686790000 17.1687770000 11.9040710000 H 7.2873920000 17.681455000013 .5385060000 C 8.66430100009 .069364000012 .6451690000 H 9.73808400009 .169280000012 .8419210000 C 7.95938600009 .001924000013 .9903610000 H 6.87439100009 .046418000013 .8960670000 H 8.2726910000 9.8005040000 14.6618340000 H 8.20235700008 .056854000014 .4790690000 C 8.45224500007 .786296000011 .8545980000 H 8.90225200006 .949988000012 .3916510000 H 8.89988900007 .813311000010 .8614250000 H 7.3911390000 7.5606880000 11.7393150000 C 6.864223000010 .247609000010 .5969630000 H 6.98584700009 .193797000010 .3329640000 C 5.570082000010 .381624000011 .3835150000 H 5.408537000011 .393339000011 .7547650000 H 5.55143100009 .714266000012 .2453660000 H 4.719435000010 .124445000010 .7508050000 C 6.886022000011 .03717300009 .2918100000 H 5.913413000010 .97121700008 .8023110000 H 7.6270890000 10.63904100008 .6006670000 H 7.1124130000 12.09507900009 .4253290000 C 10.395188000013 .077431000015 .4250200000 H 10.447844000011 .970006000015 .2829770000 C 7.1801750000 12.038106000014 .0250790000

\footnotetext{
Ru-15

O -0.735437000010 .44589000004 .0739290000$

H - 0.141376000010 .68118200003 .3428000000 H 0.99803200008 .50445100005 .2654490000 P -2.2972550000 7.7965130000 2.9493440000 P 0.58252900006 .08086700004 .8569120000 O -2.0422070000 7.9394210000 6.9238770000 N 0.89063600008 .21181800002 .5919840000 C - 1.57859500007 .71466300001 .2518390000 H -0.97585600006 .80111700001 .2358750000$ H - 2.36831500007 .60880800000 .5072740000 C -0.73759300008 .91067800000 .9452910000$ C - $1.14355400009 .8224630000-0.0230020000$
} 
H -2.0819560000 $9.6469630000-0.5385360000$

C $-0.364298000010 .9205350000-0.3601190000$

H -0.6929320000 $11.6097710000-1.1274710000$

C 0.843043000011 .11847600000 .2959630000

H 1.463550000011 .97387300000 .0488970000

C 1.265962000010 .24803100001 .2899920000

C 0.48251200009 .12364100001 .6318750000

C 2.514852000010 .49441400002 .0846750000

H 3.228803000011 .09701500001 .5197590000

H 2.2592890000 11.10353700002 .9670270000

C 3.12876600009 .21060500002 .5524630000

C 4.48867300009 .07397100002 .7948200000

H 5.1478400000 9.90794600002 .5756790000

C 5.00426000007 .90456400003 .3393550000

H 6.06448300007 .81307000003 .5386130000

C 4.14348700006 .85054600003 .6203610000

H 4.53253200005 .92399500004 .0305680000

C 2.78409200006 .94480900003 .3523080000

C 2.25566100008 .14520300002 .8297380000

C 1.86069900005 .79736800003 .5635070000

H 2.40729800004 .88491400003 .8090130000

H 1.29457900005 .61211000002 .6476840000

C -3.4703700000 6.35890600003 .0700830000

H - 2.82766400005 .54941100003 .4142760000

C -4.10025200005 .89813500001 .7652540000$

H -4.7250320000 6.66145100001 .3039820000

H -4.7369560000 5.03190800001 .9573890000

H -3.3521790000 5.59029700001 .0352880000

C -4.50908000006 .58497700004 .1594610000$

H -4.0670680000 6.95861700005 .0846730000

H -5.0097250000 5.64362200004 .3933520000

H -5.2785100000 7.2911810000 3.8479690000

C -3.31917700009 .34683100002 .8364980000$

H -2.5642680000 10.05388700002 .4782080000

C -3.8123820000 9.86728800004 .1797240000

H -4.67212700009 .30804800004 .5465740000$

H -4.1283440000 10.90780700004 .0778910000

H -3.0409340000 9.83574500004 .9474120000

C -4.44341900009 .32029900001 .8123820000$

H - 4.11770900008 .96667700000 .8345100000

H -4.8400210000 10.32891200001 .6759790000

H -5.2737610000 8.69262500002 .1366410000

C 1.60608500005 .99638500006 .4080020000

H 2.44311000006 .65093300006 .1350150000 
C 0.95129000006 .58157800007 .6476750000

H 0.68501500007 .62842000007 .5158680000

H 1.64463600006 .52329900008 .4886520000

H 0.05179300006 .03586200007 .9350360000

C 2.1628770000 4.61090500006 .7004190000

H 1.39351300003 .94364200007 .0916080000

H 2.94053200004 .68283200007 .4624750000

H 2.6088090000 4.13430400005 .8275090000

C -0.36954000004 .49347700004 .8102650000$

H 0.31242300003 .73849500005 .2085450000

C -0.70874900004 .09364400003 .3775470000$

H - 1.00105000004 .94386800002 .7581680000

H - 1.53426000003 .37987100003 .3649700000

H 0.13904600003 .61983100002 .8850560000

C - 1.58185900004 .56281100005 .7254530000

H - 1.29568800004 .72124300006 .7655930000

H -2.1485960000 3.63121100005 .6789050000

H -2.2556830000 5.37429600005 .4490450000

C - 0.413993000011 .25448500005 .2018390000

H - 0.940320000010 .80845100006 .0446590000

H 0.664708000011 .23125100005 .3826680000

C - 1.38312700007 .98967700005 .9569120000

$\mathrm{Ru}-0.37676800008 .08070100004 .4525170000$

O - 0.885524000012 .55595500005 .0489300000

H - 0.251634000013 .05447700004 .5191120000

$\mathbf{T S}_{2,3}$

S - 0.524340000011 .14305300003 .4259260000

P -2.1632690000 7.8929840000 2.8808080000

P 0.77959800006 .74842400004 .9379210000

O -1.74229200009.0653720000 6.7064340000

N 0.89017100008 .37965000002 .2350400000

C - 1.59940900007 .71757500001 .1417460000

H - 0.97827100006 .81577000001 .1445800000

$\mathrm{H}-2.46024600007 .52321200000 .5017380000$

C -0.81808000008 .87443000000 .6195290000$

C - $1.29561000009 .6522840000-0.4285620000$

H - $2.28324400009 .4432180000-0.8271890000$

C - $0.513542000010 .6513570000-0.9934130000$

H - $0.892284000011 .2413840000-1.8184520000$

C $0.767534000010 .8705910000-0.5016110000$

H $1.393936000011 .6398540000-0.9418740000$

C 1.255701000010 .13919800000 .5714700000 
C 0.45676300009 .14301900001 .1615270000

C 2.592488000010 .41239500001 .1917630000

H 3.284861000010 .84521400000 .4667420000

H 2.467444000011 .18078600001 .9696180000

C 3.16658300009 .17941300001 .8227720000

C 4.53246900008 .97136600001 .9430390000

H 5.21423000009 .69953900001 .5152550000

C 5.03201700007 .86700900002 .6231440000

H 6.10009700007 .72017100002 .7216640000

C 4.14480100006 .95098400003 .1710060000

H 4.51954400006 .07612200003 .6921150000

C 2.77027600007 .11173200003 .0361410000

C 2.26288500008 .24700000002 .3678690000

C 1.82260200006 .09264700003 .5734360000

H 2.3637160000 5.2122330000 3.9206340000

H 1.11537900005 .76962100002 .8025280000

C - 2.90647800006 .23435800003 .2312420000

H - 1.99978800005 .61947500003 .2657470000

C -3.8035620000 5.61471200002 .1693550000

H -4.77913100006 .09250600002 .1187080000$

H -3.9745820000 4.56572900002 .4192470000

H -3.3616570000 5.63791600001 .1746130000

C -3.5670870000 6.20467300004 .6011160000

H -2.9618030000 6.6904770000 5.3684200000

H -3.7431800000 5.1754980000 4.9196810000

H -4.5356800000 6.70576000004 .5765070000

C -3.4929030000 9.1748190000 2.7266140000

H -2.9475210000 9.96506000002 .1955980000

C -3.9372690000 9.7482040000 4.0616320000

H - 4.39630300008 .99520500004 .7032380000

H -4.6799590000 10.53179500003 .8999940000

H -3.1073780000 10.19311300004 .6049270000

C -4.6934530000 8.77898200001 .8809370000

H -4.4225410000 8.29220500000 .9446830000

H -5.2689720000 9.6714190000 1.6276700000

H -5.3621040000 8.11313800002 .4266260000

C 2.05518500007 .15582500006 .2262680000

H 2.73964100007 .77353600005 .6315100000

C 1.55466100008 .01938500007 .3735080000

H 1.10924100008 .94879500007 .0221640000

H 2.39400600008 .28517800008 .0185070000

H 0.82196700007 .50634900007 .9954590000

C 2.83792000005 .95882500006 .7456370000

H 2.2395040000 5.3454270000 7.4193410000 
H 3.70052600006 .30977100007 .3150590000

H 3.21517400005 .31738000005 .9501720000

C - 0.21427700005 .29700800005 .5281890000

H - 1.06744900005 .31258500004 .8498960000

C -0.75172100005 .50325700006 .9366520000$

H 0.0318520000 5.3949000000 7.6868240000

H - 1.51175100004 .75045300007 .1528670000

H - 1.21371700006 .48081600007 .0726030000

C 0.44974500003 .93564800005 .3764710000

H 0.71119500003 .71499500004 .3428790000

H - 0.24734400003 .16203400005 .7042090000

H 1.34927800003 .83541200005 .9812210000

C - 0.936595000012 .05357000004 .9606180000

H - 1.203921000013 .05398700004 .6172750000

H - 1.838062000011 .62483100005 .3995450000

C 0.173807000012 .12180900005 .9789950000

H 1.079903000012 .55596100005 .5558410000

H 0.427551000011 .13483200006 .3677240000

H - 0.128870000012 .73847400006 .8268250000

C - 1.17162500008 .89349500005 .7037010000

$\mathrm{Ru}-0.26075500008 .66290300004 .1441660000$

H 1.16936800009 .39306600004 .7128690000

H 0.633621000010 .23767200004 .1527870000

TS $_{\text {III }}$

S -0.6102170000 10.77887000004 .0432200000

H 0.720713000010 .98162400003 .9228950000

O 2.3879770000 10.79671000005 .1071350000

P -2.3583500000 7.8922580000 3.0524020000

P 0.55599200006 .22618700004 .7924710000

O - 1.96728400008 .27553600006 .9236120000

N 0.76052700008 .30270400002 .4534930000

C - 1.75151900007 .69224700001 .3266880000

H - 1.12878400006 .79254800001 .3391950000

H - 2.59086300007 .51164500000 .6547390000

C -0.95961500008 .87250500000 .8677270000$

C - $1.43823900009 .6998140000-0.1417170000$

H - $2.41175800009 .4883160000-0.5713670000$

C $-0.679240000010 .7554100000-0.6276410000$

H - $1.060220000011 .3821230000-1.4239330000$

C $0.581061000010 .9865190000-0.0910970000$

H $1.189260000011 .8034600000-0.4660840000$

C 1.076944000010 .20060900000 .9389290000 
C 0.30405600009 .13528400001 .4446380000

C 2.402348000010 .48239600001 .5828490000

H 3.087826000010 .94921800000 .8717630000

H 2.2707170000 11.23398600002 .3762170000

C 3.00607000009 .24509300002 .1776360000

C 4.37361500009 .11063300002 .3750010000

H 5.03053000009 .91367000002 .0560630000

C 4.90398900007 .98271000002 .9888600000

H 5.97103300007 .89760100003 .1510830000

C 4.05126900006 .95395900003 .3695820000

H 4.45306100006 .04829900003 .8127560000

C 2.68180300007 .04623200003 .1569160000

C 2.13672900008 .22394000002 .5988160000

C 1.76780600005 .90812800003 .4489620000

H 2.3271220000 5.0023400000 3.6895010000

H 1.15607000005 .69857100002 .5676610000

C -3.46342200006 .43883700003 .3866940000$

H -2.7687510000 5.70493900003 .7892610000

C -4.1136620000 5.8130590000 2.1629940000

H -4.7869370000 6.49623200001 .6469380000

H - 4.70398500004 .94797900002 .4723040000

H -3.3775160000 5.45861300001 .4423560000

C -4.47361500006 .73360400004 .4857220000$

H -4.0217090000 7.2268130000 5.3471120000

H -4.91280100005 .79861300004 .8382170000$

H -5.2916660000 7.3599540000 4.1310040000

C -3.4442190000 9.3920510000 2.8744040000

H - 2.735340000010 .09544000002 .4238730000

C -3.9128090000 9.9969040000 4.1902090000

H -4.7875580000 9.4820730000 4.5847680000

H -4.195018000011 .04067700004 .0404680000$

H -3.1484390000 9.97236800004 .9659360000

C -4.60646900009 .23783600001 .9047160000$

H - 4.30357700008 .83892200000 .9376200000

H -5.0604890000 10.21435500001 .7235900000

H -5.3871830000 8.59262400002 .3071910000

C 1.65522100006 .23548500006 .2924790000

H 2.46271100006 .89706000005 .9561870000

C 1.03953800006 .84935400007 .5392340000

H 0.71233200007 .87441800007 .3784700000

H 1.77899300006 .86463800008 .3419940000

H 0.18524900006 .27522100007 .9000240000

C 2.26590200004 .87865700006 .6112690000

H 1.53452000004 .20637800007 .0620470000 
H 3.07399500005 .00293400007 .3338550000

H 2.6865520000 4.3811230000 5.7376770000

C -0.35683100004 .61976500004 .8715130000$

H 0.38374200003 .89616600005 .2207170000

C - 0.80425800004 .17208100003 .4809730000

H - 1.11759400004 .99955800002 .8428190000

H - 1.64334100003 .47786600003 .5513900000

H 0.00070500003 .65973900002 .9561520000

C - 1.47006600004 .67388600005 .9077330000

H - 1.06878900004 .78441400006 .9152550000

H -2.0543560000 3.75237800005 .8878660000

H - 2.15751100005 .50419300005 .7443440000

C -0.7130120000 11.47153800005 .7308200000

H - 1.731981000011 .26651000006 .0587070000

H - 0.042280000010 .89079700006 .3631630000

C -0.401153000012 .94454000005 .7638300000$

H - 1.085972000013 .51571100005 .1379320000

H 0.615112000013 .14406700005 .4246240000

H - 0.490260000013 .31794400006 .7843420000

C 2.36944500009 .65752900005 .6001280000

H 1.08357600008 .78296600005 .1205050000

H 3.01601500008 .85827900005 .1979340000

C - 1.35419500008 .27512400005 .9295670000

$\mathrm{Ru}-0.41092600008 .29058800004 .3756250000$

H 2.0631940000 9.50363700006.6508200000

$\mathbf{T S}_{\mathrm{IV}}$

S -0.6486560000 10.80994500003 .9828500000

H 0.705782000010 .97100200004 .0075520000

O 2.266660000010 .78795500004 .9610900000

P -2.3506110000 7.89648200003 .0456550000

P 0.50911300006 .24461300004 .7796280000

O -2.0282060000 8.36090400006 .8838990000

N 0.74686900008 .33280100002 .4524240000

C - 1.74211900007 .67881300001 .3241430000

H - 1.11023800006 .78595600001 .3454000000

H -2.5833700000 7.47925300000 .6602150000

C - 0.96240700008 .85833200000 .8435280000

C -1.4399690000 9.6606580000 -0.1863770000

H -2.4109690000 $9.4372120000-0.6155570000$

C $-0.679824000010 .7057210000-0.6928900000$

H - $1.058590000011 .3138410000-1.5045010000$

C $0.579616000010 .9497550000-0.1590420000$ 
H $1.188428000011 .7574630000-0.5524650000$

C 1.072638000010 .18936100000 .8910840000

C 0.29637700009 .13901500001 .4197040000

C 2.395479000010 .48322500001 .5351510000

H 3.084084000010 .93790200000 .8194550000

H 2.256232000011 .24511100002 .3164980000

C 2.99622500009 .25631400002 .1541990000

C 4.36296800009 .11729500002 .3510480000

H 5.02450100009 .90861300002 .0131790000

C 4.88749800007 .99917800002 .9883040000

H 5.95426000007 .91176100003 .1510610000

C 4.03083700006 .98201900003 .3892380000

H 4.42895900006 .08086800003 .8444920000

C 2.66128500007 .07745100003 .1750790000

C 2.12261400008 .24948100002 .6004780000

C 1.74437300005 .93861900003 .4573690000

H 2.3014740000 5.03220100003 .6987600000

H 1.14614500005 .73252600002 .5657570000

C -3.4288730000 6.43297800003 .4121950000

H - 2.72246100005 .72225300003 .8334730000

C -4.0611310000 5.76889400002 .1989420000

H - 4.75187300006 .42511100001 .6711900000

H -4.6290350000 4.89511700002 .5251780000

H -3.3162940000 5.42000200001 .4847170000

C - 4.45095600006 .73020900004 .4993570000

H -4.0154590000 7.2508810000 5.3525680000

H - 4.87080400005 .79291600004 .8688290000

H -5.2805920000 7.3302400000 4.1268120000

C - 3.45827300009 .37593600002 .8430240000

H - 2.759271000010 .08454800002 .3858640000

C -3.9508560000 9.99032900004 .1453560000

H -4.8318630000 9.4765860000 4.5270230000

H - 4.231950000011 .03225500003 .9819110000

H -3.2010290000 9.97344500004 .9342570000

C - 4.61078000009 .18350100001 .8678390000

H - 4.29492700008 .78141000000 .9064600000

H -5.0835160000 10.14859400001 .6744360000

H -5.3801240000 8.52723200002 .2740430000

C 1.56477700006 .24449600006 .3088040000

H 2.34608700006 .96489400006 .0381290000

C 0.86705500006 .75654200007 .5581670000

H 0.5047510000 7.7738780000 7.4416900000

H 1.57054200006 .75935100008 .3927830000

H 0.02649900006 .12510500007 .8466100000 
C 2.23800300004 .90777700006 .5857010000

H 1.53048600004 .17814000006 .9820130000 H 3.01393000005 .04247800007 .3408630000 H 2.7140040000 4.47316300005 .7074220000 C - 0.39526600004 .63071300004 .8271680000 H 0.35387800003 .91420300005 .1728410000 C - 0.81233100004 .18885900003 .4239190000 H - 1.12167300005 .01348000002 .7812680000 H - 1.64503400003 .48549700003 .4751090000 H 0.00839100003 .68724200002 .9134080000 C - 1.51899500004 .64763000005 .8576510000 H - 1.12381000004 .58932000006 .8712570000 H -2.1784870000 3.78955000005 .7172740000 H - 2.13356300005 .54712100005 .8076570000 C -0.874929000011 .55543600005 .6383810000$ H - 1.920305000011 .39073300005 .8962120000 H -0.2689820000 10.98347900006 .3402960000 C - 0.522835000013 .02015700005 .6473620000 H - 1.137987000013 .58671300004 .9490700000 H 0.522712000013 .18048500005 .3847240000 H -0.6824350000 13.43240200006 .6441200000 C 2.25564800009 .65482400005 .5121710000 H 1.11209800008 .94351100005 .1434230000 H 2.9448340000 8.86357900005 .1739620000 C - 1.39862000008 .34106100005 .9019910000 $\mathrm{Ru}-0.43563100008 .33717800004 .3562920000$ O 2.20577200009 .60228400006 .9042700000 H 1.856005000010 .45691100007 .1986070000

$\mathbf{T S}_{5,6}$ O -0.2062540000 10.77414900003 .8223680000 P -2.0613490000 7.8741260000 3.0433720000 P 0.80639500006 .54090900004 .9414160000 O -1.6531950000 8.71780200006 .8916110000 N 0.96661800008 .35807000002 .4071240000 C - 1.51430700007 .69566400001 .2976200000 H - 0.89994700006 .78892700001 .2994150000 H - 2.38114500007 .50503800000 .6645210000 C -0.72178300008 .84895800000 .7717440000$ C - $1.14882300009 .5865720000-0.3272620000$ H - $2.10158100009 .3434130000-0.7851090000$ C $-0.356395000010 .5858510000-0.8757010000$ H - $0.699964000011 .1412910000-1.7391400000$ 
C $0.887923000010 .8531120000-0.3166640000$

H $1.517785000011 .6299570000-0.7379730000$

C 1.335538000010 .14892300000 .7899930000

C 0.53221000009 .13690900001 .3487990000

C 2.640830000010 .43877600001 .4697660000

H 3.337501000010 .94057500000 .7959640000

H 2.4606860000 11.14849900002 .2911740000

C 3.23623500009 .18582600002 .0391260000

C 4.60238300008 .97379000002 .1468240000

H 5.28447000009 .72557100001 .7629780000

C 5.10139900007 .82946400002 .7592210000

H 6.16970700007 .67937600002 .8503460000

C 4.21710000006 .87533800003 .2446270000

H 4.59474200005 .96788100003 .7041650000

C 2.84268300007 .04254100003 .1179260000

C 2.33841800008 .22030500002 .5299980000

C 1.88399400005 .98832800003 .5593340000

H 2.41570900005 .08426000003 .8559800000

H 1.19807600005 .72382700002 .7481490000

C -3.0081480000 6.31755800003 .3549240000

H -2.2396480000 5.56446900003 .1400640000

C -4.18237400006 .06118900002 .4220030000$

H -5.0172350000 6.72468800002 .6460560000

H -4.5397120000 5.03960000002 .5633350000

H -3.9295650000 6.17337500001 .3689230000

C -3.43840200006 .16631100004 .8078230000$

H -2.6741930000 6.49220600005 .5109570000

H -3.6644780000 5.1209570000 5.0248930000

H - 4.34107700006 .73944600005 .0144820000

C -3.2160440000 9.3271320000 3.0011440000

H - 2.504434000010 .16204100002 .9792760000

C -4.05843400009 .45020200004 .2623330000$

H - 4.87159400008 .72342500004 .2632160000

H - 4.515199000010 .44026000004 .3050000000

H -3.4888450000 9.31382700005 .1800200000

C -4.0981750000 9.4437420000 1.7661640000

H -3.5318540000 9.44154800000 .8385200000

H -4.6424790000 10.38930400001 .8073370000

H -4.8406410000 8.64953200001 .7119340000

C 2.06814700006 .86502900006 .2687460000

H 2.75541100007 .52411500005 .7234600000

C 1.56435800007 .64244000007 .4744990000

H 1.15126200008 .61029800007 .1958210000

H 2.3977390000 7.8310730000 8.1535050000 
H 0.80720200007 .09915100008 .0387800000

C 2.85144100005 .63321400006 .7017920000

H 2.2496470000 4.96655500007 .3194140000

H 3.70625300005 .94368500007 .3053290000

H 3.24052800005 .05700900005 .8636350000

C - 0.19121300005 .04872600005 .4007990000

H - 1.04298100005 .12334700004 .7221010000

C - 0.71630900005 .13210100006 .8270860000

H 0.07325100004 .94038600007 .5538290000

H - 1.48839300004 .37659000006 .9808390000

H - 1.15564200006 .10110500007 .0629330000

C 0.47464100003 .70618200005 .1323430000

H 0.72069100003 .56826800004 .0810070000

H -0.2155220000 2.90707100005 .4091680000

H 1.38342500003 .56309800005 .7144800000

C -0.854687000011 .56698800004 .7824490000$

H - 1.930421000011 .35735700004 .8396290000

H - 0.444328000011 .42325000005 .7918470000

C - 1.08934800008 .63313500005 .8750620000

$\mathrm{Ru}-0.17533400008 .51766900004 .2985200000$

H 1.27997300009 .42808600004 .9101970000

H 0.787265000010 .12064300004 .4684940000

H - 0.738337000012 .62828000004 .5323610000

$\mathbf{T S}_{6,7}$

Ru -0.3481240000 -0.2738470000 0.6965800000

P - $1.41873800001 .6575960000-0.3193380000$

P - $0.6602880000-2.0938270000-0.6936440000$

O 0.66253800000 .98448900002 .2038640000

O -2.7869450000 -0.9397920000 2.2872280000

N $1.45023300000 .2222460000-0.5516680000$

C $-0.24125500002 .2553290000-1.5993240000$

H - $0.17750500001 .4485830000-2.3361830000$

H - $0.64981300003 .1331380000-2.1003570000$

C $1.10562800002 .5640280000-1.0328700000$

C $1.57577200003 .8734090000-1.0300070000$

H $0.93617900004 .6551600000-1.4260350000$

C $2.84701500004 .1832340000-0.5707340000$

H $3.20379600005 .2051250000-0.5917040000$

C $3.65832300003 .1612880000-0.0942890000$

H 4.65578000003 .38453400000 .2707130000

C $3.20798900001 .8508260000-0.0503270000$

C $1.91845500001 .5276100000-0.5210790000$ 
C $0.7712620000-2.1498760000-1.8379440000$

H $0.7147070000-3.0705050000-2.4216630000$

H $0.6182070000-1.3097470000-2.5201750000$

C $2.0951730000-2.0084080000-1.1700960000$

C $3.0277580000-3.0377400000-1.1759690000$

H $2.7560390000-3.9876810000-1.6252220000$

C $4.3032430000-2.8490240000-0.6595060000$

H $5.0270340000-3.6538330000-0.6811850000$

C $4.6451520000-1.6090380000-0.1348030000$

H $5.6432710000-1.44294800000 .2578110000$

C $3.7218740000-0.5738060000-0.0787210000$

C $2.4210420000-0.7684190000-0.5768610000$

C 4.03766300000 .75260200000 .5449220000

H 5.10283400000 .97993000000 .4637460000

H 3.83854500000 .70293700001 .6254880000

C - $3.07665700001 .5685270000-1.1477700000$

H -3.0796050000 $0.5668800000-1.5764380000$

C -3.2832190000 $2.5451900000-2.2952010000$

H -4.2779930000 $2.3947950000-2.7190270000$

H -2.5644270000 $2.3928750000-3.0993820000$

H -3.2201400000 $3.5856990000-1.9793150000$

C - $4.21687000001 .6235470000-0.1417970000$

$\mathrm{H}-4.36878600002 .63167100000 .2429440000$

H -4.0584430000 0.95952200000 .7083720000

H -5.1458780000 $1.3187150000-0.6264970000$

C - 1.42515300003 .06562000000 .8887900000

H - 0.35149000003 .15509600001 .0906140000

C - 2.10805600002 .75954800002 .2137700000

H - 1.86104600003 .53914600002 .9367350000

H - 1.78526800001 .80988700002 .6373050000

H -3.1930250000 2.73981100002 .1245050000

C - 1.90337400004 .39161200000 .3163050000

H - 2.98204600004 .39639100000 .1576210000

H - $1.42251100004 .6446120000-0.6280010000$

H - 1.67911200005 .19596900001 .0197760000

C $-0.5034590000-3.72427700000 .1832350000$

H $0.5707930000-3.72994400000 .4083270000$

C - $1.2471180000-3.82452000001 .5042390000$

H - $2.3262370000-3.73092100001 .3792470000$

H -0.9210630000 -3.0695460000 2.2177790000

H - $1.0580800000-4.80028700001 .9546210000$

C $-0.8079200000-4.9221040000-0.7031140000$

H - $0.4727800000-5.8353990000-0.2094630000$

H - $0.3087460000-4.8757640000-1.6708820000$ 
H - $1.8791060000-5.0286390000-0.8799690000$

C $-2.1053730000-2.2647230000-1.8300780000$

H - $2.0319770000-3.2795650000-2.2289870000$

C $-3.4144010000-2.1538440000-1.0642290000$

H -3.5064120000 - $1.2011590000-0.5419000000$

H -3.5136980000 -2.9427900000 -0.3184020000

H -4.2610520000 -2.2388270000 - 1.7472650000

C - $1.9946290000-1.2985850000-3.0046580000$

H - $2.9676420000-1.1610950000-3.4789960000$

H - $1.3103730000-1.6732600000-3.7642040000$

H - $1.6321620000-0.3127690000-2.7090120000$

C $1.0059580000-0.24051200002 .4360700000$

H $0.5807080000-1.49860700001 .2938970000$

C - $1.8367410000-0.68428200001 .6638500000$

H $2.0428480000-0.54377500002 .2372520000$

H $0.5515330000-0.76804700003 .2860910000$

$\mathbf{T S}_{\mathbf{8}, 9}$

O 0.85570300009 .56858400005 .5388920000

P -2.3026980000 7.78749200002 .8949720000

P 0.34848800006 .04881500004 .8370180000

O - 2.52008800007 .78586300006 .7250840000

N 0.74736000008 .34429200002 .6940080000

C - 1.57724000008 .01221000001 .2229140000

H -0.9391000000 7.13797600001 .0706630000

H -2.3832660000 7.97012500000 .4881070000

C -0.76626000009 .25304000001 .0721080000$

C - 1.128452000010 .26666800000 .1939380000

H -2.0593920000 $10.1771730000-0.3564270000$

C $-0.297398000011 .3598360000-0.0148730000$

H - $0.582487000012 .1375840000-0.7119220000$

C 0.910183000011 .43802200000 .6685690000

H 1.571427000012 .28324400000 .5068680000

C 1.279557000010 .46127400001 .5825520000

C 0.43351700009 .36163200001 .8061640000

C 2.529252000010 .55225600002 .4064330000

H 3.299085000011 .13348800001 .8949780000

H 2.3080780000 11.11525200003 .3258190000

C 3.04081200009 .19062300002 .7796930000

C 4.38663900008 .93775700003 .0015490000

H 5.10232100009 .74120800002 .8607220000

C 4.82010400007 .68597100003 .4201480000

H 5.87156900007 .50215800003 .6006240000 
C 3.89166400006 .66927100003 .5956700000

H 4.21967400005 .68164300003 .9021160000

C 2.53952600006 .87815800003 .3495690000

C 2.09518500008 .15898700002 .9528690000

C 1.55577500005 .76183100003 .4770920000

H 2.0732010000 4.81593800003 .6365050000

H 0.95341900005 .66735000002 .5682530000

C -3.2742210000 6.23018900002 .7110930000

H -4.0878960000 6.49234000002 .0299530000

C -3.8915830000 5.80895500004 .0354370000

H -3.1421890000 5.65738300004 .8123120000

H -4.4385720000 4.87237200003 .9166350000

H -4.5950300000 6.5549740000 4.4059360000

C -2.4444030000 5.14312300002 .0328500000

H - 2.41499700005 .28544700000 .9537490000

H - 2.87798400004 .16001900002 .2221280000

H - 1.40884900005 .11693400002 .3758800000

C - 3.54511000009 .17302400002 .9365740000

H - 2.934214000010 .00748400002 .5702530000

C -4.06031100009 .55227900004 .3146890000$

H -4.59212500008 .73201100004 .7976260000$

H - 4.763664000010 .38148800004 .2220820000

H -3.2622910000 9.8780810000 4.9787170000

C - 4.70239000008 .97107100001 .9690190000

H -4.3827950000 8.66620700000 .9728310000

H -5.2526210000 9.90691700001 .8606810000

H -5.4073340000 8.22580000002 .3401680000

C 1.47977300006 .25716100006 .2947670000

H 2.13804400007 .05361900005 .9253430000

C 0.81630200006 .78248400007 .5589470000

H 0.27903800007 .71273100007 .3866810000

H 1.58416500006 .98693100008 .3073760000

H 0.12432200006 .06612500007 .9985960000

C 2.3424020000 5.03851900006 .5913550000

H 1.75878900004 .23203100007 .0358120000

H 3.11879900005 .30750300007 .3101250000

H 2.84222400004 .64412500005 .7075690000

C - 0.60269600004 .46848400005 .0286730000

H - 1.42678600004 .60202600004 .3290000000

C - 1.21556200004 .32510200006 .4139050000

H - 0.46758000004 .07423100007 .1655440000

H - 1.94739600003 .51558800006 .4032390000

H - 1.73280800005 .22716800006 .7408030000

C 0.14231100003 .21105100004 .6067070000 
H 0.43570100003 .23853400003 .5581950000

H - 0.51371800002 .34813700004 .7355410000

H 1.03578600003 .03037400005 .2030860000

C - 1.78822100007 .92957100005 .8308480000

$\mathrm{Ru}-0.63875300008 .13986100004 .4264790000$

H -0.1985380000 9.9374620000 4.7229830000

H 1.63490600009 .53231700004 .9676660000

H -0.9705970000 9.90419400004 .1760850000

$\mathbf{T S}_{9,10}$

O 0.68773100008 .79039700005 .7015470000

P - 2.17798700007 .19554200002 .7313620000

P 0.40586500005 .69938100004 .8413900000

O -2.6825870000 6.9851170000 6.5695830000

N 0.79066200008 .08509500002 .8614430000

C - 1.34277700007 .61785400001 .1499270000

H - 0.59727000006 .83204700001 .0003800000

H - 2.06686900007 .54868300000 .3365310000

C -0.66921100008 .94829900001 .1681530000$

C - 1.06367800009 .98648200000 .3329100000

H - $1.92698200009 .8455630000-0.3093100000$

C -0.341379000011 .17158700000 .2798520000$

H - $0.648903000011 .9683810000-0.3855100000$

C 0.792507000011 .31787200001 .0718820000

H 1.372185000012 .23411200001 .0245880000

C 1.185292000010 .31692200001 .9476530000

C 0.43983200009 .12652400002 .0209070000

C 2.350485000010 .45579800002 .8829730000

H 3.085708000011 .16529900002 .4984580000

H 1.991292000010 .88862500003 .8292730000

C 2.97714500009 .12383800003 .1784320000

C 4.32044900008 .97019600003 .4853130000

H 4.97183600009 .83766700003 .4527560000

C 4.83145800007 .73223300003 .8613610000

H 5.8801950000 7.6256410000 4.1081470000

C 3.98571000006 .63215300003 .9149390000

H 4.37590100005 .65927500004 .1947020000

C 2.64173100006 .74704500003 .5795930000

C 2.12286100008 .00626900003 .2144980000

C 1.73141600005 .56309400003 .5753660000

H 2.29018400004 .64180300003 .7384170000

H 1.20863800005 .47449100002 .6179750000

C -3.0257680000 5.60926400002 .3196190000 
H -3.7417150000 5.88366800001 .5406130000

C -3.82125300005 .07199300003 .5009520000$

H -3.2215010000 4.95607600004 .4036470000

H - 4.24322800004 .09550700003 .2584360000

H - 4.65105300005 .73246800003 .7515310000

C -2.0455030000 4.61258900001 .6986290000

H - 1.93055500004 .79205400000 .6307930000

H -2.4080090000 3.59100400001 .8227880000

H - 1.04580600004 .65847000002 .1322390000

C -3.51645500008 .49358800002 .7726220000$

H -2.9404070000 9.40183200002 .5606200000

C -4.1998930000 8.68851300004 .1172150000

H -4.6890710000 7.7812020000 4.4722300000

H -4.9719220000 9.45419600004 .0215500000

H -3.5086830000 9.02441400004 .8874050000

C -4.54821800008 .32341800001 .6659770000$

H -4.10462400008 .15604100000 .6849870000$

H -5.1566140000 9.2265450000 1.5960670000

H -5.2270640000 7.4953960000 1.8753590000

C 1.39106800005 .79350600006 .4102890000

H 2.02989700006 .65714500006 .1928390000

C 0.60435100006 .11099300007 .6740650000

H - 0.10357500006 .92906200007 .5492920000

H 1.29811100006 .39930500008 .4653890000

H 0.04565700005 .25065000008 .0380190000

C 2.29344200004 .58641800006 .6340680000

H 1.72046300003 .69495500006 .8891570000

H 2.96130700004 .78973000007 .4731400000

H 2.91847000004 .35236000005 .7741200000

C - 0.52449100004 .09864200004 .8096010000

H - 1.32074300004 .30816700004 .0984630000

C - 1.18806800003 .75945400006 .1362050000

H - 0.46455200003 .42337800006 .8780750000

H - 1.89525900002 .94260300005 .9828700000

H - 1.74344300004 .59497100006 .5594230000

C 0.27063000002 .92018300004 .2661270000

H 0.62376700003 .08955700003 .2501290000

H - 0.37351600002 .03922700004 .2430650000

H 1.13186500002 .67637200004 .8875560000

C -1.8961320000 7.2496390000 5.7497940000

$\mathrm{Ru}-0.66977400007 .65134600004 .4615430000$

H 0.63885500008 .49797200006 .6189360000

O - 1.210938000010 .27701900004 .5657480000

C -0.710183000010 .34650200005 .6922640000$ 
H 0.039072000011 .11607600005 .9381870000

H - 1.19451100009 .88824700006 .5710590000

$\mathrm{TS}_{10,11}$

Ru 10.68265100003 .68251200002 .3871710000

O 12.04690100004 .26928800004 .0764870000

P 9.42726700002 .11765100003 .6804400000

P 10.08373500003 .45459200000 .1899780000

O 8.69294800005 .87730400002 .7566660000

N 12.16301900002 .01619200002 .0847590000

C 10.39153300000 .55517500003 .7091000000

H $9.8959790000-0.16324200004 .3648200000$

H 10.33438300000 .16928000002 .6876840000

C 11.81897900000 .73908100004 .0981200000

C 12.31704300000 .18075700005 .2698390000

H $11.6338930000-0.34562100005 .9284740000$

C 13.66809900000 .24780800005 .5796850000

H $14.0450680000-0.20429300006 .4882950000$

C 14.53256600000 .88357200004 .6974000000

H 15.59430600000 .93171000004 .9175960000

C 14.06042800001 .48717800003 .5411550000

C 12.68690900001 .44240300003 .2327710000

C 14.96415600002 .24593300002 .6161410000

H 15.05153300003 .28276600002 .9730090000

H 15.98175200001 .85054800002 .6524780000

C 14.43936500002 .24891600001 .2108000000

C 13.04437900002 .17534600001 .0292540000

C 15.27456100002 .36898000000 .1095300000

H 16.34701300002 .42194900000 .2681140000

C $14.75843800002 .4432060000-1.1786000000$

H $15.41940500002 .5482230000-2.0295510000$

C $13.38459800002 .3674250000-1.3623030000$

H $12.96784500002 .3988100000-2.3636440000$

C $12.52245500002 .2112110000-0.2831320000$

C $11.05353400002 .0497950000-0.4916650000$

H 10.68248600001 .16246200000 .0308150000

H $10.82829700001 .9331270000-1.5518680000$

C 9.48281600002 .62741000005 .4686910000

H 10.55466800002 .53942600005 .6820190000

C 9.08130700004 .07133700005 .7278430000

H 9.71203200004 .77815200005 .1916320000

H 9.1785500000 4.2912740000 6.7926000000

H 8.04408700004 .26752000005 .4538800000 
C 8.73643700001 .68250800006 .3984720000

H 7.65659700001 .81922700006 .3225590000 H 9.01280800001 .89038600007 .4334810000 H 8.95505200000 .63172200006 .2090240000 C 7.69886600001 .53533000003 .3758900000 H 7.4040780000 1.0349590000 4.3021700000 C 6.75864700002 .70927200003 .1532040000 H 6.73517200003 .37830700004 .0132420000 H 5.74073100002 .35308100002 .9867060000 H 7.0448980000 3.30580100002 .2864140000 C 7.64315900000 .49597500002 .2622960000 H 8.21646000000 .78254600001 .3796890000 H 6.61057600000 .34291300001 .9446520000 H $8.0288810000-0.46668400002 .5940950000$ C $8.32222200003 .1239700000-0.2893180000$ H 7.96959900002 .52667600000 .5517280000 C $7.47757800004 .3893700000-0.3233290000$ H 7.6042320000 5.00506300000 .5665250000 H $6.42174100004 .1199020000-0.3849640000$ H 7.7033710000 5.0060760000 -1.1928090000 C $8.13149000002 .2876150000-1.5459810000$ H $8.50901700002 .7805150000-2.4408230000$ H 7.0648010000 2.1126710000 -1.6991710000 H $8.60969100001 .3121170000-1.4707490000$ C $10.71739000004 .8684160000-0.8367160000$ H $11.79805900004 .7405240000-0.6974380000$ C $10.42430100004 .7480850000-2.3252630000$ H $10.68429900003 .7719660000-2.7327320000$ H $11.00791300005 .4918160000-2.8709350000$ H $9.37517200004 .9385340000-2.5508220000$ C $10.36096600006 .2521550000-0.3162110000$ H $9.29211000006 .4567640000-0.3661560000$ H $10.86135700007 .0074310000-0.9248170000$ H 10.68440500006 .39916200000 .7134110000 C 9.46382400005 .01435000002 .6101840000 C 12.51557300005 .03551700003 .1798300000 H 11.88882100004 .70079600001 .7638780000 H 13.50459700004 .83818900002 .7434660000 O 12.23929900006 .36620700003 .3176690000 H 12.73915400006 .86538500002 .6569080000

$\mathbf{T S}_{11,12}$

O 1.120612000010 .99954800004 .8676790000 
H 1.09626000009 .75246900005 .0054370000

H 1.11811100008 .75695400005 .1125630000

P - 2.24425100007 .88208800002 .8912920000

P 0.49461300006 .22083900004 .8077400000

O - 1.96840000008 .25659700006 .8366700000

N 0.84844500008 .24836500002 .4075780000

C - 1.63567600008 .01597700001 .1646940000

H - 1.07682700007 .09021100000 .9943740000

H - 2.49002400008 .01796100000 .4873480000

C - 0.76544300009 .19843600000 .9106450000

C - 1.174844000010 .22673500000 .0716370000

H - $2.166197000010 .1808140000-0.3678040000$

C $-0.324339000011 .2811570000-0.2344070000$

H - $0.650055000012 .0737850000-0.8960120000$

C 0.958610000011 .29159100000 .2984130000

H 1.641418000012 .09816300000 .0503330000

C 1.385092000010 .29645900001 .1674000000

C 0.51020500009 .25114300001 .5138190000

C 2.742207000010 .33305900001 .8056090000

H 3.477074000010 .77381300001 .1273020000

H 2.716919000011 .01164300002 .6700340000

C 3.18692900008 .97519600002 .2624020000

C 4.52899200008 .66417400002 .4291010000

H 5.27487200009 .40942600002 .1712190000

C 4.92532700007 .43451800002 .9397630000

H 5.97555400007 .20866700003 .0741120000

C 3.95746900006 .49667700003 .2720790000

H 4.25042800005 .52662600003 .6599390000

C 2.60618800006 .76412200003 .0869480000

C 2.20267700008 .02248600002 .5892330000

C 1.56997500005 .73673900003 .3966870000

H 2.0368070000 4.7756610000 3.6124710000

H 0.88879600005 .59848700002 .5509770000

C -3.0912850000 6.23922700002 .9008320000

H - 2.22217200005 .57294700002 .8651610000

C - 3.95205300005 .87652300001 .6985200000

H -4.89773900006 .41269700001 .6846660000$

H - 4.18855900004 .81181000001 .7453570000

H -3.4480570000 6.0499840000 0.7492810000

C -3.83437800006 .00978100004 .2079700000$

H -3.2467670000 6.2998060000 5.0803440000

H -4.0955190000 4.95580400004 .3196980000

H -4.7646560000 6.57893300004 .2312960000

C -3.4923270000 9.2537330000 2.9658470000 
H -2.9116060000 10.08414100002 .5489840000

C -3.9016950000 9.64069300004 .3780790000

H - 4.36959700008 .81387300004 .9139000000

H -4.6307680000 10.45205300004 .3377950000

H -3.0579720000 9.99173700004 .9678090000

C -4.71650800009 .05222400002 .0855920000$

H -4.4715010000 8.73065100001 .0738600000

H -5.2601360000 9.99515800002 .0028920000

H -5.4039550000 8.32409800002 .5163830000

C 1.72233000006 .30712200006 .2002010000

H 2.47427800006 .97227700005 .7581740000

C 1.21473400006 .97976800007 .4660860000

H 0.84485500007 .98582600007 .2778560000

H 2.03408100007 .06387300008 .1821230000

H 0.42163400006 .41254600007 .9514650000

C 2.40332000004 .98463900006 .5222330000

H 1.73412600004 .29934600007 .0423350000

H 3.25081000005 .16800400007 .1852210000

H 2.78835500004 .47586200005 .6396010000

C - 0.61263600004 .75861200005 .0935870000

H -1.4352360000 4.95141200004 .4062280000

C - 1.19825800004 .73045900006 .4979280000

H -0.4580690000 4.4296270000 7.2392020000

H -2.0091970000 4.00114500006 .5386520000

H -1.6096220000 5.69138900006 .8044790000

C - 0.02509800003 .40776500004 .7081030000

H 0.25719800003 .36330300003 .6576150000

H -0.77867500002 .63506900004 .8715050000$

H 0.84538300003 .13930700005 .3039440000

C 0.066967000011 .28663500004 .2207390000

H -0.081002000012 .35410300003 .9998530000$

C - 1.35366400008 .27207800005 .8453620000

$\mathrm{Ru}-0.37554900008 .28894100004 .3123320000$

O -0.7962030000 10.48107200003 .8264060000

$\mathbf{T S}_{13,14}$

Ru 8.754181000012 .337499000013 .1169950000

P 7.692568000014 .258443000012 .0652810000

P 8.464375000010 .499645000011 .7981530000

O 9.978553000013 .406544000014 .8329410000

O 9.941383000011 .422862000015 .9823570000

O 6.234594000011 .720139000014 .6210770000

N 10.547752000012 .817850000011 .9163170000 
C 8.893263000014 .853050000010 .8040820000

H 8.968462000014 .047852000010 .0666650000 H 8.497140000015 .733921000010 .2986890000

C 10.225470000015 .158930000011 .4060280000

C 10.702450000016 .465906000011 .4180970000 H 10.084761000017 .247853000010 .9893700000 C 11.953423000016 .772625000011 .9315600000 H 12.316232000017 .792464000011 .9164880000 C 12.736393000015 .753249000012 .4584870000 H 13.716134000015 .976434000012 .8682100000 C 12.277824000014 .445476000012 .4939780000 C 11.012270000014 .125691000011 .9608830000 C 9.896288000010 .430309000010 .6555330000 H 9.8388290000 9.4966170000 10.0929000000 H 9.740497000011 .25506800009 .9559200000 C 11.216147000010 .585821000011 .3268500000 C 12.15530200009 .562686000011 .3433780000 H 11.90001900008 .609563000010 .8917120000 C 13.41566700009 .760880000011 .8919780000 H 14.14561400008 .961427000011 .8870780000 C 13.732720000010 .998725000012 .4367320000 H 14.715107000011 .167278000012 .8658570000 C 12.800568000012 .026724000012 .4696520000 C 11.522867000011 .827706000011 .9215960000 C 13.067924000013 .342939000013 .1332140000 H 14.134641000013 .575042000013 .1358380000 H 12.784456000013 .269759000014 .1937110000 C 6.031458000014 .228390000011 .2411840000 H 6.006210000013 .240495000010 .7798370000 C 5.844355000015 .247490000010 .1276500000 H 4.846624000015 .13035000009 .7008010000 H 6.559385000015 .10821700009 .3178920000 H 5.927463000016 .274988000010 .4792710000 C 4.896156000014 .277840000012 .2532700000 H 4.7831170000 15.271082000012 .6868970000 H 5.0296930000 13.567859000013 .0693890000 H 3.956044000014 .032130000011 .7568850000 C 7.7371200000 15.6283160000 13.3179440000 H 8.817095000015 .703149000013 .4927400000 C 7.0959120000 15.2797030000 14.6532480000 H 7.2848410000 16.085048000015 .3652910000 H 7.5082420000 14.366555000015 .0805380000 H 6.0157570000 15.160995000014 .5822790000 C 7.2575120000 16.975000000012 .7982140000 
H 6.174991000016 .995734000012 .6707850000

H 7.7136780000 17.2469190000 11.8467870000

H 7.5125570000 17.756704000013 .5163540000

C 8.63011300008 .905656000012 .7309400000

H 9.70476700008 .913273000012 .9538280000

C 7.88748000008 .845105000014 .0546870000

H 6.80713700008 .914587000013 .9248180000

H 8.2010440000 9.6303820000 14.7402810000

H 8.0929470000 7.8898170000 14.5400320000

C 8.32841700007 .681905000011 .8790930000

H 8.66485200006 .785055000012 .4008040000

H 8.82892400007 .700711000010 .9111700000

H 7.2574750000 7.5698630000 11.7047510000

C 7.0171870000 10.3142020000 10.6703580000

H 7.0886930000 9.2856010000 10.3065880000

C 5.704055000010 .461047000011 .4208240000

H 5.587874000011 .457552000011 .8475040000

H 5.61691800009 .743239000012 .2365780000

H 4.865297000010 .294510000010 .7434860000

C 7.1329410000 11.24217400009 .4664090000

H 6.163979000011 .34578800008 .9760330000

H 7.8332580000 10.85571700008 .7280210000

H 7.4717670000 12.2439270000 9.7362000000

C 9.831485000012 .220121000015 .0964440000

H 9.7772580000 11.212378000013 .7594070000

C 7.2110780000 11.953665000014 .0363160000

$\mathrm{TS}_{15,10}$

O -0.1092920000 10.88655800003 .8090260000

P -2.0852960000 7.8565060000 3.0095680000

P 0.82068400006 .59609200004 .9412960000

O - 1.64691000008 .77765500006 .8552550000

N 0.94252900008 .35805200002 .3684540000

C - 1.53854300007 .63554800001 .2700400000

H - 0.91453700006 .73577200001 .2951580000

H - 2.40105100007 .42047600000 .6387420000

C -0.75825200008 .78653300000 .7214230000$

C $-1.19576000009 .4928590000-0.3940980000$

H - $2.14399300009 .2231840000-0.8464790000$

C $-0.419668000010 .4921100000-0.9644610000$

H - $0.771010000011 .0217430000-1.8408680000$

C $0.819196000010 .7915470000-0.4102910000$

H $1.437896000011 .5675900000-0.8491260000$

C 1.276754000010 .12049600000 .7127330000

C 0.49025400009 .10730400001 .2942770000 
C 2.580422000010 .44564600001 .3789220000

H 3.265737000010 .94044300000 .6884940000 H 2.3950580000 11.17294300002 .1833030000 C 3.19749700009 .21601100001 .9745500000 C 4.56734200009 .03093200002 .0859240000 H 5.23541600009 .78635000001 .6849930000 C 5.08733900007 .91013600002 .7235450000 H 6.15822000007 .78184300002 .8175370000 C 4.22070300006 .95158300003 .2314720000 H 4.61457100006 .06216700003 .7121740000 C 2.8435670000 7.0915060000 3.1020460000 C 2.31775400008 .24620700002 .4880520000 C 1.90392200006 .03157500003 .5699050000 H 2.45104800005 .14428200003 .8886370000 H 1.22176600005 .73484300002 .7668680000 C -3.0333290000 6.3106410000 3.3641970000 H - 2.26628900005 .55165600003 .1653720000 C - 4.21137100006 .03188800002 .4429120000 H -5.0474850000 6.6965480000 2.6583330000 H -4.5640180000 5.01181700002 .6053030000 H -3.9638470000 6.12525000001 .3866860000 C -3.4548260000 6.19697700004 .8227220000 H -2.6793420000 6.5249730000 5.5124410000 H -3.6953290000 5.1600600000 5.0635150000 H -4.3464490000 6.78904100005 .0244030000 C -3.2314930000 9.3158880000 2.9360050000 H - 2.515637000010 .14578200002 .8747200000 C - 4.05004400009 .48937700004 .2069070000 H - 4.86888400008 .77023200004 .2474750000 H - 4.497128000010 .48461100004 .2238830000 H -3.4646530000 9.3793000000 5.1182000000 C - 4.13376900009 .39570800001 .7128100000 H -3.5854030000 9.3409020000 0.7758880000 H -4.6607510000 10.35179800001 .7211860000 H - 4.89062600008 .61340400001 .7085820000 C 2.07388900006 .95254800006 .2677090000 H 2.76001500007 .60390700005 .7120190000 C 1.56221000007 .74974800007 .4568650000 H 1.14375300008 .70945600007 .1584230000 H 2.3930700000 7.9571280000 8.1333140000 H 0.80769700007 .21223200008 .0301410000 C 2.86102000005 .73243600006 .7265060000 H 2.25977300005 .07279300007 .3519770000 H 3.70974800006 .05943400007 .3297790000 
H 3.25956200005 .14491000005 .9008030000

C -0.17428000005 .10971400005 .4205010000$

H - 1.02472200005 .17217200004 .7383600000

C - 0.70224300005 .21124900006 .8445660000

H 0.08706000005 .03532600007 .5755300000

H - 1.46962100004 .45302600007 .0078020000

H -1.1485770000 6.1804500000 7.0654320000

C 0.49979500003 .76707800005 .1730540000

H 0.75081100003 .61584900004 .1247770000

H -0.1869430000 2.96833900005 .4589760000

H 1.40707400003 .63793000005 .7607940000

C - 0.830929000011 .70738800004 .6573910000

H - 1.075757000012 .63485400004 .1242730000

H - 1.771789000011 .24171600004 .9870170000

C - 1.09038100008 .66886900005 .8366330000

$\mathrm{Ru}-0.18312700008 .53105500004 .2573800000$

H 1.25387800009 .38610100004 .8897520000

H 0.761946000010 .13152000004 .5007740000

O -0.0920590000 12.11942600005 .7987570000

H - 0.117601000011 .40531500006 .4476070000

$\mathrm{TS}_{1,10}$

P -2.3070720000 7.8123510000 2.9385760000

P 0.38861800006 .09921200004 .9208950000

O - 2.35895100007 .78962600006 .8915490000

N 0.80261600008 .37456800002 .7423090000

C - 1.56833500008 .10473400001 .2836150000

H - 0.93395800007 .23120200001 .1099710000

H - 2.36167700008 .09191400000 .5338290000

C -0.73902200009 .33682200001 .1679700000$

C - 1.113114000010 .38068700000 .3322460000

H -2.0674050000 $10.3229190000-0.1817140000$

C -0.267253000011 .46047100000 .1115180000$

H - $0.564736000012 .2637290000-0.5506470000$

C 0.978878000011 .47794700000 .7246710000

H 1.663756000012 .29914900000 .5378830000

C 1.365603000010 .46874500001 .5963910000

C 0.48980200009 .39988900001 .8654610000

C 2.684522000010 .50233100002 .3112960000

H 3.444216000010 .99777200001 .7017470000

H 2.597830000011 .12850900003 .2122440000

C 3.13523900009 .13081800002 .7149720000

C 4.47661700008 .83352800002 .9077140000 
H 5.21564200009 .60558500002 .7177160000

C 4.87927200007 .58241500003 .3567220000

H 5.92818600007 .36528500003 .5138090000

C 3.91742100006 .60857700003 .5861160000

H 4.21535800005 .61646000003 .9091700000

C 2.56770600006 .86330600003 .3715490000

C 2.15252100008 .14898100002 .9548590000

C 1.55618900005 .77889500003 .5369320000

H 2.04935400004 .81847600003 .6865220000

H 0.92997800005 .69898200002 .6430900000

C -3.2112580000 6.2205900000 2.6670890000

H -3.9836010000 6.45634600001 .9308950000

C -3.9142550000 5.76245700003 .9386130000

H -3.2582920000 5.76148400004 .8101090000

H -4.3022960000 4.74970600003 .8163450000

H - 4.75983300006 .40716300004 .1771630000

C -2.2982810000 5.17065900002 .0347940000

H -2.2438090000 5.29788300000 .9546450000

H - 2.67706500004 .16541700002 .2273580000

H - 1.27394400005 .20991400002 .4068680000

C -3.63478200009.1123240000 3.0097150000

H -3.0830630000 10.00881200002 .7090270000

C - 4.18887200009 .35553500004 .4048580000

H -4.6760790000 8.47034300004 .8154750000

H -4.9397900000 10.14723100004 .3689290000

H -3.4151710000 9.6696950000 5.1010090000

C - 4.76194300008 .89249800002 .0117460000

H - 4.40725900008 .68107800001 .0030020000

H -5.3792520000 9.79074500001 .9552450000

H -5.4165830000 8.07458100002 .3163580000

C 1.54891200006 .12493300006 .3748560000

H 2.27371200006 .87405600006 .0337330000

C 0.94554300006 .64179300007 .6720630000

H 0.47091600007 .61371300007 .5485610000

H 1.73494500006 .75796200008 .4169590000

H 0.20951600005 .95729100008 .0912050000

C 2.30065100004 .82167100006 .6054550000

H 1.65427000004 .04567000007 .0153440000

H 3.09971600004 .98668500007 .3308080000

H 2.7631360000 4.42998700005 .7004710000

C - 0.65405600004 .56620400005 .0538020000

H - 1.48576500004 .79720700004 .3912650000

C - 1.24541800004 .36842900006 .4418660000

H - 0.50095300004 .02587400007 .1598590000 
H -2.0238480000 3.60438600006 .3967430000

H - 1.70301300005 .27418700006 .8380430000

C - 0.01067600003 .29027600004 .5317380000

H 0.27294900003 .36835500003 .4830790000

H - 0.72778600002 .47057600004 .6111810000

H 0.87338900003 .00155100005 .0987790000

C - 1.66382400007 .93037300005 .9621750000

$\mathrm{Ru}-0.57212400008 .15444800004 .5350520000$

O -1.1493610000 10.30291500004 .2697600000

H 0.686984000011 .19209600003 .9945390000

C - 0.223416000011 .15848300004 .6107560000

O 0.432909000010 .77494200005 .9810850000

H 0.80440500008 .60344100005 .5039040000

H 0.59537700009 .62359200005 .7774030000

H - 0.585401000012 .17830500004 .8069280000

H - 0.271085000010 .80289000006 .6505270000

\section{$\mathrm{H}_{2} \mathrm{O}$}

O -8.2271840000 -1.6389100000 6.2520390000

H -7.2656790000 - 1.58974500006 .2468220000

H - $8.5010740000-0.72105900006 .1546430000$

\section{EtSH}

C $-9.9558530000-0.09676000000 .0308470000$

C $-8.4496880000-0.0716910000-0.0235610000$

H - $10.39265200000 .3558640000-0.8596280000$

H - 10.33436500000 .44226000000 .8987270000

H - $10.3160800000-1.12517500000 .0889650000$

S -7.7348850000 $1.6032440000-0.0839600000$

H -8.0712110000 -0.6414900000 -0.8714220000

H -8.0148030000 - 0.51577500000 .8719590000

H -8.3215440000 $1.9804240000-1.2302140000$

\section{$\mathrm{MeOH}$}

C $-5.9167870000-0.7878820000-0.0046620000$

O $-4.4998440000-0.7990150000-0.0172300000$

H -6.3343220000 - 1.73998900000 .3358800000

H -6.3371480000 - $0.5568560000-0.9879490000$

H -6.2297140000 -0.0106690000 0.6906760000

H -4.2185740000 - $1.4904380000-0.6253810000$ 


\section{HCHO}

O -7.5825790000 -3.0691900000 -0.0036400000

C $-8.7844630000-3.0691900000-0.0036400000$

H $-9.3756140000-2.59714300000 .8060190000$

H $-9.3756140000-3.5412370000-0.8132990000$

\section{$\mathrm{HOCH}_{2} \mathrm{OH}$}

O -8.4385830000 -1.6737050000 6.0570180000

C $-7.0901240000-1.54888900006 .4289910000$

H $-8.8455630000-0.80030000006 .1144750000$

H -6.7193580000 -2.5700330000 6.5484220000

H -6.9844290000 -1.0086630000 7.3731880000

O -6.3278170000 -0.8313960000 5.4928590000

H -6.2574030000 -1.3673100000 4.6933440000

\section{$\mathrm{HCOOH}$}

C - $2.25684100001 .8748520000-1.4042650000$

O -2.6976030000 $1.8590950000-0.2827730000$

H - $2.64872900002 .4986220000-2.2196580000$

O - $1.23490900001 .1492920000-1.8409640000$

H $-0.89900800000 .6022550000-1.1098350000$

\section{Hydrogen}

H -5.8399560000 -0.7818910000 0.0000000000

H -5.0987590000 -0.8556050000 0.0000000000

$\mathrm{CO}_{2}$

C - $1.95618800001 .8306030000-1.1426770000$

O -1.0102470000 $1.8620690000-1.8108040000$

O -2.9021180000 $1.7991350000-0.4745340000$

CO

C -6.83160100001 .08559500000 .0000000000$

O -6.16996600000 .17368500000 .0000000000$ 


\section{Supporting References}

1. Kar, S.; Rauch, M.; Leitus, G.; Ben-David, Y.; Milstein, D. Highly Efficient Additive-Free Dehydrogenation of Neat Formic Ccid. Nat. Catal. 2021, 4, 193-201.

2. Rauch, M.; Luo, J.; Avram, L.; Ben-David, Y.; Milstein, D. Mechanistic Investigations of Ruthenium Catalyzed Dehydrogenative Thioester Synthesis and Thioester Hydrogenation. ACS Catal. 2021, 11, 2795-2807.

3. Heim, L. E.; Konnerth, H.; Prechtl, M. H. G. Future Perspectives for Formaldehyde: Pathways for Reductive Synthesis and Energy Storage. Green Chem. 2017, 19, 2347-2355.

4. Bone, W. A.; Smith, H. L. XCIV.-The Thermal Decomposition of Formaldehyde and Acetaldehyde. J. Chem. Soc. Trans. 1905, 87, 910-916.

5. Jenner, G.; Nahmed, E. M.; Libs-Konrath, S. Formaldehyde and Formates as Sources of Synthesis Gas via Ruthenium-Catalyzed Decomposition Reactions. J. Mol. Catal. 1991, 64, 337-347.

6. Nelson, W. L.; Engelder, C. J. The Thermal Decomposition of Formic Acid. J. Phys. Chem. 1926, 30, 470-475.

7. Xie, Y.; Ben-David, Y.; Shimon, L. J. W.; Milstein, D. Highly Efficient Process for Production of Biofuel from Ethanol Catalyzed by Ruthenium Pincer Complexes. J. Am. Chem. Soc. 2016, 138, 9077-9080.

8. Zhu, M.; Ge, Q.; Zhu, X. Catalytic Reduction of $\mathrm{CO}_{2}$ to $\mathrm{CO}$ via Reverse Water Gas Shift Reaction: Recent Advances in the Design of Active and Selective Supported Metal Catalysts. Trans. Tianjin Univ. 2020, 26, 172-187.

9. Ebrahimi, P.; Kumar, A.; Khraisheh, M. A Review of Recent Advances in Water-Gas Shift Catalysis for Hydrogen Production. Emergent mater. 2020 3, 881-917.

10. Zou, Y-Q.; von Wolff, N.; Anaby, A.; Xie, Y.; Milstein, D. Ethylene Glycol as an Efficient and Reversible Liquid Organic Hydrogen Carrier. 2019, Nat. Catal. 2, 415-422. 
11. Luo, J.; Rauch, M.; Avram, L.; Ben-David, Y.; Milstein, D. Formation of Thioesters by Dehydrogenative Coupling of Thiols and Alcohols with $\mathrm{H}_{2}$ Evolution. Nat. Catal. 2020, 3, 887-892.

12. Frisch, M. J. et al. Gaussian 16, Revision C.01; (Gaussian, Inc., Wallingford CT, 2016).

13. Zhao, Y.; Truhlar, D. G. A New Local Density Functional for Main-Group Thermochemistry, Transition Metal Bonding, Thermochemical Kinetics and Noncovalent Interactions. J. Chem. Phys. 2006, 125, 194101/1-18.

14. Weigend, F.; Ahlrichs, R. Balanced Basis Sets of Split Valence, Triple Zeta Valence and Quadruple Zeta Valence Quality for $\mathrm{H}$ to $\mathrm{Rn}$ : Design and Assessment of Accuracy. Phys. Chem. Chem. Phys. 2005, 7, 3297-3305.

15. Weigend, F. Accurate Coulomb-Fitting Basis Sets for H to Rn. Phys. Chem. Chem. Phys. 2006, 8, 1057-1065.

16. Grimme, S.; Antony, J.; Ehrlich, S.; Krieg, H. A Consistent and Accurate Ab Initio Parametrization of Density Functional Dispersion Correction (DFT-D) for the 94 Elements HPu. J. Chem. Phys. 2010, 132, 154104/1-19.

17. Neese, F. Software Update: The ORCA Program System, Version 4.0. WIREs Comput. Mol. Sci. 2018, 8, e1327-e1332.

18. Mardirossian, N.; Head-Gordon, M. $\omega B 97 X-V:$ A 10-Parameter, Range-Separated Hybrid, Generalized Gradient Approximation Density Functional with Nonlocal Correlation, Designed by a Survival-Of-The-Fittest Strategy. Phys. Chem. Chem. Phys. 2014, 16, 9904-9924.

19. Vydrova, O. A., and Voorhis, T. V. (2010). Nonlocal van der Waals density functional: The simpler the better. J. Chem. Phys. 2017, 133, 244103/1-9.

20. Hujo, W., and Grimme, S. (2011). Performance of the van der Waals Density Functional VV10 and (hybrid)GGA Variants for Thermochemistry and Noncovalent Interactions. J. Chem. Theory Comput. 2017, 7, 3866-3871.

21. Hellweg, A.; Hattig, C.; Hoefener, S.; Klopper, W. Optimized Accurate Auxiliary Basis Sets for RI-MP2 and RI-CC2 Calculations for the Atoms Rb to Rn. Theor. Chem. Acc. 2007, 117, 587-597. 
22. Iron, M. A.; Janes, T. Evaluating Transition Metal Barrier Heights with the Latest Density Functional Theory Exchange-Correlation Functionals: The MOBH35 Benchmark Database. J. Phys. Chem. A 2019, 123, 3761-3781.

23. Marenich, A. V.; Cramer, C. J.; Truhlar, D. G. Universal Solvation Model Based on Solute Electron Density and on a Continuum Model of the Solvent Defined by the Bulk Dielectric Constant and Atomic Surface Tensions. J. Phys. Chem. B 2009, 113, 6378-6396.

24. Cramer, C. J. Essentials of Computational Chemistry: Theories and Models in $2^{\text {nd }}$ Edition. (John Wiley, and Sons Ltd: West Sussex, England, 2014).

25. Sparta, M.; Riplinger, C.; Neese, F. Mechanism of Olefin Asymmetric Hydrogenation Catalyzed by Iridium Phosphino-Oxazoline: A Pair Natural Orbital Coupled Cluster Study. J. Chem. Theory and Computation 2014, 10, 1099-1108.

26. Hopmann, K. H. How Accurate is DFT for Iridium-Mediated Chemistry?. Organometallics 2016, 35, 3795-3807.

27. Gusev, D. G. Revised Mechanisms of the Catalytic Alcohol Dehydrogenation and Ester Reduction with the Milstein PNN Complex of Ruthenium. Organometallics 2020, 39, 258-270. 